\title{
Social choice: locating public facilities \& voting in a large electorate
}

Citation for published version (APA):

Chatterjee, S. (2017). Social choice: locating public facilities \& voting in a large electorate: two location problems and a voting problem. [Doctoral Thesis, Maastricht University]. Datawyse / Universitaire Pers Maastricht. https://doi.org/10.26481/dis.20170920sc

Document status and date:

Published: 01/01/2017

DOI:

10.26481/dis.20170920sc

Document Version:

Publisher's PDF, also known as Version of record

\section{Please check the document version of this publication:}

- A submitted manuscript is the version of the article upon submission and before peer-review. There can be important differences between the submitted version and the official published version of record.

People interested in the research are advised to contact the author for the final version of the publication, or visit the DOI to the publisher's website.

- The final author version and the galley proof are versions of the publication after peer review.

- The final published version features the final layout of the paper including the volume, issue and page numbers.

Link to publication

\footnotetext{
General rights rights.

- You may freely distribute the URL identifying the publication in the public portal. please follow below link for the End User Agreement:

www.umlib.nl/taverne-license

Take down policy

If you believe that this document breaches copyright please contact us at:

repository@maastrichtuniversity.nl

providing details and we will investigate your claim.
}

Copyright and moral rights for the publications made accessible in the public portal are retained by the authors and/or other copyright owners and it is a condition of accessing publications that users recognise and abide by the legal requirements associated with these

- Users may download and print one copy of any publication from the public portal for the purpose of private study or research.

- You may not further distribute the material or use it for any profit-making activity or commercial gain

If the publication is distributed under the terms of Article $25 \mathrm{fa}$ of the Dutch Copyright Act, indicated by the "Taverne" license above, 


\section{Social Choice: Locating Public Facilities \& Voting in a Large Electorate}

TWO LOCATION PROBLEMS AND A VOTING PROBLEM

NETHERLANDS, 2017

WRITTEN BY

SWARNENDU CHATTERJEE

Maastricht University

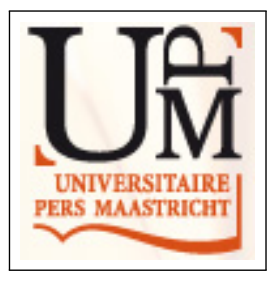

2017

THE PUBLISHER 



\title{
Social Choice: Locating Public Facilities \& Voting in a Large Electorate
}

TwO LOCATION PROBLEMS AND A VOTING PROBLEM

\author{
DISSERTATION
}

\begin{abstract}
TO OBTAIN THE DEGREE OF DOCTOR AT THE MAASTRICHT UNIVERSITY, ON THE AUTHORITY OF THE RECTOR MAGNIFICUS,

PROF.DR. RIANNE M. LETSCHERT

IN ACCORDANCE WITH THE DECISION OF THE BOARD OF DEANS, TO BE DEFENDED IN PUBLIC

ON WEDNESDAY $20^{\text {th }}$ SEPTEMBER 2017, AT 10:00 HOURS
\end{abstract}

BY

SWARNENDU CHATTERJEE 


\section{Supervisor}

Prof. dr. H.J.M. Peters

\section{Co-supervisor}

Dr. A.J.A. Storcken

\section{Assessment Committee}

Prof. dr. Dries Vermeulen (Chairman)

Dr. Burak Can

Prof. dr. A.M.A. van Deemen (Radboud University, Nijmegen)

Dr. Ulle Endriss (University of Amsterdam, Amsterdam)

Dit onderzoek werd financieel mogelijk gemaakt door de Graduate School of Business and Economics (GSBE). 
To my parents and fiancée. 



\section{Acknowledgements}

My years as a PhD student at Maastricht University have been one of the most significant and interesting periods in my life. I truly enjoyed every single moment of it. This thesis would not have been possible without the enormous help and support of many people whom I would like to acknowledge here.

First and foremost, I would like to express my deepest gratitude to my supervisor, Hans Peters. Throughout the years of my stay in Maastricht, he has been a real friend and a great teacher to me. I thank Hans for his insightful comments on my research. His weekly group meetings have always been a stimulating experience and a source of new ideas for me. I benefited from his excellent guidance during my $\mathrm{PhD}$. I especially thank him for his great support during the last year of my $\mathrm{PhD}$.

Furthermore, I am heartily thankful to my co-supervisor Ton Storcken whose guidance and advice enhanced greatly the quality of this thesis. He motivated me whenever I struggled with problems. I like the way he explains difficult concepts with simple stories.

I also would like to thank the members of my assessment committee Dries Vermeulen, Burak Can, A.M.A. van Deemen, Ulle Endriss for taking the time to read and referee the manuscript.

I extend my gratitude to all my colleagues and friends at the Department of Quantitative Economics for providing a nice and enjoyable working atmosphere. Especially, I 
would like to thank the two lovely secretaries of the department, Karin van den Boorn and Yolanda Paulissen for their great help.

Finally, I owe my gratitude to professor Arunava Sen who was my inspiration for pursuing a $\mathrm{PhD}$. Last but not the least, I would like to express my love and gratitude to my parents and fiancée for their love and their support during these years. This thesis is dedicated to them.

Swarnendu Chatterjee

Maastricht, July 2017 
Contents

Contents

List of Figures $\quad$ vii

List of Tables $\quad$ ix

1 Introduction 1

$1.1 \quad$ Locating a Public Good on a Sphere . . . . . . . . . . . . . . . . . 4

$1.2 \quad$ Locating Two Public Goods in $\mathbf{R}^{2} \ldots \ldots \ldots$. . . . . . . . . . 4

1.3 Frequency Based Analysis of Voting Rules . . . . . . . . . . . . . . 5

\begin{tabular}{ll}
\hline Location Problems & 7
\end{tabular}

2 Locating a Public Good on a Sphere 9 


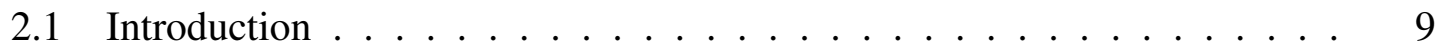

2.2 Model and Preliminaries .................... 10

$2.2 .1 \quad$ Some Facts about Spherical Geometry . . . . . . . . . . . . . . . 11

2.3 Results . . . . . . . . . . . . . . . . . . . . . . . . . . 12

2.4 Strict Strategy-proofness and Coalitional

Strategy-proofness . . . . . . . . . . . . . . . . 16

$3 \quad$ Locating Two Public Goods in $\mathbb{R}^{2} \quad 23$

B. Introduction . . . . . . . . . . . . . . . . . 23

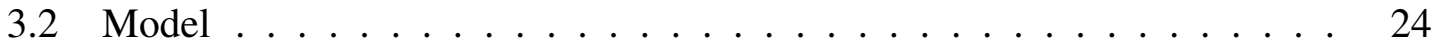

3.3 Implications of the Properties . . . . . . . . . . . . . . . . . . . . . 29

$3.4 \quad$ Double Generalised Median Rule and Its Properties . . . . . . . . . . . . 39

3.4.1 Properties of Double Generalised Median Rule . . . . . . . . . . 40

B.5 Characterisation Result . . . . . . . . . . . . . . . . . . 43

B.6 Interaction of the Conditions . . . . . . . . . . . . . . . . 47

II Voting Problem

4 Frequency Based Analysis of Voting Rules 53

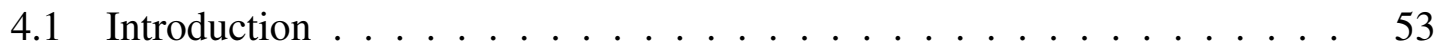

4.2 Model . . . . . . . . . . . . . . . . . . . . 56

$4.2 .1 \quad$ Unimodal Frequency Distributions . . . . . . . . . . . 57

4.2 .2 Detinitions of Some Conditions . . . . . . . . . . . . 59

4.3 Decisions in Unimodal Frequency Distributions . . . . . . . . . . . . 61 
4.4 Sufficient Conditions for Choosing the Mode . . . . . . . . . . . . . . . 64

4.5 Robustness of the Mode-selection Property $\ldots \ldots$. . . . . . . . . 68

4.6 Multimodal Frequency Distributions . . . . . . . . . . . . . . . . . . . 79

47 Conclusion . . . . . . . . . . . . . . . . . . . 82

4.8 Appendix-1 . . . . . . . . . . . . . . . . . 83

4.9 Appendix-2 $\ldots \ldots \ldots \ldots$. . . . . . . . . . . . . . . . . 88

Bibliography ............................... 89

$\begin{array}{ll}\text { Bibliography } & 91\end{array}$

$\begin{array}{ll}\text { Valorisation } & 95\end{array}$

\begin{tabular}{ll}
\hline Curriculum Vitae & 97
\end{tabular}

$\begin{array}{lr}\text { Index } & 97\end{array}$ 

List of Figures

2.1 Lemma 2.3 $\ldots \ldots \ldots \ldots \ldots$

2.2 Second part of the proof of Proposition 2.1 . . . . . . . . . . . . . 17

2.3 Spherical Coordinate System . . . . . . . . . . . . . . . . . . . . 19

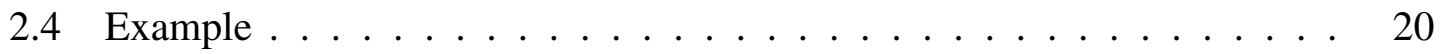

3.1 Quadrants with center at $t \in \mathbf{R}^{2} \ldots \ldots \ldots 25$

3.2 Partition of $\mathbf{R}^{2}$ based on $F^{A}$ and $F^{B} \ldots \ldots \ldots 27$

3.3 Suitable Elliptical Indifference Curve . . . . . . . . . . . . . . 30

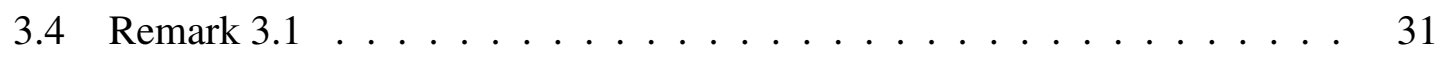

B.5 Proof ofLemma $3.4 \ldots \ldots \ldots$

3.6 Partition of $\mathbf{R}^{2}$ based on $F^{A}$ and $F^{B} \ldots \ldots . \ldots 34$

3.7 Proof ofLemma $3.7 \ldots \ldots \ldots \ldots$

vii 
B.8 Proof ofLemma 3.7 . . . . . . . . . . . . . . . 36

B.9 Proof ofLemma $3.7 \ldots \ldots \ldots$

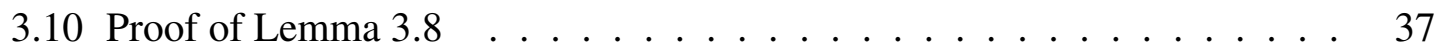

B.川 Proof ofLemma 3.9 . . . . . . . . . . . . . . . 38

B.12 Proof oflemma $3.9 \ldots \ldots \ldots$

B.13 Proof ofLemma 3.9 . . . . . . . . . . . . . . . . 38

3.14 Description of the rule $\ldots \ldots \ldots$. . . . . . . . . . . . 47

3.15 Deviation example . . . . . . . . . . . . . . . . . . . . 48

4.1 Symmetric unimodal distribution with 4 candidates $\ldots \ldots$. . . . . . 58

4.2 Symmetric unimodal distribution with 4 candidates $\ldots . . . . . . . .59$

4.3 Tail-perturbed unimodal distribution . . . . . . . . . . . . . . . . 70

4.4 Robustness of Condorcet consistent rules . . . . . . . . . . . . . . . . . 72

4.5 Robustness of Borda rule . . . . . . . . . . . . . . . . . . . . 75

4.6 Robustness of Plurality rule $\ldots \ldots \ldots \ldots$

4.7 Multimodal distribution f.................. 81 
List of Tables

$4.1 \quad$ Frequency distribution with three alternatives $\ldots \ldots \ldots 78$

4.2 Pairwise comparison $\ldots \ldots \ldots \ldots$. . . . . . . . . . . . 79

4.3 Multimodal distribution f . . . . . . . . . . . . . . 80

4.4 Occurrence of a Cycle $\ldots \ldots \ldots \ldots$

4.5 Non-occurrence of a Cycle . . . . . . . . . . . . . . . . 89 



\section{CHAPTER 1}

\section{Introduction}

This thesis contributes to the theory of social choice. As the name suggests, social choice theory deals with choices of society. A choice of society may concern a great variety of things, for instance, choosing a location to place a public facility, choosing candidates in an election etc. Now, being rational every individual in society wants the collective choice to be optimal for herself. Obviously, they express different opinions about the choice to be made. This creates a problem of aggregating these different opinions or preferences into a single social choice. The underlying assumption is that individual preferences are not known but everybody knows the available choices. A social choice function models this aggregation of individual preferences to a collective choice. A social choice function should have "good" properties. Being good means different things in different contexts. One of the most important properties is that no individual should be able to manipulate the collective choice, making it favourable to her. This essentially makes sure that every individual is "honestly" reporting her preferences. Another desirable property is that it takes into account all individual preferences. Of course there are several other properties considered to be desirable. The most important question is whether it is possible to design a social choice function that is reasonable in the sense that it satisfies some of the properties. 
In this regard, [Arrow, 1950], [Gibbard, 1973], [Satterthwaite, 1975] have shown that it is generally impossible to find a social choice function satisfying all these desired criteria. These results are called "impossibility results/theorems" in literature. However, there are some remarks concerning the use of these results. Arrow has said, "Most systems are not going to work badly all of the time. All I proved is that all can work badly at times." In his general impossibility result individuals are allowed to have any conceivable preferences. There is a stream of literature showing that it is possible to escape from "impossibility" if we do not assume the individuals to have all conceivable preferences. Specifically, the possibility of having some positive results depends mostly on two things, namely, the set of admissible preferences and properties of the social choice function.

Two major ways of restricting the set of admissible preferences are studied in this thesis. One way is to restrict the set of preferences for every individual such that all of them have the same set of admissible preferences from which they can choose independently. Another way is to restrict the preference combinations of society as a whole, rather than imposing restrictions over each individual. In this thesis we study both these kinds of restrictions. The former is studied in Part I and the latter is studied in Part IIl. A particular type of preference called single-peaked preference is well studied in literature and expected to induce possibilities of a collective decision in most situations. Single-peaked preferences mean that every individual has a best point (peak) that she wants to be chosen and her dislike for any other choice increases with the distance of the choice from her peak. In Part II of this thesis we work with this type of preferences in two different problems. The choice of distance function depends on the context of the social choice problem. In Chapter $\square$ we have taken Spherical distance as the suitable distance function, whereas in Chapter B] we have taken Euclidean distance. Similarly, we can have preferences which are single-dipped, meaning that for every individual there is a worst point (dip), that she dislikes the most and her preference for any other choice increases with the distance of the choice from the dip. In Chapter $\square$ we deal with a choice problem where having a single-peaked preference is equivalent to having a single-dipped preference. Chapter 4 deals with voting problems in a very general context, where we put restrictions on the set of conceivable preferences of all the voters collectively. More specifically, there we treat preference profiles as frequency distributions: Restrictions on profiles are described by frequency distributions. In large electorates this approach seems to be natural.

As mentioned above there can be several properties of a social choice function. We impose some properties which of course may vary across the chapters. To give a general idea of the properties that are used in this thesis, we briefly describe them below.

In literature, non-manipulability is a very well studied notion which ensures that no in- 
dividual can misreport her preference such that the resulting choice becomes better for her. This non-manipulability can be applied with varied strength, for instance, a social choice function could be immune to misreport by a group of individuals than by just a single agent. Obviously, imposing stronger conditions on the social choice function does not increase the set of possible choices, potentially increasing the chance of having an impossibility. On the other hand, the non-manipulability condition is considered to be desirable for a social choice function. Since, under non-manipulability truth telling is the dominant strategy, individuals do not need to take care about others' strategies. As a result, essentially individuals do not need think of any strategies, they can just report their honest preferences. Thus, a non-manipulable social choice function reduces the requirement of individual intelligence or computing abilities. In turn, it protects unsophisticated individuals from strategic errors. Such a social choice function also reduces the effort from the authority needed to reveal true individual preferences. The resulting outcome is not sensitive on each individual beliefs about other individual preferences. As a result the final choice is based on the correct information. In the words of [ Vickrey, 1961], in second-price auctions: "Each bidder can confine his efforts and attention to an appraisal of the value the article would have in his own hands, at a considerable saving in mental strain and possibly in out-of-pocket expense.

Pareto optimality is another well-studied notion which takes into consideration everyone's utility from the choice and compares it to other available choices. In other words, it ensures that the social choice function provides the best collective choice in the sense that any other choice makes at least one individual worse-off.

Uncompromisingness makes a social choice function immune to extreme individual reports. Due to uncompromisingness a social choice function remains constant over certain sets of preferences. These sets are determined based on the choice problem under consideration.

Part II of the thesis elaborates on two location problems, namely, Locating a public good on a sphere and Locating two public goods in $\mathbf{R}^{\mathbf{2}}$. Part $\mathbf{l l}$ analyses several Voting methods.

Now, we give a short description of the particular areas of social choice we are dealing with in the subsequent chapters. 


\section{Locating a Public Good on a Sphere}

In chapter $\square$ the concern is how to locate a public good/bad on a sphere (the earth). This chapter is based on [Chatterjee et al, 2016]. Here we have a finite set of agents and the set of alternatives, i.e., the set of possible locations for the public good is the unit sphere. We assume that everyone wants the public good to be located at her most preferred point on the sphere called the peak of her preference. For all other points the preference decreases with distance from the peak. Note that, on a sphere one's least preferred point is the diametrically opposite point of one's peak. Hence a single-peaked preference can be reformulated as a single-dipped preference. So, results based on single-peaked preferences translate to similar results on single-dipped preferences. Therefore, this research applies to locating global public goods for example global internet servers, storage for all human knowledge etc. Also this work applies to locating global public obnoxious facilities like nuclear power plants, scientific research projects involving nuclear reactions etc. Collective decision making is modelled by a social choice function or rule that takes agents' preferences as inputs and its output is the location of the public good. We impose two desirable conditions on the social choice function. These conditions are: non-manipulability and Pareto optimality. Specifically, we investigate two different types of non-manipulability, namely, "coalitional strategy-proofness", where no group of agents can manipulate in its favour, and "strict strategy proofness" where any individual deviation leads to a strictly worse outcome for that individual. We end up with an "impossibility" result, which means that there is no non-dictatorial social choice rule that satisfies the conditions we imposed. This result stands out in the stream of literature because usually having single-peaked preferences helps achieving possibility results. It is still an open problem whether mere strategy-proofness and Pareto optimality imply dictatorship.

\section{Locating Two Public Goods in $\mathbf{R}^{2}$}

In chapter B, we discuss the location of multiple public goods. Suppose we have to locate two libraries in a town. It is natural to assume that agents prefer to have at least one library close to their homes or some preferred place and preferences for all other locations decrease with the distance from that most preferred locations. Therefore, preference for a single library can be modelled as a single-peaked preference in the two dimensional Euclidean space. As we are deciding on the location of two libraries together, we need to have joint preferences over pairs of locations. These joint preferences are obtained by 
combining the marginal preferences for a single library. There can be several different types of joint preferences, we use the so called lexmax extension. Lexmax extension means that, an agent prefers a pair of locations $\left(L_{1}, L_{2}\right)$ to another pair of locations $\left(L_{1}^{\prime}, L_{2}^{\prime}\right)$ if the nearest location out of these four locations is from the first pair and in case the nearest ones are in both the pairs but the second nearest location is from the first pair. Thus first we order two locations within a pair in decreasing order of their utility and then between pairs comparison is made lexicographically. In the one dimensional case several results are known ([see Miyagawa, 2001], [see Ehlers, 2001], [see Ehlers, 2002], [see Heo, 2013], [see Gordon, 2007]). Here we mainly study this location problem assuming the social choice function to be "non-manipulable" and "uncompromising". Under these conditions we have been able to characterise the class of rules. Specifically, for each location we apply a generalised median voter rule per coordinate.

\section{Frequency Based Analysis of Voting Rules}

In the final chapter 4 , the issue is on anonymous collective decision making in large electorates. This chapter is based on [Chatterjee and Storcken, 2017]. We study frequency distributions over the set of preferences based on the structure on linear orderings induced by the Kemeny distance. We observe that country wide elections deal with large electorates where preferences over the candidates may be quite diverse. Cultural backgrounds, media or social networks may influence the voters and bring in coherency among some of these voters preferences. In this chapter we study consequences of this coherence by modelling voters preference combinations, also known as (preference) profiles, by frequency distributions. In particular we look at the case where coherency is concentrated around one mode. So, the distribution is unimodal. Here we did not take normative conditions as the primary starting point nor did we impose restrictions on the admissible voters' preferences. But we rather considered whether the given distribution could give rise to a "natural/appealing" collective decision. For instance in the hypothetical case that all voters' preferences are equal to each other this preference is a natural collective decision. Or in case of three candidates and voters' preferences are distributed evenly among the three preferences of a Condorcet profile we may assume that the outcome is complete indifference. Of course these situations have only hypothetical value and also this approach leads more than often to ad hoc solutions. Strictly speaking, however, a collection of normative conditions also defines classes of profiles at which the collective decision is structured. More importantly, at the unimodal preference distributions considered here many wellknown collective decision rules have the mode as outcome. We therefore consider it to 
be a natural and appealing collective decision at such distributions. To be more explicit Condorcet consistent rules, score rules and various elimination rules all have the mode as the outcome. For many rules this follows because the mode embodies the "positional" order, the pairwise comparison order as well as the order of "elimination".

Further, we discuss a set of sufficient conditions for a rule such that it assigns the mode at a unimodal distribution. Besides neutrality and anonymity this set contains a monotonicity condition and a condition called discrimination. In general monotonicity properties are usually restrictive and therefore not satisfied by reasonable collective decision rules. The one discussed here is satisfied by many rules, for instance pairwise rules and score rules. Discrimination means that a rule cannot be indifferent between two candidates, say $a$ and $b$; whenever the frequency of every preference $R$ at which $a$ is preferred to $b$ outnumbers frequency of $R^{\prime}$, where $R^{\prime}$ is the preference obtained from $R$ by swapping the position of $a$ and $b$ in $R$.

We tried to find out how sensitive choosing the mode is at several decision rules when small perturbations in the tail of the distribution are possible. For Condorcet consistent rules, Borda rule and Plurality rule we show that all three assign the mode at the following type of perturbed unimodal distribution. Frequency declines with the distance to the mode until about half the maximal distance. Beyond this point frequencies are free but constant at each specific distance from the mode and they are bounded above by the frequency at half the maximal distance. The combinatorial reasoning by which these results are deduced might most probably work similarly for other collective decision rules such as other score rules or some elimination rules. But we have not found a general way to deduce this for instance for all score rules. The results found here indicate, however, that the mode choice of Condorcet consistent, Borda and Plurality rules is robust to considerable perturbations in the tail part of a single mode distribution.

Our analysis includes multimodal distributions resulting from superposing two or more unimodal distributions. Indeed one may hope that any distribution can be thought of as the union of several unimodal distributions. Then the collection of outcomes at each of these may somehow determine the outcome of the original distribution. For discriminating collective decision rules we show that the outcome at the union of two unimodal distributions is between the two modes of these unimodal distributions. We do not have conclusive results for multimodal distributions except some basic ones.

The Chapters are intended to be self-contained. We also try to unify the symbols across the chapters. 


\section{Part I}

\section{Location Problems}



CHAPTER 2

\section{Locating a Public Good on a Sphere}

\section{Introduction}

It is well known that restricting preferences to be single-peaked may be a means to escape the [Gibbard, 1973] and [Satterthwaite, 1975] theorems, which say that strategy-proof social choice functions on the full preference domain and with range cardinality at least three, are dictatorial. Single-peaked preferences were studied already in [Black, 1948]. In his "Single-peakedness theorem" [Black, 1948] noted: If all candidates are points on a line, and each voter has a "singlepeaked" utility function then a Condorcet cycle (see section (4.9) is impossible, hence with honest rank-order votes there is a transitive social ordering and hence a Condorcet winner. In this context [Moulin, 1980] has shown that strategic considerations justify the central role given to the Condorcet procedure which amounts to elect the 'median' peak: namely all strategy-proof anonymous and efficient voting schemes can be derived from the Condorcet procedure by simply adding some fixed ballots to the agents' ballots (with the only restriction that the number of fixed ballots is strictly less than the number of agents). [Border and Jordan, 1983] have shown that with single peaked preferences in multidimension, if a non-manipulable choice function satisfies a weak unanmity-respecting condition then a choice function will decompose into 
a product of one-dimensional mechanisms in the sense that each coordinate of the chosen point depends only on the respective coordinate of the voters' ideal points. [Kim and Roush, 1981] provide an alternative axiomatic characterization of the same class of solutions. Then [Peters et al, 1992] have shown that in two dimensional location problems any Pareto optimal, anonymous, strategy-proof solution must be of the following form: Choose a pair of orthogonal axes in the plane. Coordinates of points are projections on these axes. From the reported points $x_{1}, \ldots, x_{n}$, determine the two median coordinates; these coordinates are the coordinates of the solution point. This list of reference is not exhaustive. In all those papers, typically, coordinatewise median-like rules turn out to be strategy-proof, besides satisfying other desirable properties. In the present chapter, agents have single-peaked preferences on the sphere, i.e., the surface of the three-dimensional unit ball. We show that every Pareto optimal rule that is strict strategy-proof or coalitional strategy-proof must be dictatorial - this in spite of the single-peaked preference assumption. Here, strict strategy-proofness means that if an agent misreports, either he is strictly worse off or the outcome assigned by the rule does not change at all. It is still an open problem whether mere strategy-proofness and Pareto optimality imply dictatorship: an example at the end of the chapter suggests that it does, but we do not have a proof.

We follow similar arguments as in ' Ozt'urk et al, 2013.'Ozt'urk et al, 2013.'Ozt'urk et al, 2013, who consider single-dipped preferences on the two-dimensional disc. Of course, on the sphere, every single-peaked preference is a single-dipped preference if we take the antipodal point of the peak as the dip - so the results in this chapter also hold for singledipped preferences.

\section{Model and Preliminaries}

The set of alternatives is the unit sphere $A=\left\{x \in \mathbb{R}^{3}:|x|=1\right\}$, where $|$.$| denotes Euclidian$ distance. The set of agents is $N=\{1, \ldots, n\}$ with $n \geq 2$. Subsets of $N$ are called coalitions. Each agent $i \in N$ has a single-peaked preference on $A$, denoted by its peak $p(i)$ : a point $x \in A$ is weakly preferred to a point $y \in A$ if $|x-p(i)| \leq|y-p(i)|$. Here, distance is measured along the sphere, but since this distance is isomorphic with Euclidian distance within the closed unit ball, we just keep the same notation $|$.$| .$

A profile is a vector $p=(p(1), p(2), \ldots, p(n)) \in A^{N}$. A social choice function or rule $\varphi$ assigns to each $p \in A^{N}$ a collective decision $\varphi(p) \in A$.

Definition 2.1. A point $x \in A$ is Pareto optimal for a profile $p \in A^{N}$ if there is no $x^{\prime} \in A \backslash\{x\}$ 
such that $\left|x^{\prime}-p(i)\right| \leq|x-p(i)|$ for all $i \in N$ with at least one strict inequality. Rule $\varphi$ is Pareto optimal if $\varphi(p)$ is Pareto optimal for every $p \in A^{N}$.

Definition 2.2. Let $S \subseteq N$. Profiles $p$ and $q$ are $S$-deviations if $p(i)=q(i)$ for all $i \in N \backslash S$.

Definition 2.3. Rule $\varphi$ is manipulable by $S \subseteq N$ at $p \in A^{N}$ via $q \in A^{N}$ if $p, q$ are $S$ deviations and $|\varphi(p)-p(i)| \geq|\varphi(q)-p(i)|$ for all $i \in S$, with at least one inequality strict. Therefore, $\varphi$ manipulable by $S \subseteq N$ if there are $S$-deviations $p, q \in A^{N}$ such that $\varphi$ is manipulable at $p$ via $q$

Definition 2.4. Rule $\varphi$ is

1. coalitional strategy-proof if it is not manipulable by any $S \subseteq N$;

2. intermediate strategy-proof if it is not manipulable by any $S$ at $p$ via $q$ such that $p(i)=p(j)$ for all $i, j \in S$;

3. strategy-proof if it is not manipulable by any $\{i\}, i \in N$;

4. strict strategy-proof if for all $i \in N$ and $\{i\}$-deviations $p$ and $q$, either $\varphi(p)=\varphi(q)$ or $|\varphi(p)-p(i)|<|\varphi(q)-p(i)| ;$ and

5. intermediate strict strategy-proof if for all $S \subseteq N$ and $S$-deviations $p, q \in A^{N}$ with $p(i)=p(j)$ for all $i, j \in S$, either $\varphi(p)=\varphi(q)$ or $|\varphi(p)-p(i)|<|\varphi(q)-p(i)|$ for all $i \in S$.

Definition 2.5. A coalition $S \subseteq N$ is decisive for $\varphi$ if for every $x \in A$ and every profile $p \in A^{N}$ with $p(i)=x$ for all $i \in S$ we have $\varphi(p)=x$.

If for a particular agent $d$ coalition $\{d\}$ is decisive, then agent $d$ is a dictator. In that case, $\varphi$ is dictatorial. We conclude this section with some spherical terminology, notations, and facts.

\section{Some Facts about Spherical Geometry}

- Each point $x \in A$ has an antipodal, i.e., the point of $A$ with maximal distance to $x$.

- A great circle $G$ is the intersection of $A$ with a plane through the origin. A great circle $G$ divides $A$ into two disjoint, open hemispheres. 
- We denote the great circle through any two points $x \in A$ and $y \in A$ by $G_{x y}$. If $x$ and $y$ are antipodal, then $G_{x y}=A$.

- For a great-circle $G$ and a point $t \in A \backslash G,(G, t)$ denotes the (open) hemisphere of all points of $A$ on the same side of $G$ as $t$, excluding $G$. The closed hemisphere is denoted by $\overline{(G, t)}$, hence $\overline{(G, t)}=(G, t) \cup G$.

- For distinct non-antipodal points $x, y \in A,[x \frown y]$ denotes the shortest closed arc between $x$ and $y$, which is a subset of the great circle through $x$ and $y$. For $x, y \in A$ $(x \neq \hat{y})$ we denote the closed (shortest) arc by $[x \frown y]$, clearly $[x \frown y] \subseteq G_{x y}$. Also, $[x \frown y[=[x \frown y] \backslash\{y\}], x \frown y]=[x \frown y] \backslash\{x\}], x \frown y[=[x \frown y] \backslash\{x, y\}$. For $x=$ $\hat{y} \in A$, there are infinitely many great-circles through them. If $x$ and $y$ are antipodal points then $[x \frown y]=A$. If $x=y$ then $[x \frown y]=\{x\}$.

- $\triangle a b c$ denotes the spherical triangle with sides $[a \frown b],[b \frown c],[c \frown a]$. The sum of the angles of a triangle varies from $180^{\circ}$ to $540^{\circ}$. The angle sum is $180^{\circ}$ if and only if $a, b$ and $c$ are on one great-circle and in a hemisphere. The angle sum is $540^{\circ}$ if and only if $a, b$ and $c$ are on one great-circle and not in a hemisphere.

- The perpendicular bisector of a closed arc $[x \frown y]$ is the great circle that passes through the midpoint of $[x \frown y]$ and is perpendicular to $[x \frown y]$.

- A set $C \subseteq A$ is convex if it contains all the arcs $[x \frown y]$ joining any two points $x, y \in C$. The convex hull of a set $X \subseteq A$ is the set $C o(X)=\cap\{C: X \subseteq C, C$ convex $\}$.

\section{Results}

In this chapter we show for a Pareto optimal rule $\varphi$ which is strict strategy-proof or coalitional strategy-proof, that the set of decisive coalitions is an ultrafilter (see Definition 2.6). By Lemma 2.1, if the set of decisive coalitions for a rule $\varphi$ is an ultrafilter, then $\varphi$ is dictatorial.

Definition 2.6. A collection $\mathscr{D}$ of coalitions is an ultrafilter if it satisfies the following

1. $\emptyset \notin \mathscr{D}$.

2. $S \in \mathscr{D}$ or $N \backslash S \in \mathscr{D}$, for all $S \subseteq N$.

\footnotetext{
${ }^{1}$ This is a familiar approach, [see Kirkman and Sondermann, 1972] and [see Hansson, 1976].
} 
3. If $S, T \in \mathscr{D}$ then $S \cap T \in \mathscr{D}$, for all $S, T \subseteq N$.

Lemma 2.1. Let $\mathscr{D}$ be an ultrafilter on $N$. Then there exists a unique $d \in N$ such that $\{d\} \in \mathscr{D}$.

Proof. Contrary to what we wish to prove let us assume that, there is no such $d$. This implies, $N \backslash\{i\} \in \mathscr{D}$ for all $i \in N$. Then by property (iii) of an ultrafilter, $\bigcap_{i \in N} N \backslash\{i\} \in \mathscr{D}$, but this violates property (i) since $\bigcap_{i \in N} N \backslash\{i\}=\emptyset$. Hence, there must be at least one such $d$.

By property (iii) and (i) there can be only one $d$.

Next, we state the following preliminary result.

Lemma 2.2. Let $\varphi$ be a Pareto optimal rule that is strict strategy-proof or coalitional strategy-proof. Let $\mathscr{D}$ be the set of decisive coalitions for $\varphi$. Then (i) $N \in \mathscr{D}$ and (ii) for each $S \subseteq N$ either $S \in \mathscr{D}$ or $N \backslash S \in \mathscr{D}$.

Proof. (i) We need to show that, if every agent in $N$ has the same peak $a$ (say), then $\varphi\left(a^{N}\right)=a$. It simply follows from Pareto optimality of $\varphi$.

For (ii), note that $\varphi$ is strategy-proof. We present the proof in two parts, in the following Lemmas 2.3 and 2.4.

Lemma 2.3. Let $a$ and $\hat{a}$ be antipodal and $S \subseteq N$. Then $\varphi\left(a^{S}, \hat{a}^{N \backslash S}\right) \in\{a, \hat{a}\}$.

\footnotetext{
${ }^{2}$ Then (ii) follows by similar arguments as the corresponding Lemma 2.5 in ''Ozt'urk, 2013.'Ozt'urk, 2013.'Ozt'urk, 2013. But to show how it is extended in 3-dimension we present the proof.
} 

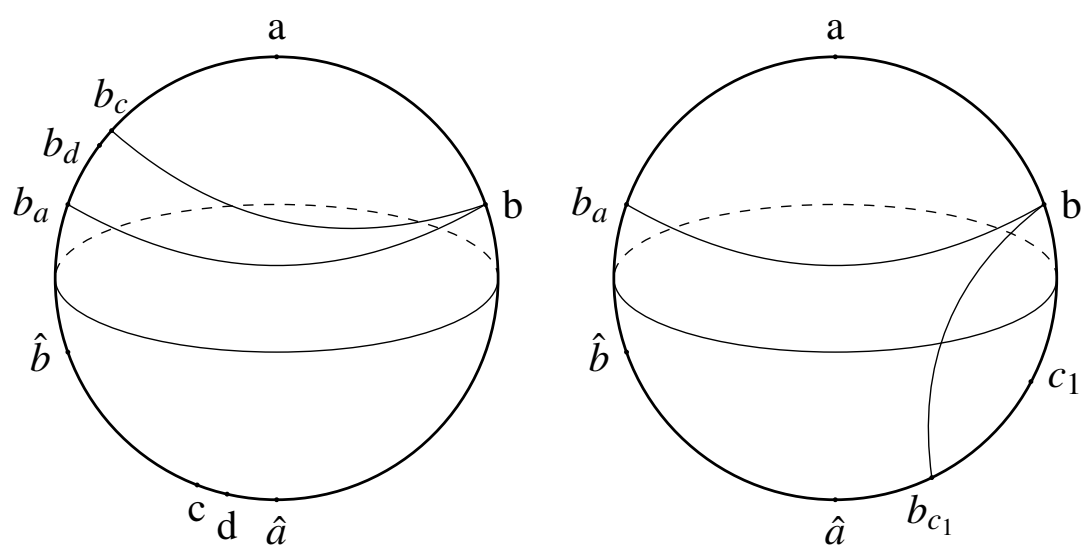

Figure 2.1: Lemma 2.3

Proof. To the contrary of what we wish to prove, assume that $\varphi\left(a^{S}, \hat{a}^{N \backslash S}\right)=b$, where $b \notin$ $\{a, \hat{a}\}$. Without loss of generality we can assume that $|a-b| \leq|\hat{a}-b|$ (on the great circle $\left.G_{a b}\right)$. For $x, y \in A$ let $y_{x}$ be the point on $G_{x y}$ such that $\left\{y, y_{x}\right\}=\left\{z \in G_{x y}:|x-z|=|x-y|\right\}$.

We know, $\varphi\left(a^{S}, \hat{a}^{N \backslash S}\right)=b$. Strategy-proofness implies $\varphi\left(a^{S}, b^{N \backslash S}\right)=b$ and $\varphi\left(b^{S}, \hat{a}^{N \backslash S}\right)=$ $b$. For any $c \in\left[\hat{a} \frown \hat{b}\left[\right.\right.$ we have $\varphi\left(a^{S}, c^{N \backslash S}\right) \in\left[b_{a} \frown b_{c}\right]$ (for $c$ closer to $\hat{a}$ ) or $\varphi\left(a^{S}, c^{N \backslash S}\right) \in$ $\left[b_{a} \frown a\right]$ (for $c$ closer to $\hat{b}$ ) (See Figure 2.Il). Now suppose, $\varphi\left(a^{S}, c^{N \backslash S}\right) \neq b_{a}$ and $\varphi\left(a^{S}, c^{N \backslash S}\right)=d$, where $\left.\left.d \in\right] b_{a} \frown a\right]$. Then $N \backslash S$ can move $c$ towards $\hat{a}$ in such a way that $\varphi\left(a^{S}, c^{N \backslash S}\right) \in\left[b_{a} \frown d\left[\right.\right.$. But this violates strategy-proofness. So, $\varphi\left(a^{S}, \hat{b}^{N \backslash S}\right)=b_{a}$. Since, choice of $c$ was arbitrary $\varphi\left(a^{S}, x^{N \backslash S}\right)=b_{a}$ where $x \in[\hat{a} \frown \hat{b}$. Strategy-proofness now implies $\varphi\left(a^{S}, \hat{b}^{N \backslash S}\right)=b_{a}$.

Now let us consider the case when $S$ moves its peak towards $b$. Take a point $c \in[a \frown b[$. So, Pareto optimality and strategy-proofness imply $\varphi\left(c^{S}, \hat{b}^{N \backslash S}\right)=b_{a}$. Now, if $\varphi\left(b^{S}, \hat{b}^{N \backslash S}\right) \neq b_{a}$, then it violates strategy-proofness. So, $\varphi\left(b^{S}, \hat{b}^{N \backslash S}\right)=b_{a}$.

Next we start with moving the peak of $S$ from $a$ to $b$, we have $\varphi\left(b^{S}, \hat{a}^{N \backslash S}\right)=b$ (See Figure 2.1). Further let us move the peak of $N \backslash S$ from $\hat{a}$ to $\hat{b}$. Take a point $c_{1} \in[\hat{a} \frown \hat{b}[$. Then, $\varphi\left(b^{S}, c_{1}^{N \backslash S}\right)=b$. Now, if $\varphi\left(b^{S}, \hat{b}^{N \backslash S}\right) \neq b$, then $N \backslash S$ with true peak at $c_{1}$ can move to $\hat{b}$ and get better off. This violates strategy-proofness. So, $\varphi\left(b^{S}, \hat{b}^{N \backslash S}\right)=b$.

But $b \neq b_{a}$. This contradiction completes the proof.

The next lemma shows that at two-peak profiles as in Lemma 2.3 , always the same coali- 
tion gets its best point as the outcome.

Lemma 2.4. Let $S \subseteq N$. Then either $\varphi\left(x^{S}, \hat{x}^{N \backslash S}\right)=\hat{x}$ for all $x \in A$ or $\varphi\left(x^{S}, \hat{x}^{N \backslash S}\right)=x$ for all $x \in A$.

Proof. Let $a, b \in A$. In view of Lemma 2.3, we can assume without loss of generality that $\varphi\left(a^{S}, \hat{a}^{N \backslash S}\right)=\hat{a}$. It is sufficient to prove that $\varphi\left(b^{S}, \hat{b}^{N \backslash S}\right)=\hat{b}$. We distinguish two cases.

Case 2.1. $\{a, \hat{a}\} \cap\{b, \hat{b}\}=\emptyset$

Contrary to what we wish to prove let us assume that $\varphi\left(b^{S}, \hat{b}^{N \backslash S}\right) \neq \hat{b}$. Then Lemma 2.3 yields $\varphi\left(b^{S}, \hat{b}^{N \backslash S}\right)=b$. Strategy-proofness and $\varphi\left(a^{S}, \hat{a}^{N \backslash S}\right)=\hat{a}$ imply $\varphi\left(b^{S}, \hat{a}^{N \backslash S}\right)=\hat{a}$. But strategy-proofness and $\varphi\left(b^{S}, \hat{b}^{N \backslash S}\right)=b$ imply $\varphi\left(b^{S}, \hat{a}^{N \backslash S}\right)=b$. This yields the contradiction $b=\hat{a}$.

Case 2.2. $b=\hat{a}$

So, $\{a, \hat{a}\}=\{b, \hat{b}\}$. Take $c \in A \backslash\{a, \hat{a}, b, \hat{b}\}$. Applying the previous case yields first $\varphi\left(c^{S}, \hat{c}^{N \backslash S}\right)=\hat{c}$ and by this result that $\varphi\left(b^{S}, \hat{b}^{N \backslash S}\right)=\hat{b}$.

Hence this completes the proof.

Lemma 2.5. Let $S \subset N$. Then either $S$ is decisive or $N \backslash S$ is decisive.

Proof. In view of Lemma 2.4, we can assume without loss of generality that $\varphi\left(a^{S}, \hat{a}^{N \backslash S}\right)=$ $a \forall a \in A$. We prove that $S$ is decisive. Take $b \in A$ and $p \in A^{N}$ such that $p(i)=b \forall i \in S$. It is sufficient to prove that $\varphi(p)$ is at minimal distance from $b$.

By assumption $\varphi\left(b^{S}, \hat{b}^{N \backslash S}\right)=b$. Now strategy-proofness implies $\varphi(p)=b$.

The remainder of the chapter will concentrate on proving property (iii) of an ultrafilter for $\varphi$, i.e. if $S, T \in \mathscr{D}$ then $S \cap T \in \mathscr{D}$, for all $S, T \subseteq N$. But before delving into that, let us see the implication of Pareto optimality on $\varphi$.

Lemma 2.6. Let $G$ be a great circle and let $H$ be one of the hemispheres induced by $G$. Let $p \in A^{N}$ be such that $p(i) \in G \cup H$ for all $i \in N$, and $p(i) \in H$ for some $i \in N$. Then $t \in A$ is Pareto optimal for $p$ if and only if $t \in \operatorname{Co}(\{p(1), p(2), \ldots, p(n)\})$.

Proof. Only if part: 
Let us assume that $t$ is Pareto optimal. We need to show that $t \in$ $\operatorname{Co}(\{p(1), p(2), \ldots, p(n)\})$. To the contrary suppose that does not happen, i.e. $t \notin C o(\{p(1), p(2), \ldots, p(n)\})$. Then we can separate $t$ and $C o(\{p(1), p(2), \ldots, p(n)\})$ by a great circle. Suppose $p(i)$ is closest peak from $t$ and let $L$ be the perpendicular bisector of $[p(i) \frown t]$. All the peaks are in the hemisphere $(L, p(i))$. So, $p(i)$ is closer to all the peaks than $t$. This contradicts our initial assumption that $t$ is Pareto optimal.

If part:

Let us assume that, $t \in C o(\{p(1), p(2), \ldots, p(n)\})$. We need to show that $t$ is Pareto optimal. Take any other point $y$ and let $L_{1}$ be the perpendicular bisector of $[t \frown y]$. Since, $t \in \operatorname{Co}(\{p(1), p(2), \ldots, p(n)\})$ we must have $p(j) \in\left(L_{1}, t\right)$ for some $j \in N$. Hence, moving the output from $t$ to $y$ is worse for agent $j$. Since, the choice of $y$ was arbitrary we conclude that $t$ is Pareto optimal.

\section{Strict Strategy-proofness and Coalitional Strategy-proofness}

In this section we show that for a Pareto optimal rule the set of decisive coalitions is closed under intersection if the rule is strict strategy-proof or coalitional strategy-proof. We start with considering strict strategy-proofness. In the proof of the following proposition, we use the easily established facts that strict strategy-proofness implies intermediate strategyproofness and intermediate strict strategy-proofness.

Proposition 2.1. Let $\varphi$ be Pareto optimal and strict strategy-proof, and let $S$ and $T$ be decisive coalitions. Then $S \cap T$ is decisive. Hence, under the stated conditions, $\mathscr{D}$ is an ultrafilter.

Proof. Contrary to what we wish to prove, suppose that $S \cap T$ is not decisive. Let $X=S \backslash T$, $Y=S \cap T$, and $Z=N \backslash S$. Hence $X \cup Y=S$ and $Y \cup Z=N \backslash(S \backslash T) \supseteq T$ are decisive since $S$ and $T$ are decisive, and $X \cup Z=N \backslash(S \cap T)$ is decisive by Lemma 2.5.

Let $G$ be a great circle with equidistant points $a, b$, and $c$, and let $p \in A^{N}$ with $p(i)=a$ for all $i \in X, p(i)=b$ for all $i \in Y$, and $p(i)=c$ for all $i \in Z$. We denote $p=\left(a^{X}, b^{Y}, c^{Z}\right)$. We first show that $\varphi(p) \notin G$.

Since $X \cup Y$ is decisive, hence $\varphi\left(b^{X \cup Y}, c^{Z}\right)=b$ and $\varphi\left(a^{X \cup Y}, c^{Z}\right)=a$, it follows by strict 
strategy-proofness that

$$
\begin{aligned}
& |a-\varphi(p)|<|a-b| \text { or } \varphi(p)=b \\
& \text { and }|b-\varphi(p)|<|b-a| \text { or } \varphi(p)=a
\end{aligned}
$$

Similarly, since $X \cup Z$ and $Y \cup Z$ are decisive, we derive

$$
\begin{array}{r}
|c-\varphi(p)|<|c-a| \text { or } \varphi(p)=a \\
\text { and }|a-\varphi(p)|<|a-c| \text { or } \varphi(p)=c
\end{array}
$$

and

$$
\begin{aligned}
& |c-\varphi(p)|<|c-b| \text { or } \varphi(p)=b \\
& \text { and }|b-\varphi(p)|<|b-c| \text { or } \varphi(p)=c \text {. }
\end{aligned}
$$

If $\varphi(p)=a$, then (2.3) is violated, hence $\varphi(p) \neq a$, and similarly $\varphi(p) \neq b$ and $\varphi(p) \neq c$. If $\varphi(p) \in(a \frown b)$, then (2.2) is violated, hence $\varphi(p) \notin(a \frown b)$. Similarly, $\varphi(p) \notin(a \frown c)$ and $\varphi(p) \notin(b \frown c)$. Therefore, $\varphi(p) \notin G$.

Let $H_{1}$ and $H_{2}$ be the two hemispheres separated by $G$. Assume without loss of generality that $\varphi(p) \in H_{1}$. For any $x \in A$, we denote the profile $\left(x^{X}, b^{Y}, c^{Z}\right)$ by $p^{x}$, so $p=p^{a}$. Consider a sequence of points $\left(d_{k}\right)_{k \in \mathbb{N}} \in H_{2}$ converging to $a$. By Lemma $2.6, f^{k}:=\varphi\left(p^{d_{k}}\right) \in H_{2} \cup G$ for every $k \in \mathbb{N}$. Since $H_{2} \cup G$ is compact we may assume that $\left(f^{k}\right)_{k \in \mathbb{N}}$ converges to some point $f^{*} \in H_{2} \cup G$. Now $f^{*} \neq a$, otherwise $\left|f^{k}-a\right|<|\varphi(p)-a|$ for large $k$, so $X$ could manipulate via $p^{d_{k}}$ at $p$, contradicting intermediate strategy-proofness.

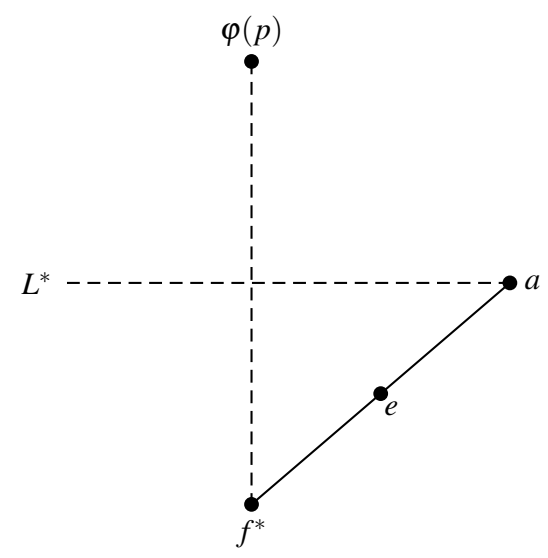

Figure 2.2: Second part of the proof of Proposition 2.1 
Let the great circle $L^{*}$ be the perpendicular bisector of $\left[\varphi(p) \frown f^{*}\right]$. Let $\left(L^{*}, f^{*}\right)$ and $\left(L^{*}, \varphi(p)\right)$ denote the two hemispheres induced by $L^{*}$ and containing the points $f^{*}$ and $\varphi(p)$, respectively. If $a \in\left(L^{*}, f^{*}\right)$, then for large $k$ we have $|a-\varphi(p)|>\left|a-f^{k}\right|$, so that as before $X$ can manipulate at $p$ via $p^{d_{k}}$. If $a \in\left(L^{*}, \varphi(p)\right)$, hence $|a-\varphi(p)|<\left|a-f^{*}\right|$, then for large $k$ we have $\left|d_{k}-\varphi(p)\right|<\left|d_{k}-f^{k}\right|$ : then $X$ can manipulate at $p^{d_{k}}$ via $p$, contradicting again intermediate strategy-proofness. Hence $a \in L^{*}$.

Let $e \in\left(f^{*} \frown a\right)$ (See Figure 2.2). Since $|a-\varphi(p)|=\left|a-f^{*}\right|$ we have $\varphi\left(p^{e}\right) \neq f^{*}$, otherwise intermediate strict strategy-proofness would be violated at $p$ via $p^{e}$.

Let $e_{k} \in\left(f^{k} \frown d_{k}\right), k \in \mathbb{N}$, such that $\left(e_{k}\right)_{k \in \mathbb{N}}$ converges to $e$. For every $k \in \mathbb{N}$, by intermediate strategy-proofness, $\left|\varphi\left(p^{e_{k}}\right)-e^{k}\right| \leq\left|\varphi\left(p^{d_{k}}\right)-e^{k}\right|$, otherwise $X$ could manipulate at $p^{e_{k}}$ via $p^{d_{k}}$; and $\left|\varphi\left(p^{d_{k}}\right)-d_{k}\right| \leq\left|\varphi\left(p^{e_{k}}\right)-d_{k}\right|$, otherwise $X$ could manipulate at $p^{d_{k}}$ via $p^{e_{k}}$. Since $e_{k} \in\left(f^{k} \frown d_{k}\right)$, we obtain $\varphi\left(p^{e_{k}}\right)=\varphi\left(p^{d_{k}}\right)=f^{k}$ for every $k \in \mathbb{N}$. By similar arguments as before for the point $a$, it follows that $e$ is on the perpendicular bisector $L^{* *}$ of $\left[f^{*} \frown \varphi\left(p^{e}\right)\right]$. Hence, $\left|e-\varphi\left(p^{e}\right)\right|=\left|e-f^{*}\right|$. But then

$$
\begin{aligned}
|a-\varphi(p)| & =\left|a-f^{*}\right|\left(\text { since } a \in L^{*}\right) \\
& =\left|f^{*}-e\right|+|a-e|\left(\text { since } e \in\left(f^{*} \frown a\right)\right) \\
& \left.=\left|\varphi\left(p^{e}\right)-e\right|+|a-e| \text { (since } e \in L^{* *}\right) \\
& \geq\left|\varphi\left(p^{e}\right)-a\right| \text { (by triangular inequality). }
\end{aligned}
$$

Hence, $|a-\varphi(p)| \geq\left|\varphi\left(p^{e}\right)-a\right|$, which by intermediate strict strategy-proofness implies $\varphi\left(p^{e}\right)=\varphi(p)$, and thus $e \in L^{*}$, contradicting $e \in\left(f^{*} \frown a\right)$.

We can now state our first main result.

Corollary 2.1. Let $\varphi$ be Pareto optimal and strict strategy-proof. Then $\varphi$ is dictatorial.

Proof. By Lemma 2.2 and Proposition 2.1, the set of decisive coalitions for $\varphi$ is an ultrafilter. Hence $\varphi$ is dictatorial.

We proceed by considering coalitional strategy-proofness. 
Proposition 2.2. Let $\varphi$ be Pareto optimal and coalitional strategy-proof, and let $S$ and $T$ be decisive coalitions. Then $S \cap T$ is decisive. Hence, under the stated conditions, $\mathscr{D}$ is an ultrafilter.

Proof. Contrary to what we wish to prove, suppose that $S \cap T$ is not decisive. Let $X$, $Y$, and $Z$ be as in the proof of Proposition 2.1: as there, $X \cup Y, Y \cup Z$, and $X \cup Z$ are decisive. Consider a profile $p=\left(a^{X}, b^{Y}, c^{Z}\right)$ where $a, b, c \in A$ are distinct points in the same hemisphere and such that the angles in the triangle with edges $[a \frown b],[b \frown c]$, and $[a \frown c]$ are less than $90^{\circ}$. By Remark 2.6, $\varphi(p) \in C o(\{a, b, c\})$.

Suppose $\varphi(p) \notin[a \frown b]$. Let $m$ be the point of $[a \frown b]$ closest to $\varphi(p)$. Then $|a-m|<$ $|a-\varphi(p)|$ and $|b-m|<|b-\varphi(p)|$, so that $X \cup Y$ can manipulate at $p$ via $\left(m^{X \cup Y}, c^{Z}\right)$. This is a violation of coalitional strategy-proofness. Thus, $\varphi(p) \in[a \frown b]$. Similarly one shows that $\varphi(p) \in[c \frown a]$ and $\varphi(p) \in[b \frown c]$, but this is not possible. We conclude that $S \cap T$ is decisive.

By this Proposition 2.2 and Lemma 2.2 we obtain our second main result.

Corollary 2.2. Let $\varphi$ be Pareto optimal and coalitional strategy-proof. Then $\varphi$ is dictatorial.

It is an open problem whether Pareto optimality and strategy-proofness imply dictatorship. If not, coordinate median rules would be the typical candidate rules. The following example, however, suggests that such rules will be manipulable.

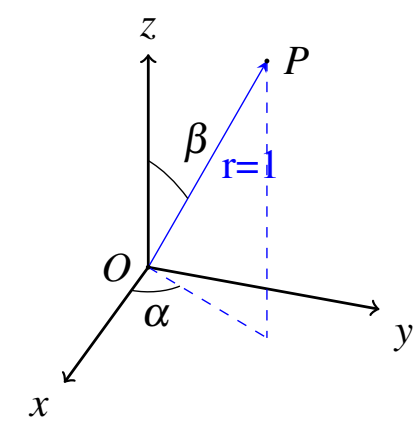

Figure 2.3: Spherical Coordinate System

Before defining a coordinatewise rule we need to define a spherical coordinate system. For that we have to choose two orthogonal axes, the polar and the azimuth reference, and an 
origin point in space. These choices determine a reference plane that contains the origin and is perpendicular to the polar direction. The spherical coordinates $(\alpha, \beta)$ of a point $P$ (see Figure 2.3) are then defined as follows:

- $\alpha$ : The azimuthal angle is the signed angle measured from the azimuth reference direction to the orthogonal projection of the line segment $O P$ on the reference plane.

- $\beta$ : The polar angle is the angle between the $Z$-axis and the line segment $O P$.

Simple conversion gives the Euclidean coordinate of point $P$ as $(\sin \beta \cos \alpha, \sin \beta \sin \alpha, \cos \beta)$. A coordinatewise median rule $F_{\text {coord }}$ takes coordinates of all the reported peaks $\left(\alpha_{1}, \beta_{1}\right),\left(\alpha_{2}, \beta_{2}\right), \ldots,\left(\alpha_{n}, \beta_{n}\right)$ and then computes medians of both the polar angles median $\left(\beta_{1}, \beta_{2}, \ldots, \beta_{n}\right)=\beta_{\text {med }}$ and the azimuth angles $\operatorname{median}\left(\alpha_{1}, \alpha_{2}, \ldots, \alpha_{n}\right)=\alpha_{m e d}$. So,

$$
F_{\text {coord }}(p(1), p(2), \ldots, p(n))=F_{\text {coord }}\left(\left(\alpha_{1}, \beta_{1}\right),\left(\alpha_{2}, \beta_{2}\right), \ldots,\left(\alpha_{n}, \beta_{n}\right)\right)=\left(\alpha_{\text {med }}, \beta_{\text {med }}\right) .
$$

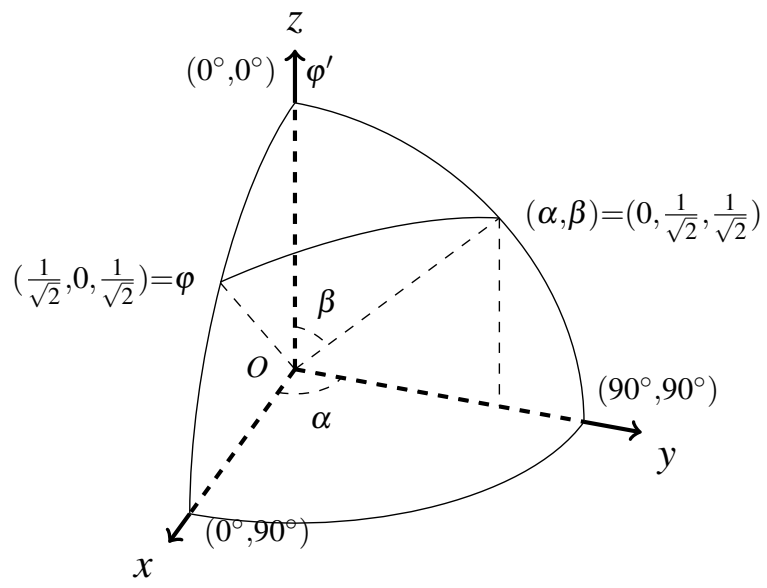

Figure 2.4: Example

Example 2.1. In order to stay on the sphere while taking coordinatewise medians, we express the points of $A$ by two coordinates, namely the angle with the vector $(1,0,0)$ and the angle with the vector $(0,0,1)$, both in degrees. Now suppose there are three agents with peaks $p(1)=\left(0^{\circ}, 0^{\circ}\right), p(2)=\left(0^{\circ}, 90^{\circ}\right)$, and $p(3)=\left(90^{\circ}, 45^{\circ}\right)$. In Euclidian coordinates these are the points $(0,0,1),(1,0,0)$, and $\left(0, \frac{1}{2} \sqrt{2}, \frac{1}{2} \sqrt{2}\right)$, respectively (see Figure 
2.4). The coordinatewise median rule assigns the point $\left(0^{\circ}, 45^{\circ}\right)$, hence $\left(\frac{1}{2} \sqrt{2}, 0, \frac{1}{2} \sqrt{2}\right)$ in Euclidian coordinates. If agent 3 reports $\tilde{p}(3)=\left(90^{\circ}, 0^{\circ}\right)$ instead, then the coordinatewise median rule would assign the point $\left(0^{\circ}, 0^{\circ}\right)$ or $(0,0,1)$, and it is easy to check that this is closer to agent 3 's true peak $\left(0, \frac{1}{2} \sqrt{2}, \frac{1}{2} \sqrt{2}\right)$ than the sincere outcome $\left(\frac{1}{2} \sqrt{2}, 0, \frac{1}{2} \sqrt{2}\right)$ is. Thus, agent 3 manipulates successfully. 



\section{CHAPTER 3}

\section{Locating Two Public Goods in $\mathbb{R}^{2}$}

\section{Introduction}

The central problem of this chapter is to locate two public goods in a two dimensional region. These two goods are identical, for instance, a pair of hospitals or a pair of libraries, but not a hospital and a library. Researchers have already studied the problem of locating two public goods on a street represented by a straight line (see [Miyagawa, 2001], [Ehlers, 2001], [Ehlers, 2002], [Heo, 2013], [Gordon, 2007]). Since the object is a public good it is assumed that people (agents) have single peaked preferences for them, i.e., for an agent there exists a point which is her most preferred point (peak) to locate one good and her preference decreases with the increase of the distance of the chosen location and her peak. Here we need to locate two goods, therefore we combine the (marginal) preferences for the location of one good to find a joint preference over a pair of locations. Researchers dealing with this problem on a straight line have used several types of joint preferences, Maxextension and Lexmax-extension are two popular ones. For Max extension it is assumed that an agent cares only about the location of the good which is closest to her peak. So, her preference for a pair of location remains the same as long as the nearest public good to her does not move. In case of Lexmax extension an agent cares about both the locations 
but she is most concerned about the closest one. Therefore, unlike Max extension here an agent can prefer a pair of locations over another pair even if the closest ones are at the same distance from her peak and the farther ones are at different distances. In this paper we restrict our attention to Lexmax preferences. The social planner has to device a rule which takes agents' preferences as inputs and chooses a pair of locations. We impose a strategyproofness condition on the rule to ensure that they are not manipulable by any single agent. Moreover we do not want the rule to be sensitive of extreme preferences and that is ensured by a uncompromisingness condition. We impose peaks-onlyness of the social choice function implying that it depends only on agents' peaks(top-ranked alternative) ignoring the whole preference. We also impose continuity of the social choice function with respect to the peaks. We impose a condition called box-property which means that both the outcomes are chosen from the smallest axis-aligned rectangle containing all the peaks. In section $[3.3$ we derive the uncompromisingness condition from other conditions. In section [3.5 we characterise a class of rules called double generalised median rules. In section 3.6 we discuss interdependence of the conditions used in the characterisation result. It is still an open problem to check whether all conditions are independent or not.

In the following section 3.2 we introduce the formal model.

\section{Model}

We have a finite set of agents denoted by $N=\{1, \ldots, n\}$. Agents in $N$ are interested in the two dimensional Euclidean space denoted by $\mathbf{R}^{2}$. We assume that there is a given coordinate system, i.e., an $X$-axis and a $Y$-axis. For every point $t \in \mathbf{R}^{2}$ we denote its $X$ coordinate by $t_{1}$ and its $Y$ coordinate by $t_{2}$. Throughout the chapter we use superscripts for agents and subscripts for coordinates.

For any point $t \in \mathbf{R}^{2}$, four open quadrants are created by considering $t$ as the origin and having an axis parallel to the original $X$-axis and another axis parallel to the original $Y$-axis. We denote these quadrants by $Q_{++}(t), Q_{-+}(t), Q_{--}(t), Q_{+-}(t)$, where the first subscript under $Q$ is for $X$-axis and the second subscript is for $Y$-axis. A + symbol in the first subscript means the region under consideration contains points having $X$-coordinate larger than $t_{1}$. Straight lines separating the quadrants are denoted by $Q_{0+}(t), Q_{0-}(t), Q_{+0}(t), Q_{-0}(t)$, where a 0 in the first subscript indicates a vertical straight line and a 0 in the second subscript indicates a horizontal straight line. 


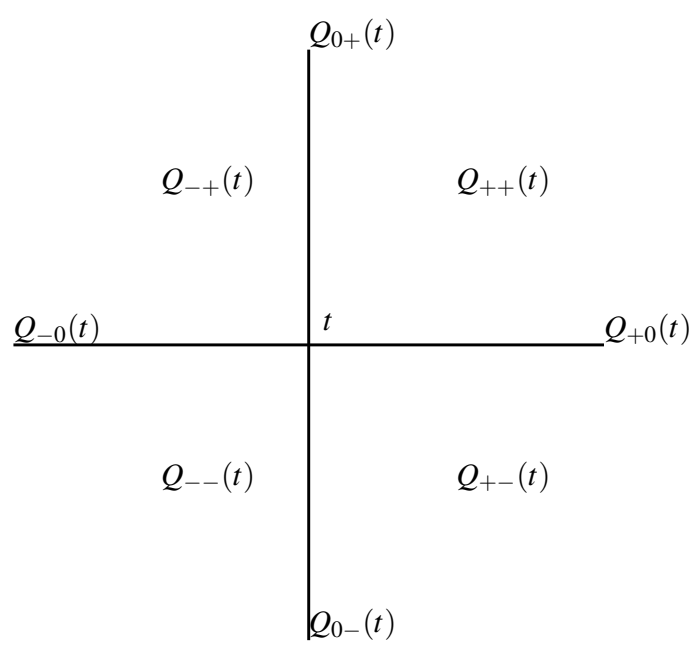

Figure 3.1: Quadrants with center at $t \in \mathbf{R}^{2}$

We denote the corresponding closed regions by $\bar{Q}_{++}(t), \bar{Q}_{+-}(t), \bar{Q}_{-+}(t), \bar{Q}_{--}(t)$, defined as $\bar{Q}_{++}(t)=Q_{++}(t) \cup Q_{+0}(t) \cup Q_{0+}(t) \cup\{t\}$ and so on. Also, $\bar{Q}_{0+}=Q_{0+} \cup\{t\}$ and so on.

Since, the problem is about a pair of locations, the set of alternatives is $\mathbf{R}^{\mathbf{2}} \times \mathbf{R}^{\mathbf{2}}$, which we denote by A. Agents' preferences for a pair of locations are based on their preferences for a single location. To distinguish between these two preferences, we call the former one the joint preference and the latter one the marginal preference. Marginal preference relations are complete and transitive. Marginal preferences are single peaked with quadratically separable utilities ${ }^{\boxplus}$ for every agent $i \in N$. Thus, for an agent $i \in N$, there is a peak $p^{i} \in \mathbf{R}^{\mathbf{2}}$ and a pair of non-negative weights $\alpha_{1}^{i}, \alpha_{2}^{i}$, which define his utility function:

$$
u^{i}\left(x ; p^{i}, \alpha^{i}\right)=-\sqrt{\alpha_{1}^{i}\left(x_{1}-p_{1}^{i}\right)^{2}+\alpha_{2}^{i}\left(x_{2}-p_{2}^{i}\right)^{2}}
$$

So, a tuple $\left(p^{i} ; \alpha_{1}^{i}, \alpha_{2}^{i}\right)$ fully characterises the marginal preference of agent $i$. For a fixed marginal preference we simply use $u^{i}(x)$ to denote utility of agent $i$ from a location at $x$. Agent $i$ weakly prefers $x$ to $y$ if $u^{i}(x) \geqslant u^{i}(y)$, and the preference is strict in case of strict inequality. $U\left(t \mid p^{i}, \alpha^{i}\right)=\left\{x \in \mathbf{R}^{\mathbf{2}}: u^{i}\left(x ; p^{i}, \alpha^{i}\right) \geqslant u^{i}\left(t ; p^{i}, \alpha^{i}\right)\right\}$ denotes the set of points having utility at least as much as $t$ with respect to $\left(p^{i}, \alpha^{i}\right)$.

\footnotetext{
${ }^{1}$ [Border and Jordan, 1983] uses star-shaped quadratically separable preferences on $\mathbf{R}^{\mathbf{m}}$.
} 
Joint preference relation of agent $i$ is denoted by $R^{i}$. For any point $x \in \mathbf{R}^{2}$ we define $\bar{x}$ as follows.

$$
\bar{x}=\left\{\begin{array}{l}
\left(x_{1}, x_{2}\right) \text { if } x_{1} \geqslant x_{2} \\
\left(x_{2}, x_{1}\right) \text { otherwise }
\end{array}\right.
$$

For any two $x, y \in \mathbf{R}^{2}, x$ is lexicographically (weakly) better than $y$ if $\bar{x}_{1}>\bar{y}_{1}$ or $\bar{x}_{1}=\bar{y}_{1}$ and $\bar{x}_{2}>\bar{y}_{2}$. Joint lexmax preference relation $R^{i}$ is defined as follows. Consider two pairs of locations $(x, y) \in \mathbf{A}$ and $(v, w) \in \mathbf{A}$. Then, $(x, y) R^{i}(v, w)$ if $\left(u^{i}(x), u^{i}(y)\right)$ is lexicographically better than $\left(u^{i}(v), u^{i}(w)\right) . P$ denotes the strict part of $R$.

A profile of preferences is denoted by $R$, where $R=\left(R^{1}, \ldots, R^{n}\right)$, with $R^{i} \in \mathscr{R}$ for all $i \in N$. A profile is often written as $R=\left(R^{i}, R^{-i}\right)$, where $R^{-i}$ is preference profile of all agents' except agent $i$. A rule is a function that takes preference profiles as inputs and its output is a pair of locations in A. Hence, a rule denoted by $F$ is a function $F: \mathscr{R}^{n} \rightarrow \mathbf{A}$.

$\mathscr{R}$ denotes the set of all possible preferences. A profile of peaks is denoted by $p$, where $p=\left(p^{1}, \ldots, p^{n}\right)$, with $p^{i} \in \mathbf{R}^{2}$ being the peak of corresponding to agent $i^{\prime} s$ preference $R^{i}$. The smallest axis-aligned rectangle containing all the reported peaks is called the box of the peaks profile $p$. It is defined as

$$
\operatorname{box}(p)=\left\{x \in \mathbf{R}^{\mathbf{2}} \mid \min _{i} p_{1}^{i} \leqslant x_{1} \leqslant \max _{i} p_{1}^{i}, \min _{i} p_{2}^{i} \leqslant x_{2} \leqslant \max _{i} p_{2}^{i}\right\}
$$

For a preference profile $R, F(R)$ is a pair of locations denoted by $F^{A}(R)$ and $F^{B}(R)$ or simply by $F^{A}$ and $F^{B}$ when there is no confusion. Let $\underline{a}=\inf _{R \in \mathscr{R}^{n}} F_{1}^{A}(R)$ if this infimum exists and $\underline{a}=-\infty$ otherwise. Similarly, let $\bar{a}=\sup _{R \in \mathscr{R}^{n}} F_{1}^{A}(R)$ if this supremum exists and $\bar{a}=\infty$ otherwise. Similarly, $\underline{b}=\inf _{R \in \mathscr{R}^{n}} F_{1}^{B}(R)$ if this infimum exists and $\underline{b}=-\infty$ otherwise. And $\bar{b}=\sup _{R \in \mathscr{R}^{n}} F_{1}^{B}(R)$ if this supremum exists and $\bar{b}=\infty$ otherwise.

Based on $F^{A}$ and $F^{B}$ we partition $\mathbf{R}^{2}$ in at most 9 open sets or regions. These 9 open sets are denoted by $I_{1}, \ldots, I_{9}$. Suppose that $F^{A}(p) \in Q_{--}\left(F^{B}(p)\right)$. Then these 9 sets are defined as follows, $I_{1}=Q_{-+}\left(F^{A}\right) \cap Q_{-+}\left(F^{B}\right), I_{2}=Q_{++}\left(F^{A}\right) \cap Q_{-+}\left(F^{B}\right), I_{3}=Q_{++}\left(F^{A}\right) \cap$ $Q_{++}\left(F^{B}\right), I_{4}=Q_{-+}\left(F^{A}\right) \cap Q_{--}\left(F^{B}\right), I_{5}=Q_{++}\left(F^{A}\right) \cap Q_{--}\left(F^{B}\right), I_{6}=Q_{++}\left(F^{A}\right) \cap$ $Q_{+-}\left(F^{B}\right), I_{7}=Q_{--}\left(F^{A}\right) \cap Q_{--}\left(F^{B}\right), I_{8}=Q_{+-}\left(F^{A}\right) \cap Q_{--}\left(F^{B}\right), I_{9}=Q_{+-}\left(F^{A}\right) \cap$ $Q_{+-}\left(F^{B}\right)$. These regions are shown in the following Figure B.2. 


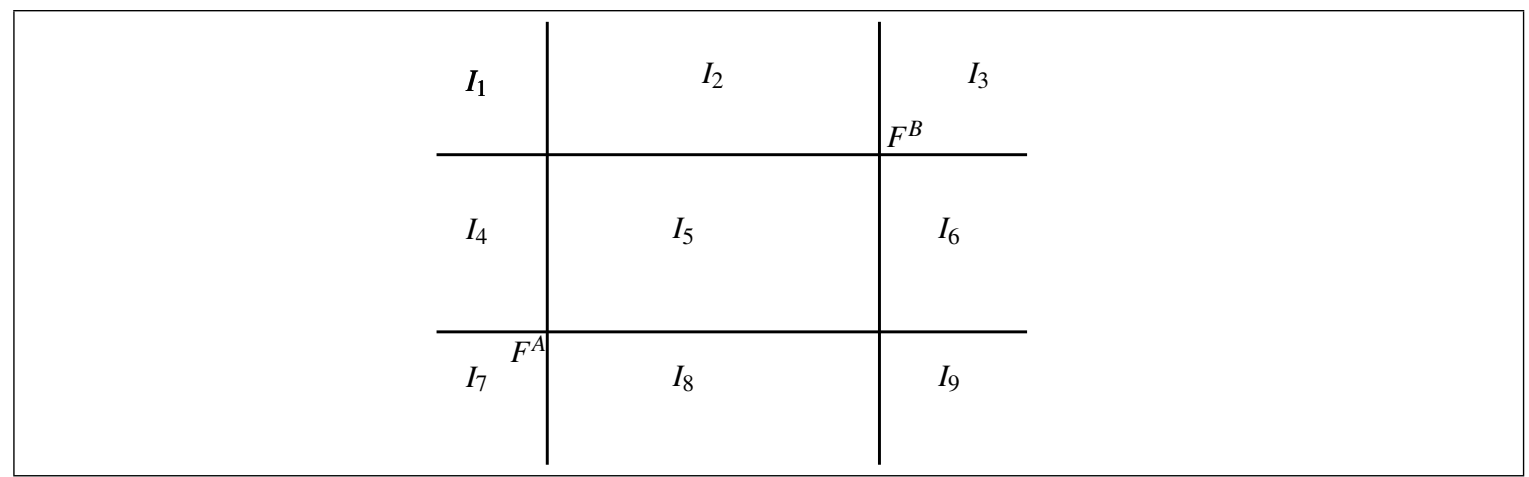

Figure 3.2: Partition of $\mathbf{R}^{2}$ based on $F^{A}$ and $F^{B}$.

Now we define some properties that we impose on the rule.

Definition 3.1. A rule $F$ is said to be peaks only if $F(R)=F\left(R^{\prime}\right)$, whenever $R$ and $R^{\prime}$ have the same profile of peaks, i.e., $p=p^{\prime}$.

As the name suggests output of a peaks-only rule remains unchanged as long as the peaks profile is unchanged. A peaks-only rule can be written as a function of only the peaks, i.e., $F(p)$ instead of $F(R)$.

Definition 3.2. A rule $F$ is said to be continuous with respect to the peaks if both $F^{A}(R)$ and $F^{B}(R)$ are continuous functions of the peaks $p$ with respect to the Euclidean metric in $\mathbf{R}^{2}$.

Due to continuity, outcomes of the rule do not jump when the profile of peaks changes slightly.

Definition 3.3. A rule $F$ is said to be strategy-proof if $F\left(R^{i}, R^{-i}\right) R^{i} F\left(R^{\prime i}, R^{-i}\right)$ for all $i \in N$ and for all $R^{i}$ and $R^{\prime i}$ in $\mathscr{R}^{n}$.

By strategy-proofness of a rule we ensure that truth telling is the dominant strategy for each agent.

Definition 3.4. Let $R^{i}=\left(p^{i}, \alpha\right)$ and $R^{\prime i}=\left(p^{i}, \alpha^{\prime i}\right)$. A rule $F$ is said to be Maskin monotonic if $U\left(F^{A}(R) \mid p^{\prime i}, \alpha^{\prime i}\right) \subseteq U\left(F^{A}(R) \mid p^{i}, \alpha^{i}\right)$ implies $F^{A}\left(R^{\prime i}, R^{-i}\right)=F^{A}(R)$ and $U\left(F^{B}(R) \mid p^{\prime i}, \alpha^{\prime i}\right) \subseteq U\left(F^{B}(R) \mid p^{i}, \alpha^{i}\right)$ implies $F^{B}\left(R^{\prime i}, R^{-i}\right)=F^{B}(R)$. 
Note, that here the monotonicity condition is applied separately for two locations. Suppose that $(t, u)$ is the pair of locations chosen at preference profile $R$ according to the rule $F$ in question. Then, if in any agents'ranking $t$ doesn't fall relative to any other alternative in going from preference profile $R$ to preference profile $R^{\prime}$, Maskin monotonicity requires that $t$ is also chosen at preference profile $R^{\prime}$ and similarly, if $u$ doesnt fall in anyones ranking relative to any other alternative in going from preference profile $R$ to preference profile $R^{\prime}$, Maskin monotonicity requires that $u$ also be chosen in preference profile $R^{\prime}$. In case of a single location in one dimension strategy-proofness implies Maskin monotonicity.

Definition 3.5. Rule $F$ is called double uncompromising if the following is satisfied for any two preference profiles $R, R^{\prime} \in \mathscr{R}^{n}$ and for any agent $i \in N$.

Suppose, peaks $p^{i}$ and $p^{\prime i}$ corresponding to $R$ and $R^{\prime}$ are such that

1. For $h, v \in\{+,-, 0\}, p^{i} \in Q_{h v}\left(F^{A}(R)\right)$ implies $p^{i} \in \bar{Q}_{h v}\left(F^{A}(R)\right)$, then $F^{A}\left(R^{\prime}\right)=$ $F^{A}(R)$;

2. For $h^{\prime}, v^{\prime} \in\{+,-, 0\}, p^{i} \in Q_{h^{\prime} v^{\prime}}\left(F^{B}(R)\right)$ implies $p^{\prime i} \in \bar{Q}_{h^{\prime} v^{\prime}}\left(F^{B}(R)\right)$, then $F^{B}\left(R^{\prime}\right)=$ $F^{B}(R)$.

Double uncompromisingness means that as long as $p^{i}$ and $p^{i}$ are in the same quadrant with respect to $F^{A}(R)$, the new location $F^{A}\left(R^{\prime i}, R^{-i}\right)$ is the same as $F^{A}(R)$, and the same is true for $F^{B}(p)$. A double uncompromising rule is immune to extreme individual reports.

Definition 3.6. A rule $F$ is said to be unanimous iffor all preference profiles $R \in \mathscr{R}^{n}$ with peaks profile $p,\left|\left\{p^{i} \mid i \in N\right\}\right|=1$ implies that $F^{A}(R)=F^{B}(R)=p^{i}$.

Thus, unanimity means that whenever all the peaks are at one point, that point is chosen for both the outcomes.

Definition 3.7. A rule $F$ is said to satisfy the box property if for all preference profiles $R \in \mathscr{R}^{n}$ with peaks profile $p, F^{A}(R), F^{B}(R) \in \operatorname{box}(p)$.

We show in Lemma 3.4 that box property is implied by unanimity and double uncompromisingness.

Definition 3.8. The Option set for agent $i$ under a rule $F$ is the set of all possible outcomes of $F$ resulting from all possible preferences of $R^{i} \in \mathscr{R}$ agent $i$, given the preferences of all other players $R^{-i}$. It is denoted by $O^{i}\left(F ; R^{-i}\right)$, therefore $O^{i}\left(F ; R^{-i}\right)=\{(x, y) \in \mathbf{A} \mid x=$ $F^{A}\left(R^{i}, R^{-i}\right), y=F^{B}\left(R^{i}, R^{-i}\right)$ for some $\left.R^{i} \in \mathscr{R}\right\}$.

In the next section we show implications of these properties on a rule. 


\section{Implications of the Properties}

Lemma 3.1 shows that due to double uncompromisingness, if an agent moves her peak only in one coordinate then the chosen locations' movement in that coordinate is bounded. Lemma 3.2 shows that if an agent moves her peak only in one coordinate then the closest location to the peak cannot move in the other coordinate.

Lemma 3.1. Suppose $F$ is a double uncompromisingness rule and $R, R^{\prime} \in \mathscr{R}^{n}$ are two profiles with $R^{j}=R^{\prime j}$ for all $j \in N \backslash\{i\}$. Let $p, p^{\prime}$ be the profiles of peaks with respect to $R$ and $R^{\prime}$. Suppose agent $i$ 's peak is such that $p_{1}^{\prime i}>p_{1}^{i}$ and $p_{2}^{i}=p_{2}^{i}$. Then,

1. $\min \left(F_{1}^{A}(R), p_{1}^{i}\right) \leqslant F_{1}^{A}\left(R^{\prime}\right) \leqslant \max \left(F_{1}^{A}(R), p_{1}^{\prime i}\right)$ and

2. $\min \left(F_{1}^{B}(R), p_{1}^{i}\right) \leqslant F_{1}^{B}\left(R^{\prime}\right) \leqslant \max \left(F_{1}^{B}(R), p_{1}^{\prime i}\right)$.

Proof. 1. Suppose, for the sake of contradiction, that $F_{1}^{A}\left(R^{\prime}\right)<\min \left(F_{1}^{A}(R), p_{1}^{i}\right)$. By double uncompromisingness if agent $i$ moves her peak from $p^{\prime i}$ to $p^{i}$, the location $F^{A}(R)$ does not change from $F^{A}\left(R^{\prime}\right)$. But this contradicts our assumption of $F^{A}(R) \neq$ $F^{A}\left(R^{\prime}\right)$. Supposing $F_{1}^{A}\left(R^{\prime}\right)>\max \left(F_{1}^{A}(R), p_{1}^{\prime i}\right)$, leads to a similar contradiction.

2. Proof for $F^{B}\left(R^{\prime}\right)$ is similar.

For the next Lemma B.2 we assume that $F^{B}(R) \in \bar{Q}_{++}\left(\frac{F^{A}(R)+F^{B}(R)}{2}\right)$. We can do this without loss of generality.

Lemma 3.2. Suppose $F$ is a double uncompromising, strategy-proof, peaks only rule and $R, R^{\prime} \in \mathscr{R}^{n}$ are two profiles with $R^{j}=R^{\prime j}$ for all $j \in N \backslash\{i\}$, and $p, p^{\prime}$ are the respective profiles of peaks. Let, agent $i$ 's peak be such that $p^{i} \notin Q_{++}\left(\frac{F^{A}(R)+F^{B}(R)}{2}\right), p_{1}^{i}=F_{1}^{A}(R)$, and $p_{1}^{\prime i}>p_{1}^{i}$ with $p_{2}^{\prime i}=p_{2}^{i}$. Then $F_{2}^{A}\left(R^{\prime}\right)=F_{2}^{A}(R)$.

Proof. We split the proof in 3 cases depending on the relative position of $p^{\prime i}$ with respect to $\frac{F^{A}(R)+F^{B}(R)}{2}$. 


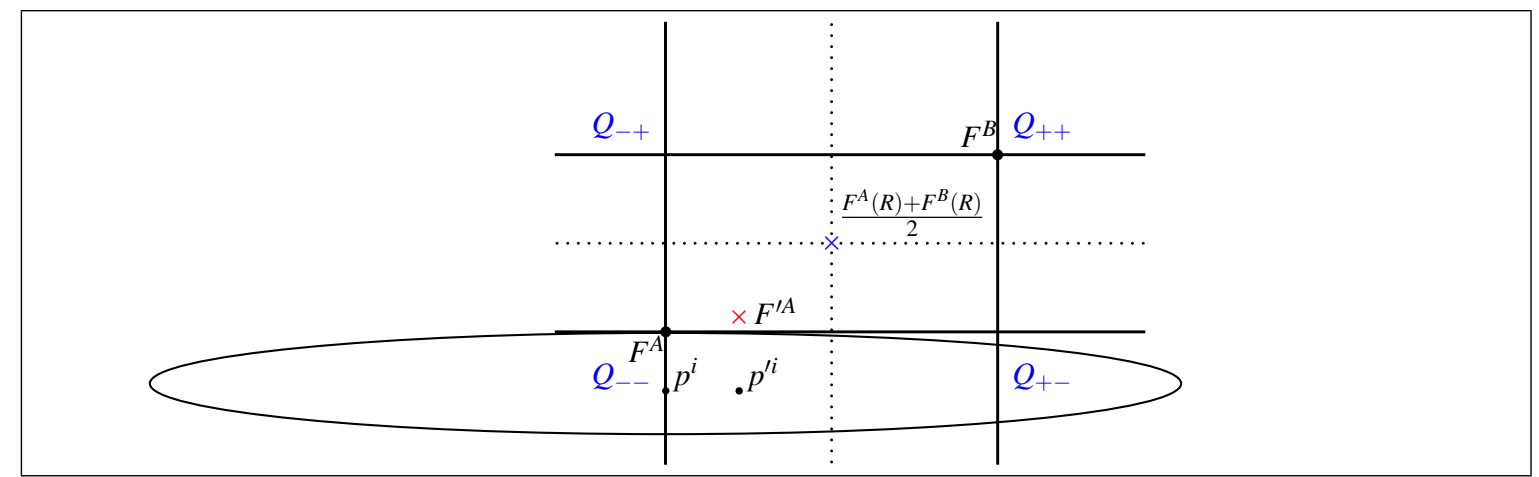

Figure 3.3: Suitable Elliptical Indifference Curve

Case 3.1. $p^{i} \in Q_{--}\left(\frac{F^{A}(R)+F^{B}(R)}{2}\right)$

Since, $p^{i} \in Q_{--}\left(\frac{F^{A}(R)+F^{B}(R)}{2}\right), F^{A}(R) P^{i} F^{B}(R)$ for any quadratic separable preference with peak at $p^{i}$.

Suppose, for the sake of contradiction, that $F_{2}^{A}\left(R^{\prime}\right)>F_{2}^{A}(R)$. Due to quadratically separable preferences of agent $i$, there exists a preference $R^{* i}=\left(p^{\prime i}, \alpha_{1}^{* i}, \alpha_{2}^{* i}\right)$ such that $F^{A}(R) P^{* i} F^{A}\left(R^{* i}, R^{-i}\right)$, which contradicts strategy-proofness. Note, that by peaksonlyness we have $F^{A}\left(R^{* i}, R^{-i}\right)=F^{A}\left(R^{\prime}\right)$. The choice of the above mentioned $\alpha^{* i}$ is given by:

$$
\begin{aligned}
& -\sqrt{\alpha_{1}^{* i}\left(F_{1}^{A}(R)-p_{1}^{\prime i}\right)^{2}+\alpha_{2}^{* i}\left(F_{2}^{A}(R)-p_{2}^{\prime i}\right)^{2}} \\
& >-\sqrt{\alpha_{1}^{* i}\left(F_{1}^{A}\left(R^{* i}, R^{-i}\right)-p_{1}^{\prime i}\right)^{2}+\alpha_{2}^{* i}\left(F_{2}^{A}\left(R^{* i}, R^{-i}\right)-p_{2}^{\prime i}\right)^{2}}
\end{aligned}
$$

or, equivalently

$$
\begin{aligned}
& \alpha_{1}^{* i}\left[\left(p_{1}^{i}-p_{1}^{\prime i}\right)^{2}-\left(F_{1}^{A}\left(R^{* i}, R^{-i}\right)-p_{1}^{\prime i}\right)^{2}\right] \\
< & \alpha_{2}^{* i}\left[\left(F_{2}^{A}\left(R^{* i}, R^{-i}\right)-p_{2}^{\prime i}\right)^{2}-\left(F_{2}^{A}(R)-p_{2}^{\prime i}\right)^{2}\right] .
\end{aligned}
$$

By assumption, $F_{2}^{A}(R)-p_{2}^{\prime i}<F_{2}^{A}\left(R^{* i}, R^{-i}\right)-p_{2}^{\prime i}$. From Lemma B.], we have, $F_{1}^{A}(R) \leqslant$ $F_{1}^{A}\left(R^{* i}, R^{-i}\right) \leqslant p_{1}^{\prime i}$. 
If $F_{1}^{A}\left(R^{* i}, R^{-i}\right)=F_{1}^{A}(R)=p_{1}^{i}$, then the above inequality is trivially satisfied and else, we need $\alpha^{* i}$ satisfying

$$
\frac{\alpha_{1}^{* i}}{\alpha_{2}^{* i}}<\frac{\left[\left(F_{2}^{A}\left(R^{* i}, R^{-i}\right)-p_{2}^{i}\right)^{2}-\left(F_{2}^{A}(R)-p_{2}^{i}\right)^{2}\right]}{\left[\left(p_{1}^{i}-p_{1}^{i}\right)^{2}-\left(F_{1}^{A}\left(R^{* i}, R^{-i}\right)-p_{1}^{\prime i}\right)^{2}\right]}
$$

where the right hand side is a well defined positive real number.

Similarly, if $F_{2}^{A}\left(R^{* i}, R^{-i}\right)<F_{2}^{A}(R)$ assumed, then it can be shown that there exists a preference $R^{\prime \prime i}=\left(p^{i} ; \alpha_{1}^{\prime \prime i}, \alpha_{2}^{\prime \prime i}\right)$ such that $F^{A}\left(R^{\prime i}, R^{-i}\right) P^{\prime \prime i} F^{A}(R)$. In combination with peaksonlyness this violates strategy-proofness. Hence, we must have $F_{2}^{A}\left(R^{\prime}\right)=F_{2}^{A}(R)$.

Case 3.2. $p^{i} \in Q_{-+}\left(\frac{F^{A}(R)+F^{B}(R)}{2}\right)$

For $p^{i} \in Q_{-+}\left(\frac{F^{A}(R)+F^{B}(R)}{2}\right)$, there exists a preference $\hat{R}^{i}=\left(p^{i} ; \hat{\alpha}_{1}^{i}, \hat{\alpha}_{2}^{i}\right)$ (with $\left.\hat{\alpha}_{1}^{i}>>\hat{\alpha}_{2}^{i}\right)$ so that, $F^{A}(R) \hat{R}^{i} F^{B}(R)$. Due to peaks-onlyness $F\left(\hat{R}^{i}, R^{-i}\right)=F(R)$. Thus we can continue similarly as Case B.Il.

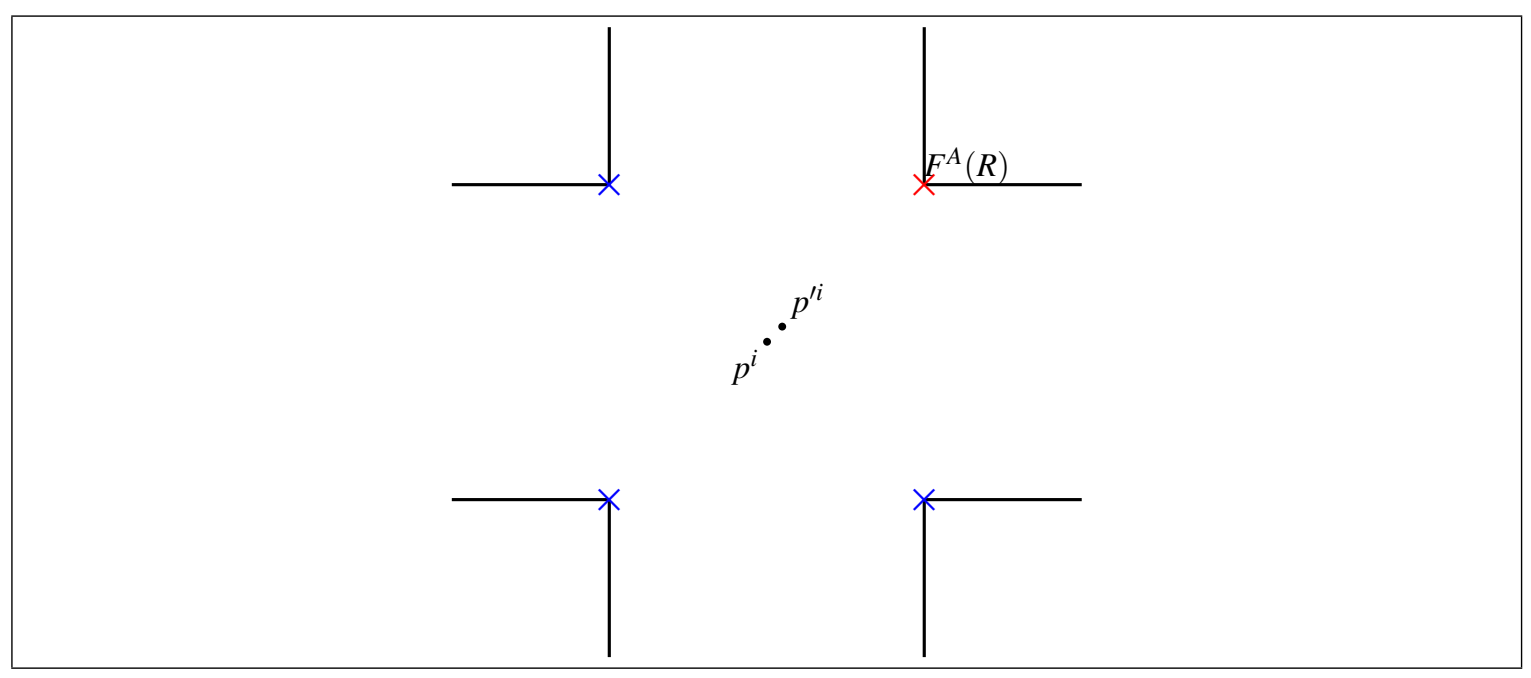

Figure 3.4: Remark B.]

Remark 3.1. Suppose $F(R)$ is the outcome for a preference profile $R$ and $R^{i}=\left(p^{i} ; \alpha_{1}^{i}, \alpha_{2}^{i}\right)$. Without loss of generality assume that $F^{A}(R) R^{i} F^{B}(R)$. Note that for any $\alpha_{1}^{i}$ and $\alpha_{2}^{i}$, 
there are 3 points which are indifferent to $F^{A}(R)$. These points are the vertices of the rectangle with center at $p^{i}$ and a vertex at $F^{A}(R)$. In Figure 3.4 they are marked with blue crosses. Take another preference $R^{\prime i}=\left(p^{\prime i} ; \alpha_{1}^{\prime i}, \alpha_{2}^{\prime i}\right)$, where $p^{\prime i}$ is close to $p^{i}$. Then due to continuity $F^{A}\left(R^{\prime i}, R^{-i}\right)$ cannot jump to those 3 indifferent points. Hence, $F^{A}\left(R^{\prime i}, R^{-i}\right) \in \bar{Q}_{++}\left(F^{A}(R)\right)$. Due to strategy-proofness $F^{A}\left(R^{\prime i}, R^{-i}\right)=F^{A}(R)$.

Lemma 3.3. Double uncompromisingness implies peaks onlyness.

Proof. We need to show that for any two preference profiles $R, R^{\prime} \in \mathscr{R}^{n}, F(R)=F\left(R^{\prime}\right)$, provided that the corresponding profiles of peaks are the same, i.e., $p^{i}=p^{i}$ for all $i \in N$.

Case 3.1. If none of the peaks are on $F^{A}(R)$ or $F^{B}(R)$, then double uncompromisingness directly implies that the outcomes do not change as long as the peaks are unchanged.

Case 3.2. If there is a peak $p^{i}$ on the outcome $F^{A}(R)$ (and)or $F^{B}(R)$, then a change of outcome without changing the peak means that agent $i$ has changed her preference. Suppose, $p^{i}=F^{A}(R)$ where $R^{i}=\left(p^{i}, \alpha_{1}^{i}, \alpha_{2}^{i}\right)$ and $R^{\prime i}=\left(p^{i}, \alpha_{1}^{\prime i}, \alpha_{2}^{\prime i}\right)$ is a preference such that $p^{i} \neq F^{A}\left(R^{\prime i}, R^{-i}\right)$. Without loss of generality, assume $p^{i} \in Q_{++}\left(F^{A}\left(R^{\prime i}, R^{-i}\right)\right)$. Due to double uncompromisingness, a change from $R^{i}$ to $R^{\prime i}$ doesn't change the outcome $F^{A}$. Therefore, we have $F^{A}\left(R^{\prime i}, R^{-i}\right)=F^{A}(R)$, which contradicts the assumption.

Thus double uncompromisingness implies peaks-onlyness.

Lemma 3.4. Unanimity and double uncompromisingness imply box property.

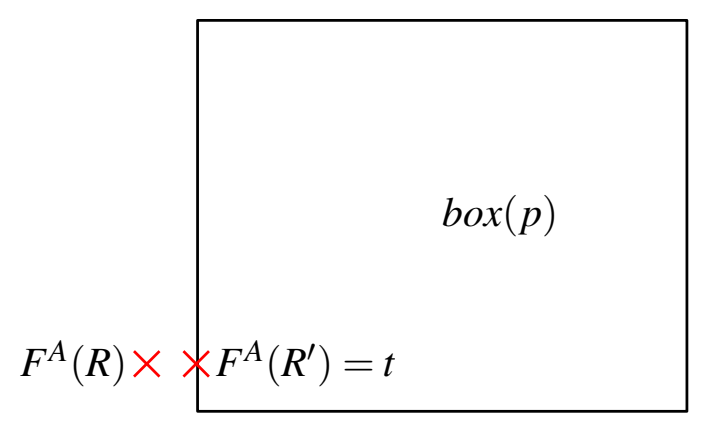

Figure 3.5: Proof of Lemma 3.4

Proof. Let $F(R)=\left(F^{A}(R), F^{B}(R)\right)$ be the outcome at preference profile $R \in \mathscr{R}^{n}$ with peaks profile $p$. To the contrary, suppose one of the locations (say, $F^{A}(R)$ ) is not in box $(p)$. 
Let $t \in \operatorname{box}(p)$ be the closest point on $\operatorname{box}(p)$ from $F^{A}(R)$. Consider a preference profile $R^{\prime}$ where all the peaks are at $t$. Due to double uncompromisingness $F^{A}\left(R^{\prime}\right)=F^{A}(R)$. But due to unanimity $F^{A}\left(R^{\prime}\right)=t$. This contradicts our assumption, which completes the proof.

Lemma 3.5. Maskin monotonicity, peaks-onlyness, continuity and strategy-proofness imply double uncompromisingness.

Proof. Suppose $F(R)$ is the outcome at preference profile $R$ with peaks profile $p$. Without loss of generality take a peak $p^{i} \in Q_{--}\left(F^{A}(R)\right)$. Suppose agent $i$ deviates and reports $p^{\prime i} \in Q_{--}\left(F^{A}(R)\right)$ and some $\alpha^{\prime i}$. Consider the following two cases.

Case 3.1. $p^{\prime i} \in Q_{++}\left(p^{i}\right) \cap Q_{--}\left(F^{A}(R)\right)$.

According to preference $R^{\prime i}=\left(p^{\prime i}, \alpha^{\prime i}\right)$, the region $U\left(F^{A}(R) \mid p^{\prime i}, \alpha^{\prime i}\right)$ is enclosed by an ellipse with center at $p^{\prime i}$ and passing through $F^{A}(R)$. If we show that there exists $\alpha^{* i}$ such that $U\left(F^{A}(R) \mid p^{\prime i}, \alpha^{\prime i}\right) \subseteq U\left(F^{A}(R) \mid p^{i}, \alpha^{* i}\right)$, then Maskin monotonicity requires that $F^{A}(R)$ is also chosen at preference profile $R^{\prime}$. Due to peaks onlyness $F(R)=F\left(R^{* i}, R^{-i}\right)$. Now, the choice of such an $\alpha^{* i}$ is given by $\frac{\alpha_{1}^{* i}}{\alpha_{2}^{* i}}=\frac{\alpha_{1}^{\prime i}}{\alpha_{2}^{\prime i}} \frac{F_{1}^{A}(R)-p_{1}^{i i}}{F_{1}^{A}(R)-p_{1}^{i}(R)-p_{2}^{i i}} \frac{F_{2}^{A}(R)-p_{2}^{i}}{\text {. This }}$ This condition for $\alpha^{* i}$ is obtained from the fact that boundaries (ellipses) of $U\left(F^{A}(R) \mid p^{\prime i}, \alpha^{\prime i}\right)$ and $U\left(F^{A}(R) \mid p^{i}, \alpha^{* i}\right)$ have a common tangent at $F^{A}(R)$. It can be shown ${ }^{\square}$ that $U\left(F^{A}(R) \mid p^{\prime i}, \alpha^{\prime i}\right) \subseteq U\left(F^{A}(R) \mid p^{i}, \alpha^{* i}\right)$. Therefore, due to Maskin monotonicity we have $F\left(R^{\prime i}, R^{-i}\right)=F(R)$.

Case 3.2. $p^{\prime i} \in Q_{--}\left(p^{i}\right)$.

To the contrary assume, that $F^{A}\left(R^{\prime i}, R^{-i}\right) \neq F(R)$, where $R^{\prime i}=\left(p^{\prime i} ; \alpha^{\prime i}\right)$. Suppose $\mid p^{i}-$ $p^{\prime i} \mid \leqslant \varepsilon$ for some $\varepsilon>0$. Due to continuity there exists $\varepsilon$ such that $F^{A}\left(R^{\prime i}, R^{-i}\right) \in Q_{++}\left(p^{i}\right)$. Then we can apply case B.]: with original preference $R^{\prime i}$ and deviation to $R^{i}$, which implies $F^{A}(R)=F^{A}\left(R^{\prime i}, R^{-i}\right)$, contradicting our assumption.

Rest of this section shows combined implication of all the conditions on a rule. Because of peaks-onlyness often (when it doesn't create confusion) we write $F(p)$ as a function of only profile of peaks. The next Proposition 3.1 is an important result in this section showing independence of two coordinates of a rule.

\footnotetext{
${ }^{2}$ Though the intuition is simple, the proof consists of solving polynomials with degree four, so we don't include that in the thesis.
} 
Proposition 3.1. Suppose F satisfies Maskin monotonicity, strategy-proofness, peaksonlyness, continuity and box property. Take any peaks profile $p \in \mathbf{R}^{2 \times n}$. For an agent $i \in N$ if $p^{\prime i}$ is such that $p_{2}^{i}=p_{2}^{\prime i}$ and $p_{1}^{\prime i}>p_{1}^{i}$, then

$$
\begin{aligned}
& \text { 1. } F_{2}^{A}\left(p^{\prime i}, p^{-i}\right)=F_{2}^{A}(p) \text { and } F_{2}^{B}\left(p^{\prime i}, p^{-i}\right)=F_{2}^{B}(p) \text {. } \\
& \text { 2. } F_{1}^{A}(p) \leqslant F_{1}^{A}\left(p^{\prime i}, p^{-i}\right) \leqslant p_{1}^{\prime i} \text { and } F_{1}^{B}(p) \leqslant F_{1}^{B}\left(p^{\prime i}, p^{-i}\right) \leqslant p_{1}^{\prime i} \text {. }
\end{aligned}
$$

To prove the proposition we need the following Lemmas 3.6 , B.7, 3.8 based on 3 possible locations of $F^{A}(p)$ relative to $F^{B}(p)$.

The first situation is when $F^{A}(p)$ and $F^{B}(p)$ are different in both $X$ and $Y$ coordinates.

Lemma 3.6. Proposition $[$.$] is true if F_{1}^{A}(p) \neq F_{1}^{B}(p)$ and $F_{2}^{A}(p) \neq F_{2}^{B}(p)$.

Proof. Without loss of generality assume $F^{A}(p) \in Q_{--}\left(F^{B}(p)\right)$. We partition $\mathbf{R}^{2}$ as follows:

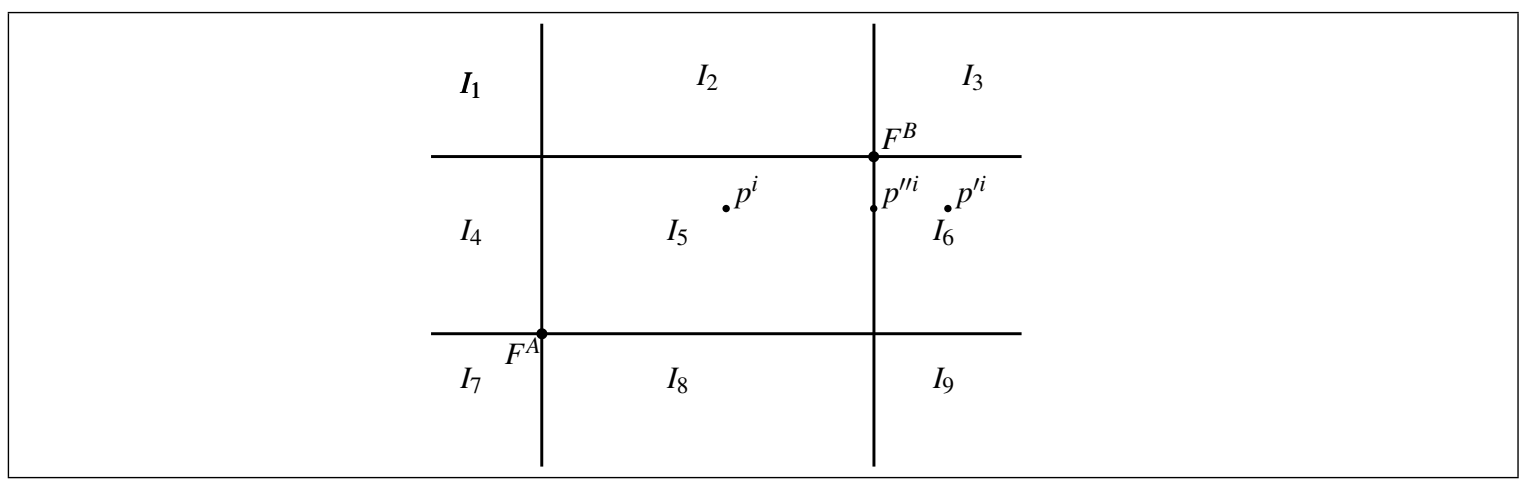

Figure 3.6: Partition of $\mathbf{R}^{2}$ based on $F^{A}$ and $F^{B}$.

As long as $p^{\prime i} \notin Q_{++}\left(\frac{F^{A}(p)+F^{B}(p)}{2}\right)$, the result simply follows from Lemma B.] and B.2. For $p^{i} \in Q_{++}\left(\frac{F^{A}(p)+F^{B}(p)}{2}\right), F^{B}(p) R^{\prime i} F^{A}(p)$ for any $R^{\prime i}=\left(p^{\prime i}, \alpha^{\prime i}\right)$. We are done by simply applying Lemma B.2, reversing the role of $F^{A}($.$) and F^{B}($.$) .$

Next we consider the situation when $F^{A}(p)$ and $F^{B}(p)$ are different but have the same $X$-coordinate.

Lemma 3.7. Proposition 1.$]$ is true if $F_{1}^{A}(p)=F_{1}^{B}(p)$ but $F_{2}^{A}(p) \neq F_{2}^{B}(p)$. 
Proof. Without loss of generality assume $F_{2}^{A}(p)<F_{2}^{B}(p)$. We also assume $p_{2}^{i} \leqslant$ $\frac{F_{2}^{A}(p)+F_{2}^{B}(p)}{2}$, which means that $F^{A} R^{i} F^{B}$ for all $R^{i} \in \mathscr{R}$ with peak $p^{i}$.

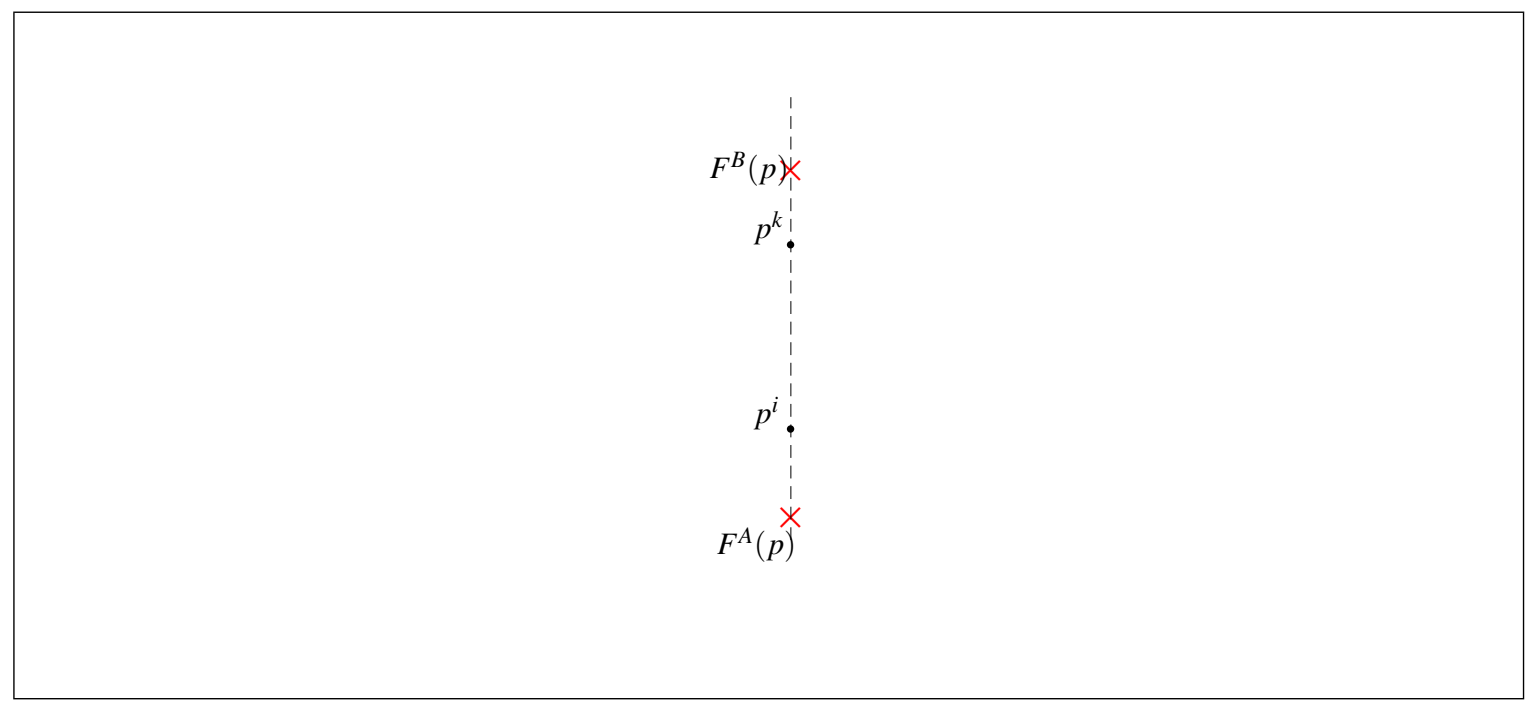

Figure 3.7: Proof of Lemma 3.7

If moving from $p^{i}$ to $p^{\prime i}$ doesn't effect $F^{A}(p)$, then due to lexmax preferences and strategyproofness $F^{B}(p)$ cannot move either. So, we consider the case when $F^{A}(p)$ is effected by this movement.

Due to box property there is at least one agent who has peak $p^{k}$ with $p_{2}^{k}>\frac{F_{2}^{A}(p)+F_{2}^{B}(p)}{2}$. We move $p^{i}$ and $p^{k}$ on straight line $x=F_{1}^{A}(p)$. By double uncompromisingness outcomes do not change. Using Lemma 3.2 we have $F_{1}^{A}(p) \leqslant F_{1}^{A}\left(p^{\prime i}, p^{-i}\right) \leqslant p_{1}^{\prime i}$ and $F_{2}^{A}\left(p^{\prime i}, p^{-i}\right)=$ $F_{2}^{A}(p)$. By double uncompromisingness $F^{B}\left(p^{\prime i}, p^{-i}\right)$ satisfies $F_{1}^{B}\left(p^{\prime i}, p^{-i}\right) \geqslant F_{1}^{B}(p)$. Suppose, for the sake of contradiction, that $F_{2}^{B}\left(p^{\prime i}, p^{-i}\right)>F_{2}^{B}(p)$. The situation is depicted below in one of the two situations in Figure 3.8. 

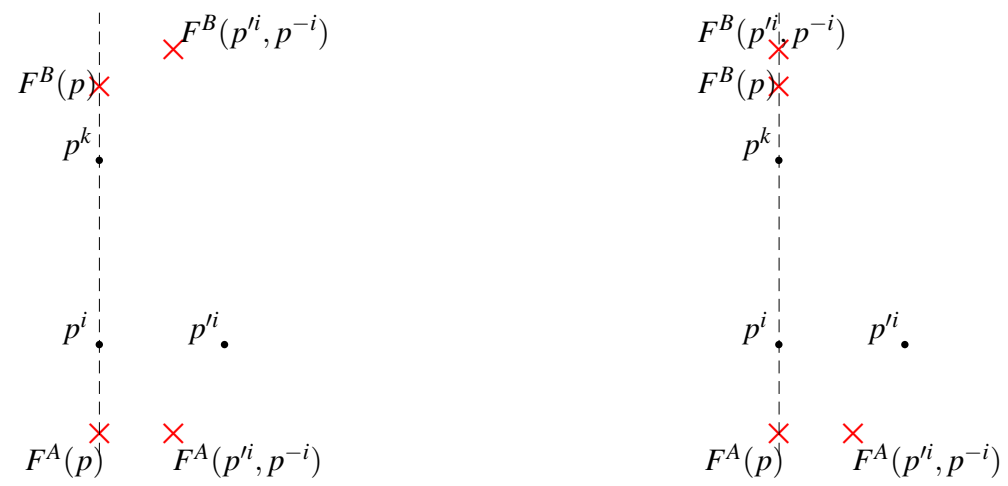

Figure 3.8: Proof of Lemma 3.7

Consider the profile $\left(p^{\prime i}, p^{\prime k}, p^{-i,-k}\right)$, where $p^{\prime k}$ is such that $p_{2}^{\prime k}=p_{2}^{k}$ and $p_{1}^{\prime k}=p_{1}^{k}-\varepsilon$. Due to double uncompromisingness $F^{A}\left(p^{\prime i}, p^{\prime k}, p^{-i,-k}\right)=F^{A}\left(p^{\prime i}, p^{-i}\right)$. By Lemma B.2, we have $F_{1}^{B}\left(p^{\prime i}, p^{-i}\right) \geqslant F_{1}^{B}\left(p^{\prime i}, p^{\prime k}, p^{-i,-k}\right) \geqslant p_{1}^{\prime k}$ and $F_{2}^{B}\left(p^{\prime i}, p^{\prime k}, p^{-i,-k}\right)=F_{2}^{B}\left(p^{\prime i}, p^{-i}\right)$. Thus we have a scenario like Figure 3.9.
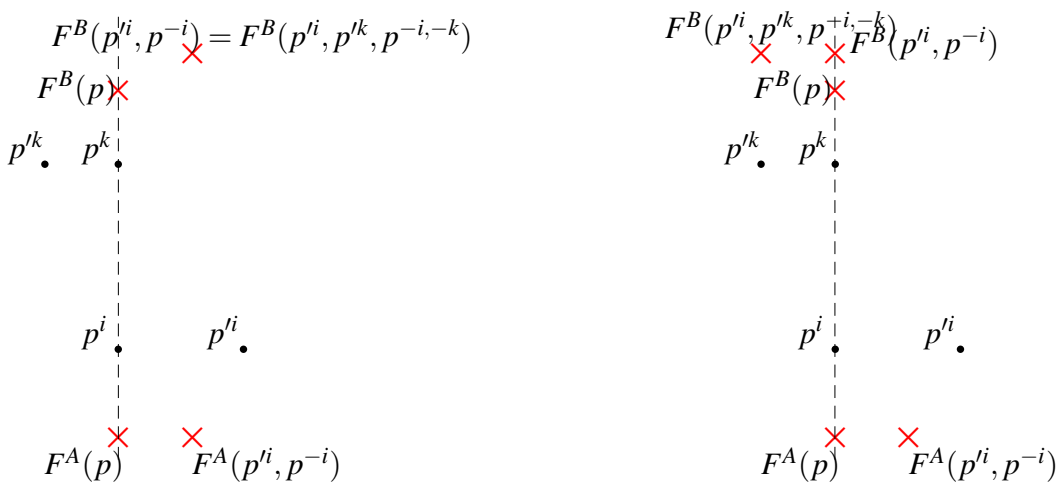

Figure 3.9: Proof of Lemma B.7

So, basically $F_{2}^{A}\left(p^{\prime i}, p^{\prime k}, p^{-i,-k}\right)=F_{2}^{A}(p)$ and $F_{2}^{B}\left(p^{\prime i}, p^{\prime k}, p^{-i,-k}\right)>F_{2}^{B}(p)$.

Now reverse the order of moving, i.e., first move $p^{k}$ to $p^{\prime k}$ and then move $p^{i}$ to $p^{\prime i}$. By 
similar reasoning $F_{2}^{B}\left(p^{\prime i}, p^{\prime k}, p^{-i,-k}\right)=F_{2}^{B}(p)$ and $F_{2}^{A}\left(p^{\prime i}, p^{\prime k}, p^{-i,-k}\right)>F_{2}^{A}(p)$. This essentially leads to a contradiction.

Next we consider the situation when $F^{A}(p)$ and $F^{B}(p)$ have the same $Y$-coordinate but their $X$-coordinates are different.

Lemma 3.8. Proposition $[$.$] is true if F_{1}^{A}(p) \neq F_{1}^{B}(p)$ but $F_{2}^{A}(p)=F_{2}^{B}(p)$.

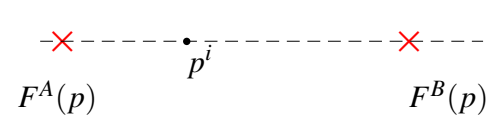

Figure 3.10: Proof of Lemma B.8

Proof. Without loss of generality assume $F_{1}^{A}(p)<F_{1}^{B}(p)$. Because of double uncompromisngness we only need to consider the cases where $p_{1}^{i} \leqslant F_{1}^{B}(p)$ and $p_{1}^{\prime i} \geqslant F_{1}^{A}(p)$.

Case 3.1. $p_{1}^{\prime i} \leqslant F_{1}^{B}(p)$

Since $p^{i}$ and $p^{\prime i}$ differ only in $X$-axis, the movement from $p^{i}$ to $p^{\prime i}$ does not effect $F^{B}(p)$ as long as $p_{1}^{\prime i} \leqslant F_{1}^{B}(p)$. Take $p_{1}^{i}=F_{1}^{A}(p)$. From Lemma 3.$]$ we have $F^{A}\left(p^{\prime}\right)=F^{A}(p)$.

Case 3.2. $p_{1}^{\prime i}>F_{1}^{B}(p)$

Applying Lemma B.1 this is straight forward.

This situation considers having both $F^{A}(p)$ and $F^{B}(p)$ at the same spot.

Lemma 3.9. Proposition 3.7 is true if $F^{A}(p)=F^{B}(p)$.

Proof. By double uncompromisingness, as long as $p^{i}, p^{\prime i}$ are on the same quadrant with respect to $F^{A}(p)$, the locations remain the same. Essentially, we need to consider cases when $p^{i}=F^{A}(p)=F^{B}(p)$ and $p^{\prime i}$ is such that $p_{1}^{\prime i}>p_{1}^{i}$ and $p_{2}^{\prime i}=p_{2}^{\prime i}$. 


$$
\begin{gathered}
p^{*} \\
p^{i}=F^{A}(p)=F^{B}(p)
\end{gathered}
$$

Figure 3.11: Proof of Lemma 3.9

If both $F_{2}^{A}\left(p^{*}, p^{-i}\right) \neq F_{2}^{A}(p)$ and $F_{2}^{B}\left(p^{*}, p^{-i}\right) \neq F_{2}^{B}(p)$, then for agent $i$ there exists a preference $R^{* i}=\left(p^{* i}, \alpha^{* i}\right)$ (with $\left.\alpha_{2}^{* i}>>\alpha_{1}^{* i}\right)$ such that $F(p) P^{* i} F\left(p^{*}, p^{-i}\right.$ ), which violates strategy proofness. But if one of the locations move on the same $Y-$ coordinate as $p_{2}^{i}$ then we need a longer argument as follows:

$$
\begin{aligned}
& F^{F^{B}}\left(p^{*}, p^{-i}\right)
\end{aligned}
$$

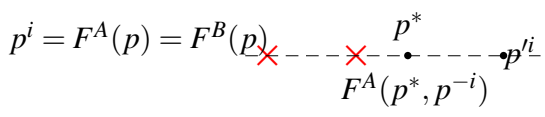

Figure 3.12: Proof of Lemma 3.9

$$
\begin{aligned}
& F^{B}\left(p^{*}, p^{-i}\right) \\
& p^{i}=F^{A}(p)=F^{B}(\underline{p})_{\star} \begin{array}{c}
F^{A}\left(p^{*}, p^{-i}\right) \\
p^{*}-\cdots-\cdots
\end{array}
\end{aligned}
$$

Figure 3.13: Proof of Lemma 3.9

Without loss of generality assume $F_{2}^{A}\left(p^{*}, p^{-i}\right)=F_{2}^{A}(p)$, and $F_{2}^{B}\left(p^{*}, p^{-i}\right)>F_{2}^{B}(p)$. Due to double uncompromisingness we have $F_{1}^{A}(p) \leqslant F_{1}^{A}\left(p^{*}, p^{-i}\right) \leqslant p_{1}^{*}$ and $F_{1}^{B}(p) \leqslant$ $F_{1}^{B}\left(p^{*}, p^{-i}\right) \leqslant p_{1}^{*}$. If we move back to $F^{A}\left(p^{*}, p^{-i}\right)$ from $p^{*}$, then $F^{B}\left(p^{*}, p^{-i}\right)$ must also go back horizontally to $\left(F_{1}^{A}\left(p^{*}, p^{-i}\right), F_{2}^{B}\left(p^{*}, p^{-i}\right)\right)$. At this step we refer to Lemma 3.7, and move the peak of agent $i$ to $p$. This leads to $F_{2}^{B}(p)=F_{2}^{B}\left(p^{*}, p^{-i}\right)$. This is a contradiction with the outcome at the original profile $p$. 


\section{Proof of the Proposition}

As all possible relative locations of $F^{A}(R)$ and $F^{B}(R)$ are covered in Lemmas 3.6, 3.7, [3.8, combining them completes the proof of the Proposition B.].

Corollary 3.1. Suppose F satisfies strategy-proof, Maskin monotonicity, continuity, peaksonlyness and box property. Take any peaks profile $p \in \mathbf{R}^{2 \times n}$. For an agent $i \in N$ if $p^{\prime i}$ is such that $p_{1}^{i}=p_{1}^{\prime i}$ and $p_{2}^{\prime i}>p_{2}^{i}$, then

$$
\begin{aligned}
& \text { 1. } F_{1}^{A}\left(p^{\prime i}, p^{-i}\right)=F_{1}^{A}(p) \text { and } F_{2}^{A}(p) \leqslant F_{2}^{A}\left(p^{\prime i}, p^{-i}\right) \leqslant p_{2}^{\prime i} \text { and } \\
& \text { 2. } F_{1}^{B}\left(p^{\prime i}, p^{-i}\right)=F_{1}^{B}(p) \text { and } F_{2}^{B}(p) \leqslant F_{2}^{B}\left(p^{\prime}, p^{-i}\right) \leqslant p_{2}^{\prime i} \text {. }
\end{aligned}
$$

Proposition B.] and Corollary 3.1 together with intermediate strategy-proofness ${ }^{[1]}$ shows that a rule is coordinatewise, i.e., $F$ can be written as two independent functions one for each coordinate, for example,

$$
\begin{aligned}
F(p) & =\left(F_{1}\left(p_{1}^{1}, \ldots, p_{1}^{n}\right), F_{2}\left(p_{2}^{1}, \ldots, p_{2}^{n}\right)\right) \\
& =\left(\left(F_{1}^{A}\left(p_{1}^{1}, \ldots, p_{1}^{n}\right), F_{1}^{B}\left(p_{1}^{1}, \ldots, p_{1}^{n}\right)\right),\left(F_{2}^{A}\left(p_{2}^{1}, \ldots, p_{2}^{n}\right), F_{2}^{B}\left(p_{2}^{1}, \ldots, p_{2}^{n}\right)\right)\right) .
\end{aligned}
$$

\section{Double Generalised Median Rule and Its Properties}

Suppose, there exists phantom locations over the $X$-coordinate for all $S \subseteq N$ denoted by $a_{1}(S), b_{1}(S)$ and phantom locations over the $Y$-coordinate denoted by $a_{2}(S), b_{2}(S)$. For all $S \subseteq N, a_{1}(S), a_{2}(S), b_{1}(S), b_{2}(S) \in \mathbf{R} \cup\{-\infty, \infty\}$.

Given phantom locations $a_{1}(S), a_{2}(S), b_{1}(S), b_{2}(S)$ for all $S \subseteq N$, a double generalised median rule is denoted by $V(p \mid a, b)=\left(V^{A}(p \mid a, b), V^{B}(p \mid a, b)\right)$ and defined as follows.

$$
\begin{aligned}
& V^{A}(p \mid a, b)=\left(\min _{S \subseteq N}\left[\max _{i \in S}\left\{p_{1}^{i}, a_{1}(S)\right\}\right], \min _{S \subseteq N}\left[\max _{i \in S}\left\{p_{2}^{i}, a_{2}(S)\right\}\right]\right)=\left(V_{1}^{A}, V_{2}^{A}\right), \\
& V^{B}(p \mid a, b)=\left(\min _{S \subseteq N}\left[\max _{i \in S}\left\{p_{1}^{i}, b_{1}(S)\right\}\right], \min _{S \subseteq N}\left[\max _{i \in S}\left\{p_{2}^{i}, b_{2}(S)\right\}\right]\right)=\left(V_{1}^{B}, V_{2}^{B}\right),
\end{aligned}
$$

\footnotetext{
${ }^{3}$ A rule is intermediate strategy-proof if it is not manipulable by any coalition $S$ at $R$ via $R^{\prime}$ such that $R^{i}=R^{j}$ for all $i, j \in S$. It is easy to see that strategy-proofness implies intermediate strategy-proofness.
} 
We assume for all $S \subseteq T \subseteq N, a_{1}(S) \geqslant a_{1}(T), a_{2}(S) \geqslant a_{2}(T), b_{1}(S) \geqslant b_{1}(T), b_{2}(S) \geqslant$ $b_{2}(T)$. We also assume $a_{1}(N)=b_{1}(N)=a_{2}(N)=b_{2}(N)=-\infty$ and $a_{1}(\emptyset)=b_{1}(\emptyset)=$ $a_{2}(\emptyset)=b_{2}(\emptyset)=\infty$.

For all $S, T \subseteq$ with same cardinality, if $a_{1}(S)=a_{1}(T), a_{2}(S)=a_{2}(T), b_{1}(S)=$ $b_{1}(T), b_{2}(S)=b_{2}(T)$, then $V(p \mid a, b)$ treats every agent equally. In this case, the phantom locations are basically $n+1$ points $a_{1}^{1}, \ldots, a_{1}^{n+1} \in \mathbf{R}$ where $a_{1}^{i}$ denotes the location corresponding to a coalition with cardinality $i$, with $a_{1}^{i} \geqslant a_{1}^{i+1} \cdot V_{1}^{A}(p \mid a, b)$ is computed by taking median of $\left\{p_{1}^{1}, \ldots, p_{1}^{n}, a_{1}^{1}, \ldots, a_{1}^{n+1}\right\}$. Therefore, the only way to change the chosen location is to misreport a peak which crosses coordinate(s) of the chosen location, but this can potentially move the new chosen location farther away from the original peak, but cannot move it closer. By definition of $V(p \mid a, b)$, it is peaks-only and moreover if a peak is at one side of the chosen outcome, then it cannot change the outcome by staying in that same side of the outcome. Essentially, this is why $V(p \mid a, b)$ is double uncompromising.

\section{Properties of Double Generalised Median Rule}

Lemma 3.10. $V(p \mid a, b)$ is double uncompromising.

Proof. Consider agent $i \in N$. Suppose agent $i$ unilaterally deviates from preference $R^{i}$ to $R^{\prime i}$ and $p^{i}, p^{\prime i}$ are the corresponding peaks. We have to show that as long as $p^{i}$ and $p^{\prime i}$ are in the same quadrant with respect to $V^{A}(p \mid a, b)$, the new location $V^{A}\left(p^{\prime i}, p^{-i} \mid a, b\right)$ is the same as $V^{A}(p \mid a, b)$, and the same is true for $V^{B}(p \mid a, b)$. We present this proof for $V^{A}(p \mid a, b)$. The proof for $V^{B}(p \mid a, b)$ is similar.

We consider the case when $p^{\prime i}, p^{i} \in Q_{++}\left(V^{A}(p \mid a, b)\right)$. We need to show that $O^{i}\left(V(p \mid a, b) ; R^{-i}\right)=\left\{\left(V^{A}(p \mid a, b), y\right) \mid y=V^{B}\left(p^{i}, p^{-i} \mid a, b\right)\right\}$. There are the following four possibilities

(a) $p_{1}^{\prime i} \geqslant p_{1}^{i}, p_{2}^{\prime i} \geqslant p_{2}^{i}$

(b) $p_{1}^{\prime i} \geqslant p_{1}^{i}, p_{2}^{i} \leqslant p_{2}^{i}$,

(c) $p_{1}^{\prime i} \leqslant p_{1}^{i}, p_{2}^{\prime i} \geqslant p_{2}^{i}$,

(d) $p_{1}^{\prime i} \leqslant p_{1}^{i}, p_{2}^{\prime i} \leqslant p_{2}^{i}$.

\footnotetext{
${ }^{4}$ This ensures that $V(p \mid a, b)$ satisfies the box-property.
} 
Case 3.1. (a) $p_{1}^{\prime i} \geqslant p_{1}^{i}, p_{2}^{\prime i} \geqslant p_{2}^{i}$

For any $S \subseteq N$, increasing $p_{1}^{i}$ to $p_{1}^{\prime i}$ cannot decrease the value of $\max _{j \in S}\left\{p_{1}^{j}, a_{1}(S)\right\}$ and it can only increase the value if $i \in S$. By definition of $V(p \mid a, b)$ there exists an $S^{\star} \subseteq N$ such that $V_{1}^{A}(p \mid a, b)=\max _{j \in S^{\star}}\left\{p_{1}^{j}, a_{1}\left(S^{\star}\right)\right\}$. Because $p_{1}^{i}>V_{1}^{A}(p \mid a, b)$ this implies that $i \notin S^{\star}$. Hence, $V_{1}^{A}\left(p^{\prime i}, p^{-i} \mid a, b\right)=\max _{j \in S^{\star}}\left\{p_{1}^{j}, a_{1}\left(S^{\star}\right)\right\}=V_{1}^{A}(p \mid a, b)$. Similarly $V_{2}^{A}\left(p^{\prime i}, p^{-i} \mid a, b\right)=V_{2}^{A}(p \mid a, b)$.

Case 3.2. (c) $p_{1}^{\prime i} \leqslant p_{1}^{i}, p_{2}^{\prime i} \geqslant p_{2}^{i}$,

Since $V_{1}^{A}(p \mid a, b)$ is non-decreasing in its arguments we have $V_{1}^{A}\left(p^{\prime i}, p^{-i} \mid a, b\right) \leqslant$ $V_{1}^{A}(p \mid a, b)$. Suppose, for the sake of contradiction, that $V_{1}^{A}\left(p^{\prime i}, p^{-i} \mid a, b\right)<V_{1}^{A}(p \mid a, b)$. This is only possible if there exists an $S^{\star} \subseteq N$ with $i \in S^{\star}$ such that both $V_{1}^{A}(p \mid a, b)=$ $\max _{j \in S^{\star}}\left\{p_{1}^{j}, a_{1}\left(S^{\star}\right)\right\}$ and $V_{1}^{A}(p \mid a, b)>\max \left\{p_{1}^{\prime j}, \max _{j \in S^{\star} \backslash\{i\}}\left\{p_{1}^{j}, a_{1}\left(S^{\star}\right)\right\}\right\}$. This implies $p_{1}^{i}=\max _{j \in S^{\star}}\left\{p_{1}^{j}, a_{1}\left(S^{\star}\right)\right\}=V_{1}^{A}(p \mid a, b)$, which contradicts the assumption of $p_{1}^{i}>$ $V_{1}^{A}(p \mid a, b)$. By case (a), $V_{2}^{A}\left(p^{\prime i}, p^{-i} \mid a, b\right)=V_{2}^{A}(p \mid a, b)$.

Case (b) and (d) can be argued similarly.

We started with assuming that $p^{\prime i}, p^{i} \in Q_{++}\left(V^{A}(p \mid a, b)\right)$. Similar reasoning can be applied in case the similar thing is assumed for other quadrants. Thus the proof is complete.

Lemma 3.11. $V(p \mid a, b)$ is strategy-proof.

Proof. Suppose $R \in \mathscr{R}^{n}$ and any $i \in N$. Let $p$ be the corresponding peaks profile.

Case 3.1. If $V(p \mid a, b)=\left(p^{i}, p^{i}\right)$, then agent $i$ obtains her most preferred alternative and so cannot manipulate the outcome.

Case 3.2. If $V(p \mid a, b)=\left(p^{i}, V^{B}(p \mid a, b)\right)$, then $V^{B}(p \mid a, b) \in Q . .\left(p^{i}\right)$.

Subcase 3.2.1. Consider the case when $V^{B}(p \mid a, b) \in Q_{++}\left(p^{i}\right)$. By definition of $V^{B}(p \mid a, b)$, as long as $p^{\prime i} \in Q_{--}\left(V^{B}(p \mid a, b)\right)$, the option set for agent $i$, i.e. $O^{i}\left(V(p \mid a, b) ;\left(p^{-i}\right)\right)=$ $\left\{\left(x, V^{B}(p \mid a, b)\right) \mid x=V^{A}\left(p^{\prime i}, p^{-i} \mid a, b\right)\right.$, for all $\left.p^{\prime i} \in \mathbf{R}^{2}\right\}$. If $p^{\prime i} \notin Q_{--}\left(V^{B}(p \mid a, b)\right)$, then $V(p \mid a, b) P^{i}(x, y)$ for all $(x, y) \in O^{i}\left(V(p \mid a, b) ;\left(p^{-i}\right)\right)$. Thus agent $i$ cannot change what is chosen in her favour.

Subcase 3.2.2. For $V^{B}(p \mid a, b) \in Q_{-+}\left(p^{i}\right)$. By definition of $V^{B}(p \mid a, b)$, as long as $p^{\prime i} \in Q_{+-}\left(V^{B}(p \mid a, b)\right)$, the option set for agent $i$, i.e. $O^{i}\left(V(p \mid a, b) ;\left(p^{-i}\right)\right)=$ $\left\{\left(x, V^{B}(p \mid a, b)\right) \mid x=V^{A}\left(p^{\prime i}, p^{-i} \mid a, b\right)\right.$, for all $\left.p^{\prime i} \in \mathbf{R}^{2}\right\}$. If $p^{\prime i} \notin Q_{+-}\left(V^{B}(p \mid a, b)\right)$, then $V(p \mid a, b) P^{i}(x, y)$ for all $(x, y) \in O^{i}\left(V(p \mid a, b) ;\left(p^{-i}\right)\right)$. Thus agent $i$ cannot change what is chosen in her favour. 
Subcase 3.2.3. For $V^{B}(p \mid a, b) \in Q_{--}\left(p^{i}\right)$ and $V^{B}(p \mid a, b) \in Q_{+-}\left(p^{i}\right)$ similar arguments can be used to show that manipulation is not profitable for agent $i$.

Case 3.3. The case when $V(p \mid a, b)=\left(V^{A}(p \mid a, b), p^{i}\right)$ with $V^{A}(p \mid a, b) \in Q . .\left(p^{i}\right)$ can be examined in the same way as the previous case.

Case 3.4. If $V^{A}(p \mid a, b) \neq p^{i}$ and $V^{B}(p \mid a, b) \neq p^{i}$, then we have the following subcases.

Subcase 3.4.1. If $p^{i} \in Q_{++}\left(V^{A}(p \mid a, b)\right)$ and $p^{i} \in Q_{++}\left(V^{B}(p \mid a, b)\right)$, then by using double uncompromisingness of $V(p \mid a, b)$ we have that as long as $p^{\prime i} \in Q_{++}\left(V^{A}(p \mid a, b)\right)$, the option set $O^{i}\left(V(p \mid a, b) ;\left(p^{-i}\right)\right)=\left\{\left(V^{A}(p \mid a, b), y\right) \mid y=V^{B}\left(p^{\prime i}, p^{-i} \mid a, b\right)\right.$ for all $\left.p^{\prime \in \mathbf{R}^{2}}\right\}$ and similarly as long as $p^{\prime i} \in Q_{++}\left(V^{B}(p \mid a, b)\right)$, the option set $O^{i}\left(V(p \mid a, b) ;\left(p^{-i}\right)\right)=$ $\left\{\left(x, V^{B}(p \mid a, b)\right) \mid x=V^{A}\left(p^{\prime i}, p^{-i} \mid a, b\right)\right.$ for all $\left.p^{\prime \epsilon} \mathbf{R}^{2}\right\}$. Suppose that $p^{\prime i}$ is such that $V^{A}\left(p^{\prime i}, p^{-i} \mid a, b\right)=V^{A}(p)$ but $V^{B}\left(p^{\prime i}, p^{-i} \mid a, b\right) \neq V^{B}(p \mid a, b)$. By definition, $V_{1}^{A}(p \mid a, b), V_{2}^{A}(p \| a, b)$ are all non-decreasing in their arguments $p$, hence $V(p \mid a, b) P^{i}(x, y)$ for all $(x, y) \in O^{i}\left(V(p \mid a, b) \mid a, b ;\left(p^{-i}\right)\right)=\left\{\left(V^{A}(p \mid a, b), y\right) \mid y=\right.$ $V^{B}\left(p^{\prime i}, p^{-i} \mid a, b\right)$ for all $\left.p^{\prime} \mathbf{R}^{2}\right\}$. Similar conclusion can be reached by assuming that $p^{\prime i}$ is such that $V^{B}\left(p^{\prime i}, p^{-i} \mid a, b\right)=V^{B}(p \mid a, b)$ but $V^{A}\left(p^{\prime i}, p^{-i} \mid a, b\right) \neq V^{A}(p \mid a, b)$. In case $p^{i}$ is such that $V^{A}\left(p^{\prime i}, p^{-i} \mid a, b\right) \neq V^{A}(p \mid a, b)$ and $V^{B}\left(p^{\prime i}, p^{-i} \mid a, b\right) \neq V^{B}(p \mid a, b)$, we can combine case 2 for $V^{A}(p \mid a, b)$ and $V^{B}(p \mid a, b)$ which leads to the final conclusion that agent $i$ cannot manipulate.

Subcase 3.4.2. If $p^{i} \in Q_{--}\left(V^{A}(p \mid a, b)\right)$ and $p^{i} \in Q_{--}\left(V^{B}(p \mid a, b)\right)$, then we can use the same arguments.

Subcase 3.4.3. Note that in all other situations when

1. $p^{i} \in Q_{++}\left(V^{A}(p \mid a, b)\right)$ and $p^{i} \in Q_{--}\left(V^{B}(p \mid a, b)\right)$,

2. $p^{i} \in Q_{--}\left(V^{A}(p \mid a, b)\right)$ and $p^{i} \in Q_{++}\left(V^{B}(p \mid a, b)\right)$,

3. $p^{i} \in Q_{++}\left(V^{A}(p \mid a, b)\right)$ and $p^{i} \in Q_{-+}\left(V^{B}(p \mid a, b)\right)$,

4. $p^{i} \in Q_{-+}\left(V^{A}(p \mid a, b)\right)$ and $p^{i} \in Q_{-+}\left(V^{B}(p \mid a, b)\right)$,

5. $p^{i} \in Q_{++}\left(V^{A}(p \mid a, b)\right)$ and $p^{i} \in Q_{+-}\left(V^{B}(p \mid a, b)\right)$,

6. $p^{i} \in Q_{+-}\left(V^{A}(p \mid a, b)\right)$ and $p^{i} \in Q_{++}\left(V^{B}(p \mid a, b)\right)$,

we can use the same argument.

This ends the proof. 


\section{Characterisation Result}

In this section we show that, if a rule $F$ satisfies strategy-proofness, Maskin monotonicity, continuity, peaks onlyness and box property, then $F(R)=V(p \mid a, b)$ for some $a, b$. The following Theorem B.] formalises this statement.

Theorem 3.1. F satisfies strategy-proofness, Maskin monotonicity, continuity, peaks onlyness if and only if $F(R)=V(p \mid a, b)$ for some $a, b$.

To prove this theorem we need the following Lemmas $3.12,3.13$ and 3.14 .

We use the following notations in subsequent lemmas. Let,

$$
\begin{gathered}
p_{\mid S}^{i}= \begin{cases}\frac{a}{\bar{a}} & \text { if } i \in S \\
\text { if } i \notin S\end{cases} \\
p_{\mid S}^{i}(\lambda, \mu)= \begin{cases}\lambda & \text { if } i \in S \\
\mu & \text { if } i \notin S\end{cases} \\
p_{\mid S}^{i}(\lambda)= \begin{cases}\lambda & \text { if } i \in S \\
\bar{a} & \text { if } i \notin S\end{cases} \\
p_{\mid S}^{i}(\mu)= \begin{cases}\frac{a}{\mu} & \text { if } i \in S \\
\mu & \text { if } i \notin S\end{cases}
\end{gathered}
$$

and $p_{\mid S}=\left(p_{\mid S}^{1}, \ldots, p_{\mid S}^{n}\right)$

The following Lemma 3.12 is about $V_{1}^{A}(p \mid a, b)$. We can prove the same for $V_{2}^{A}(p \mid a, b), V_{1}^{B}(p \mid a, b)$ and $V_{2}^{B}(p \mid a, b)$ as well.

Lemma 3.12. $V_{1}^{A}\left(p_{\mid S} \mid a, b\right)=a_{1}(S)$.

\footnotetext{
${ }^{5}$ Though Lemmas B.12 and B.13 use same techniques used in [Moulin, 1980] and [Weymark, 2011], we present them here for the sake of completeness.
} 
Proof. We need to consider the following situations with respect to whether there exists $p \in \mathbf{R}^{2^{n}}$ with $\underline{a}=V_{1}^{A}(p \mid a, b)$ or not and whether there exists $p \in \mathbf{R}^{2^{n}}$ with $\bar{a}=V_{1}^{A}(p \mid a, b)$ or not.

1. If both $\underline{a}, \bar{a}$ can be attained by $V_{1}^{A}(p \mid a, b)$, then for any $T \subseteq S$, because $p_{\mid S}^{i}=\underline{a}$ for all $i \in T$ and $a_{1}(T) \geqslant \underline{a}, \max _{i \in T}\left\{p_{\mid S}^{i}, a_{1}(T)\right\}=a_{1}(T)$. For any $T \nsubseteq S$, there is at least one $j \in T$ with $p_{\mid T}^{j}=\bar{a} \geqslant a_{1}(T)$, hence $\max _{i \in T}\left\{p_{\mid S}^{i}, a_{1}(T)\right\}=\bar{a}$. So, $V_{1}^{A}\left(p_{\mid S}\right)=\min _{T \subseteq N} \max _{i \in T}\left\{p_{\mid S}^{i}, a_{1}(T)\right\}=\min _{T \subseteq S} a_{1}(T)=a_{1}(S)$.

2. If $\underline{a}$ can be attained by $V_{1}^{A}(p \mid a, b)$ but not $\bar{a}$, then for all $\mu \in \rho\left(V_{1}^{A}().\right)$ any $T \subseteq S$, as $p_{\mid S}^{i}=\underline{a}$ for all $i \in T$ and $a_{1}(T) \geqslant \underline{a}, \max _{i \in T}\left\{p_{\mid S}^{i}, a_{1}(T)\right\}=a_{1}(T)$. For any $T \nsubseteq S$, as $p_{\mid S}^{i}=\mu$ for at least one $i \in T$, hence $\lim _{\mu \rightarrow \bar{a}} \max _{i \in T}\left\{p_{\mid S}^{i}(\mu), a_{1}(T)\right\}=\bar{a}$. So, $V_{1}^{A}\left(p_{\mid S}\right)=\lim _{\mu \rightarrow \bar{a}} \min _{T \subseteq N} \max _{i \in T}\left\{p_{\mid S}^{i}(\mu), a_{1}(T)\right\}=\min _{T \subseteq S} a_{1}(T)=a_{1}(S)$.

3. If $\bar{a}$ can be attained by $V_{1}^{A}(p \mid a, b)$ but not $\underline{a}$, then for all $\lambda \in \rho\left(V_{1}^{A}().\right)$ any $T \nsubseteq S$, as $p_{\mid S}^{i}=\bar{a}$ for at least one $i \in T$ and $a_{1}(T) \leqslant \bar{a}, \max _{i \in T}\left\{p_{\mid S}^{i}, a_{1}(T)\right\}=\bar{a}$. For any $T \subseteq S$, as $p_{\mid S}^{i}=\lambda$ for all $i \in T$, hence $\lim _{\lambda \rightarrow \underline{a}} \max _{i \in T}\left\{p_{\mid S}^{i}(\lambda), a_{1}(T)\right\}=a_{1}(T)$. So, $V_{1}^{A}\left(p_{\mid S}\right)=\lim _{\lambda \rightarrow \underline{a}} \min _{T \subseteq N} \max _{i \in T}\left\{p_{\mid S}^{i}(\lambda), a_{1}(T)\right\}=\min _{T \subseteq S} a_{1}(T)=a_{1}(S)$.

4. If none $\underline{a}, \bar{a}$ can be attained by $V_{1}^{A}(p \mid a, b)$, then combining case 3 and 4 we have $V_{1}^{A}\left(p_{\mid S}\right)=a_{1}(S)$.

In the next Lemma 3.13 we show by method of induction that $F=V$. The induction is on number of agents $n$. Essentially, we show that the rule $F_{1}^{A}(p)$ coincides with $V_{1}^{A}(p)$, i.e. it can be written as follows $F_{1}^{A}(p)=\min _{S \subseteq N} \max _{i \in S}\left\{p^{i}, a_{1}(S)\right\}$. It can be shown that $F_{2}^{A}(p), F_{1}^{B}(p)$ and $F_{2}^{B}(p)$ coincide with their counterpart from $V$ as well.

Lemma 3.13. $F_{1}^{A}(p)=\min _{S \subseteq N} \max _{i \in S}\left\{p^{i}, a_{1}(S)\right\}$.

Proof. For an $n$ agent profile $R$ we denote the function $F_{1}^{A}(R)$ as $f^{n}(R)$. The induction is on $n$. Suppose $n=1$. Then by the box property,

$$
f^{1}\left(R^{1}\right)=p_{1}^{1}=\min \left\{a_{1}(\emptyset), \max \left\{p_{1}^{1}, a_{1}(\{1\})\right\}\right\} .
$$


Here $a_{1}(\emptyset)=\infty$ and $a_{1}(\{1\})=-\infty$.

By assumption $F_{1}^{A}($.$) and f^{n}($.$) are peaks only, so we can use profile of peaks as inputs of$ these functions. For all $\left(R^{1}, \ldots, R^{n}\right) \in \mathscr{R}^{n}$ such that $\left(p_{1}^{1}, \ldots, p_{1}^{n}\right)=p$,

$$
f^{n+1}\left(p, p_{1}^{n+1}\right)=\min \left\{\bar{a}_{p}, \max \left\{p_{1}^{n+1}, \underline{a}_{p}\right\}\right\} \text { for all }\left(p, p_{1}^{n+1}\right) \in \mathbf{R}^{2 \times(n+1)} .
$$

For any fixed $p_{1}^{n+1} \in \mathbf{R}^{2}$

$$
f_{p_{1}^{n+1}}^{n+1}(p)=\min _{S \subseteq\{1, \ldots, n\}} \max _{i \in S}\left\{p_{1}^{i}, a_{1}(S)_{\mid p_{1}^{n+1}}\right\} \text { for all } p \in \mathbf{R}^{2 \times n}
$$

By lemma 3.12 we have,

$$
\lim _{\substack{\lambda \rightarrow a \\ \mu \rightarrow \bar{a}}} f^{n+1}\left(p_{\mid S}(\lambda, \mu), p_{1}^{n+1}\right)=a_{1}(S)_{\mid p_{1}^{n+1}} \text { for all } p_{1}^{n+1} \in \mathbf{R} \text { and for all } S \subseteq\{1, \ldots, n\}
$$

where

$$
\begin{aligned}
a_{1}(S)_{\mid p_{1}^{n+1}} & =\lim _{\substack{\lambda \rightarrow a \\
\mu \rightarrow \bar{a}}} \min \left\{\bar{a}_{\mid p_{\mid S}(\lambda, \mu)}, \max \left\{\underline{a}_{\mid p_{\mid S}(\lambda, \mu)}, p_{1}^{n+1}\right\}\right\} \\
= & \min _{\substack{\lambda \rightarrow a \\
\mu \rightarrow \overline{\bar{a}}}}\left\{\lim _{\substack{\lambda \rightarrow p_{\mid S} \\
\bar{a}_{\mid}(\lambda, \mu)}}, \max \left\{\lim _{\substack{\lambda \rightarrow a \\
\mu \rightarrow \overline{\bar{a}}}} \underline{a}_{\mid p_{\mid S}(\lambda, \mu)}, p_{1}^{n+1}\right\}\right\}
\end{aligned}
$$

Let $\underline{a}_{\mid p_{\mid S}}=\lim _{\substack{\lambda \rightarrow a \\ \mu \rightarrow \bar{a}}} \frac{a}{p_{\mid S}(\lambda, \mu)}$ and $\bar{a}_{\mid p_{\mid S}}=\lim _{\substack{\lambda \rightarrow a \\ \mu \rightarrow \bar{a}}} \bar{a}_{\mid p_{\mid S}(\lambda, \mu)}$.

So,

$$
a_{1}(S)_{\mid p_{1}^{n+1}}=\min \left\{\bar{a}_{\mid p_{\mid S}}, \max \left\{p_{1}^{n+1}, \underline{a}_{\mid p_{\mid S}}\right\}\right\} \text { for all } p_{1}^{n+1} \in \mathbf{R} \text { and } S \subseteq\{1, \ldots, n\}
$$

For all $\left(p, p_{1}^{n+1}\right) \in \mathbf{R}^{2 \times(n+1)}$,

$$
f^{n+1}=\min _{S \subseteq\{1, \ldots, n\}} \max _{i \in S}\left\{p_{1}^{i}, \min \left\{\bar{a}_{\mid p_{\mid S}}, \max \left\{p_{1}^{n+1}, \underline{a}_{\mid p_{\mid S}}\right\}\right\}\right\}
$$


Because $\max \{X, \min \{Y, Z\}\}=\min \{\max \{X, Y\}, \max \{X, Z\}\}$ it follows

$$
f^{n+1}=\min _{S \subseteq\{1, \ldots, n\}}\left[\min \left\{\max _{i \in S}\left\{p_{1}^{i}, \bar{a}_{\mid p_{\mid S}}\right\}, \max _{i \in S}\left\{p_{1}^{i}, p_{1}^{n+1}, \underline{a}_{\mid p_{\mid S}}\right\}\right\}\right]
$$

For all $S \in\{1, \ldots, n\}$ let $a_{1}(S)=\bar{a}_{|p| S}$ and $a_{1}(S \cup\{n+1\})=\underline{a}_{\mid p_{\mid S}}$. Then

$$
\begin{aligned}
f^{n+1}\left(p, p_{1}^{n+1}\right) & =\min _{S \subseteq\{1, \ldots, n\}}\left[\min \left\{\max _{i \in S}\left\{p_{1}^{i}, a_{1}(S)\right\}, \max _{i \in S \cup\{n+1\}}\left\{p_{1}^{i}, a_{1}(S \cup\{n+1\})\right\}\right\}\right] \\
& =\min _{S \subseteq\{1, \ldots, n+1\}} \max _{i \in S}\left\{p_{1}^{i}, a_{1}(S)\right\}
\end{aligned}
$$

Thus $F_{1}^{A}($.$) works in the same way function as V_{1}^{A}($.$) .$

Lemma 3.14. Box property implies that $a_{1}(N)=b_{1}(N)=a_{2}(N)=b_{2}(N)=-\infty$ and $a_{1}(\emptyset)=b_{1}(\emptyset)=a_{2}(\emptyset)=b_{2}(\emptyset)=\infty$.

Proof. To the contrary assume $a_{1}(N)>-\infty$. Consider a profile of peaks such that $a_{1}(N)>$ $\max _{i \in N} p_{1}^{i}$. Since, $a_{1}(N) \leqslant a_{1}(S)$ for all $S \subseteq N$, by definition of $V$, we have

$$
\begin{aligned}
V_{1}^{A}(p) & =\left(\min _{S \subseteq N}\left[\max _{i \in S}\left\{p_{1}^{i}, a_{1}(S)\right\}\right]\right. \\
& =\left(\min _{S \subseteq N}\left[a_{1}(S)\right]\right. \\
& =a_{1}(N) \\
& >\max _{i \in N} p_{1}^{i}
\end{aligned}
$$

This violates box property. All other equalities can be shown in the same manner.

\section{Proof of Theorem B.]}

(If part) Here we need to show that rule $V(p \mid a, b)$ is strategy-proof, Maskin monotonic, continuous, peaks only. Since, $V(p \mid a, b)$ is defined only on peaks it is obviously peaks only. Continuity is also easy to check. In Lemma B.10 we show that $V(p \mid a, b)$ is uncompromising and in Lemma $3 . \amalg$ we show that $V(p \mid a, b)$ is strategy proof. Combining them the proof for the if part is complete. 
(Only if part) Now we assume that rule $F$ is strategy-proof, Maskin monotonic, continuous, peaks only and show that $F$ can be written as $V(p \mid a, b)$. We have to provide a choice of $a, b$ satisfying the conditions in the definition of $V(p \mid a, b)$. Proposition 3.$]$ shows that two coordinates of rule $F$ are independent. Lemma 3.12 shows some facts about $a, b$ corresponding to $V(p \mid a, b)$. For the final proof of $F=V$ we use the method of induction on number of agents $n$ which is shown in Lemma 3.13. Additionally we need Lemma 3.14 to show the implication of the box property.

\section{Interaction of the Conditions}

Example 3.1. Strategy-proofness is independent of all other conditions.

We present a rule which is not strategy-proof but satisfies all other conditions. So, this serves as a counter example showing that strategy-proofness is independent of all other conditions. Consider the following rule:

$$
\begin{gathered}
F^{A}(p)= \begin{cases}\left(\min _{i} p_{1}^{i}, \max _{i} p_{2}^{i}\right) & \text { if } \max _{i} p_{2}^{i} \leqslant \min _{i} p_{1}^{i} \\
\left(\min _{i} p_{1}^{i}, \max \left(\min _{i} p_{1}^{i}, \min _{i} p_{2}^{i}\right)\right) & \text { if } \max _{i} p_{2}^{i}>\min _{i} p_{1}^{i}\end{cases} \\
F^{B}(p)=\left(\max _{i} p_{1}^{i}, \max _{i} p_{2}^{i}\right) .
\end{gathered}
$$

By definition this rule is peaks-only. Basically, outcomes of the rule depend on the relative positions of the $\operatorname{box}(p)$ with respect to the straight line $x=y$. From the following Figure 3.14 it is clear that this rule is continuous as well.

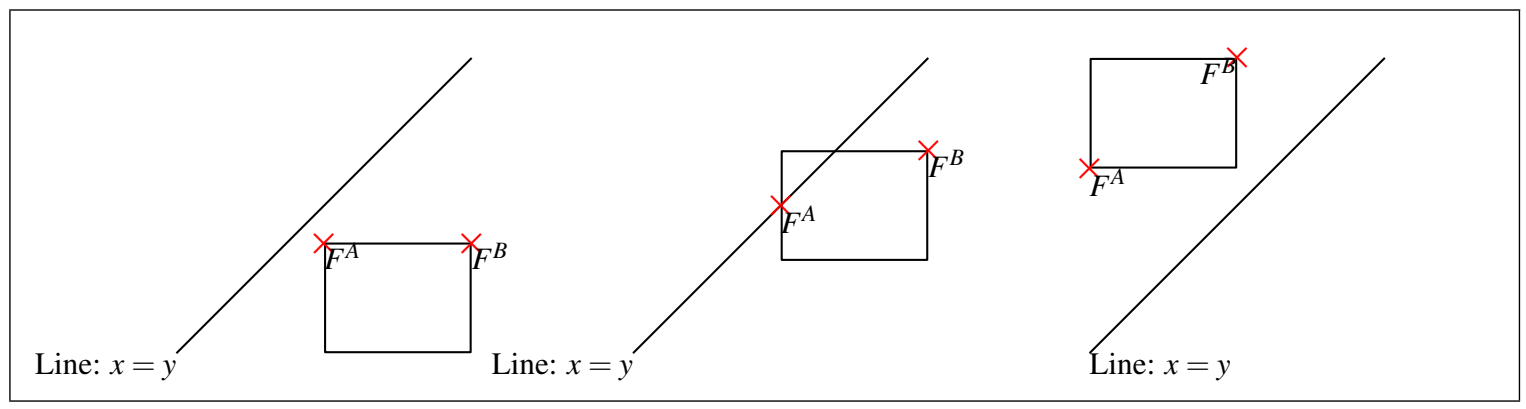

Figure 3.14: Description of the rule 
To see that it is not stratgey-proof, consider a 3 agents preference (peaks) profile: $\left(p^{1}, p^{2}, p^{3}\right)=((0,-0.75),(2,0.75)(0.5,0.5))$. Agent 1 has weights $\alpha_{1}^{1}=1$ on $X$-axis and $\alpha_{2}^{1}=0.75$ on $Y$-axis. So, the rule chooses $(0,0)$ and $(2,0.75)$ as the outcomes. Suppose, agent 1 now deviates by misreporting her peak as $p^{\prime 1}=(-0.5,-0.75)$. Now the rule chooses $(-0.5,-0.5)$.

Agent 1's utility from $(0,0)$ is $-(0.75)^{2} \alpha_{2}^{1}$ and that from $(-0.5,-0.5)$ is $-\left((0.5)^{2} \alpha_{1}^{1}+\right.$ $\left.(0.25)^{2} \alpha_{2}^{1}\right)$. So, given the values of $\alpha^{1}$ it is clear that the deviation is beneficial for agent 1. The situation is displayed in the following Figure 3.15.

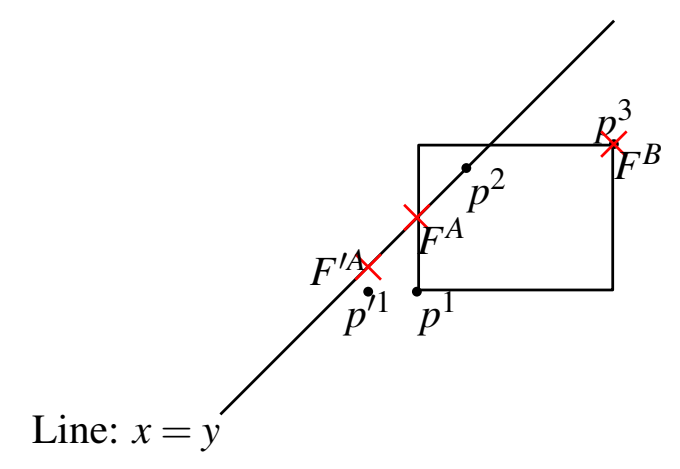

Figure 3.15: Deviation example

Example 3.2. Continuity is independent of all other conditions.

We present a rule which is not strategy-proof but satisfies all other conditions. So, this serves as a counter example showing that strategy-proofness is independent of all other conditions. Consider the following rule:

Let $c$ be the center of the $\operatorname{box}(p)$. Let $M=\left\{i \in N \mid p^{i} \in Q_{--}(c)\right\}$. If $|M|>\frac{1}{2}|N|$, then $F^{A}(p)=F^{B}(p)=\left(\min \left\{p_{1}^{i} \mid i \in N\right\}, \min \left\{p_{2}^{i} \mid i \in N\right\}\right)$. In all other cases, $F^{A}(p)=$ $\left(\min \left\{p_{1}^{i} \mid i \in N\right\}, \min \left\{p_{2}^{i} \mid i \in N\right\}\right)$ and $F^{B}(p)=\left(\max \left\{p_{1}^{i} \mid i \in N\right\}, \max \left\{p_{2}^{i} \mid i \in N\right\}\right)$.

This rule is not continuous because the change from $|M|>\frac{1}{2}|N|$ to $|M| \leqslant \frac{1}{2}|N|$ is possible even with a small change of one peak from $\left(c_{1}-\varepsilon, c_{1}-\varepsilon\right)$ to $c$. The outcomes jump due to this change, which violates continuity.

By definition, this rule satisfy the box-property.

This rule satisfies Maskin-monotonicity because of the following reason: 
Suppose $|M|>\frac{1}{2}|N|$, and as a result $F^{A}(p)=F^{B}(p)=\left(\min \left\{p_{1}^{i} \mid i \in N\right\}, \min \left\{p_{2}^{i} \mid i \in N\right\}\right)$. If agent $i$ has $p^{i} \in Q_{--}(c)$, then $U\left(F^{A}(R) ; p^{\prime i}, \alpha^{i}\right) \subseteq U\left(F^{A}(R) ; p^{i}, \alpha^{i}\right)$ implies that $p^{\prime i} \in$ $Q_{--}(c)$. Therefore, $F^{A}$ and $F^{B}$ do not change. If agent $i$ has $p^{i} \notin Q_{--}(c)$, then she cannot influence the outcome.

Suppose $|M| \leqslant \frac{1}{2}|N|$, and as a result $F^{A}(p)=\left(\min \left\{p_{1}^{i} \mid i \in N\right\}, \min \left\{p_{2}^{i} \mid i \in N\right\}\right)$ and $F^{B}(p)=\left(\max \left\{p_{1}^{i} \mid i \in N\right\}, \max \left\{p_{2}^{i} \mid i \in N\right\}\right)$. If agent $i$ 's peak $p^{i} \in Q_{--}(c)$, then she cannot influence the outcome. If agent $i$ 's peak $p^{i} \notin Q_{--}(c)$, then $U\left(F^{B}(R) ; p^{\prime i}, \alpha^{\prime i}\right) \subseteq$ $U\left(F^{B}(R) ; p^{i}, \alpha^{i}\right)$ implies $p^{i} \notin Q_{--}(c)$, hence $F^{B}$ doesn't change. Since, $F^{A}$ doesn't change at all, $U\left(F^{A}(R) ; p^{\prime i}, \alpha^{\prime i}\right) \subseteq U\left(F^{A}(R) ; p^{i}, \alpha^{i}\right)$ also implies that $F^{A}$ doesn't change.

This rule is strategy-proof because of the following reason:

Suppose $|M|>\frac{1}{2}|N|$, and as a result $F^{A}(p)=F^{B}(p)=\left(\min \left\{p_{1}^{i} \mid i \in N\right\}, \min \left\{p_{2}^{i} \mid i \in N\right\}\right)$. If agent $i$ 's peak $p^{i} \notin Q_{--}(c)$, then she cannot influence the outcome. If agent $i$ 's peak $p^{i} \in Q_{--}(c)$, then for any preference $R^{i}$ with peak at $p^{i},\left(\min \left\{p_{1}^{i} \mid i \in N\right\}, \min \left\{p_{2}^{i} \mid i \in\right.\right.$ $N\}) P^{i}\left(\max \left\{p_{1}^{i} \mid i \in N\right\}, \max \left\{p_{2}^{i} \mid i \in N\right\}\right)$. Thus unilateral deviation is not profitable.

Suppose $|M| \leqslant \frac{1}{2}|N|$, and as a result $F^{A}(p)=\left(\min \left\{p_{1}^{i} \mid i \in N\right\}, \min \left\{p_{2}^{i} \mid i \in N\right\}\right)$ and $F^{B}(p)=\left(\max \left\{p_{1}^{i} \mid i \in N\right\}, \max \left\{p_{2}^{i} \mid i \in N\right\}\right)$. If agent $i$ 's peak $p^{i} \in Q_{--}(c)$, then she cannot influence the outcome. If agent $i$ 's peak $p^{i} \notin Q_{--}(c)$, then exists a preference $R^{i}$ with peak at $p^{i}$, such that $\left(\max \left\{p_{1}^{i} \mid i \in N\right\}, \max \left\{p_{2}^{i} \mid i \in N\right\}\right) P^{i}\left(\min \left\{p_{1}^{i} \mid i \in N\right\}, \min \left\{p_{2}^{i} \mid i \in N\right\}\right)$. Thus unilateral deviation is not profitable.

Remark 3.2. From Lemma 3.3 we know that peaks onlyness is implied by other conditions and from Lemma 3.5 we know that double uncompromisingness is implied by Maskin monotonicity and other conditions. In this section Example B.] shows that strategy-proofness is not implied by double uncompromisngness and other conditions. This in turn implies that strategy-proofness is not implied if we replace double uncompromisningness by Maskin monotonicity. Example 3.2 shows that continuity is not implied by other conditions. It is easy to see that constant rule doesn't satisfy the box property. It is still an open question to check whether all the conditions in Theorem 3.17 are independent or not. 



\section{Part II}

\section{Voting Problem}



CHAPTER 4

\section{Frequency Based Analysis of Voting Rules}

\section{Introduction}

We focus on voting situations involving large numbers of voters like nation wide elections. In these voting situations voters' preferences over the candidates may vary considerably. On the other hand it is reasonable to expect that cultural backgrounds, social media, individual networks etc. induce some coherence between these voters' preferences. Coherence expressed in one or several accumulations of voters' preferences. Representing the voters' preference combinations, or profiles, at such large electorates by frequency distributions each accumulation can be seen as an agglomeration of preferences around a local mode. In that way the whole profile can be viewed as the addition or superposition of such (local) unimodal distributions yielding a multimodal distribution. In this chapter we study unimodal distributions and how reasonable outcomes of these are related to reasonable outcomes at multimodal distributions.

Taking the set of linear orders over all candidates as the set of admissible preferences a profile is a frequency distribution on this set of linear orders. This set is structured by the Kemeny distance counting the number of discordant pairs as the distance between two 
such orders. This distance is the minimal path-length needed to convert the one order into the other by swapping consecutively ordered candidates. Starting from one order at each step we arrive at a new order which has one discordant pair less with the final order than the previous order on that path. A unimodal distribution is a distribution where one order, the mode, has highest frequency and where further frequency decreases with the distance (in Kemeny sense) from the mode. Multimodal distributions are considered to be additions of such unimodal distribution. It is very reasonable to choose the mode at unimodal distribution as many well-known choice rules do so. Likewise when analysing multimodal distributions it appears natural to infer the outcome from the intersection of the local modes. This opens the possibility to study such more general distributions via unimodal ones.

We start with observing the behaviour of several well-known rules like Condorcet consistent rules, Scoring rules, Elimination rules, Kemeny-like rules, under unimodal distributions. It is worth noting that all these rules choose the mode as the outcome under unimodal distribution. We also provide a set of sufficient conditions which ensures that any rule satisfying them choose the mode at a unimodal distribution. To investigate the robustness of this result we allow for disturbances in the tail part of a unimodal distribution and show that even under this tail-perturbed distributions Condorcet consistent rules, Borda rule and Plurality rule chooses the mode as the outcome.

Intuitively, for many rules the property of choosing the mode follows because of the following. Let at the mode candidate $a$ be preferred to candidate $b$. Then a preference $R$ at which $a$ is preferred to $b$ is closer to the mode than the corresponding preference $R^{\prime}$ at which the positions of $a$ and $b$ in $R$ are swapped. Therefore $R$ has higher frequency than $R^{\prime}$. Letting $R$ run over the set of linear orders where $a$ is preferred to $b$ lets consequently $R^{\prime}$ run over the complementary set. So, in pairwise comparison $a$ will beat $b$ and the mode consists of all pairs which in pairwise comparisons beat each other: the Condorcet order. The same argument of $R$ having higher frequency than $R^{\prime}$ implies that at any score rule $a$ gets a higher score than $b$ and that $b$ is eliminated before $a$ is. So, the mode also embodies the "positional" order as well as the order of "elimination". Formalising this intuition we get some sufficient conditions. Besides anonymity and neutrality, there are two important conditions that are sufficient for this property of choosing the mode, namely, discrimination and monotonicity. Here anonymity and neutrality are used in their usual sense. Monotonicity and discrimination are formally defined in the following section. In general monotonicity properties are restrictive and therefore not satisfied by reasonable collective decision rules. The one discussed here is satisfied by many rules, for instance pairwise rules and score rules. It demands that the pair $a b$ is in the outcome at profile $q$ if it is in the outcome at profile $p$, whenever $q$ is obtained from $p$ by changing only the preference 
of some voters with a preference like $R^{\prime}$ at which $b$ is preferred to $a$ to a preference $R$ obtained by swapping the position of $a$ and $b$ in $R^{\prime}$. In this way monotonicity is defined pairwise but it is sensitive to the positions of candidates. It is therefore satisfied by many rules. Discrimination means, that a rule cannot be indifferent between two candidates, say $a$ and $b$, whenever frequency of every preference $R$ at which $a$ is preferred to $b$ out numbers frequency of $R^{\prime}$. Here $R^{\prime}$ is the preference obtained by swapping the position of $a$ and $b$ in $R$.

In the next part, for Condorcet consistent rules, Borda rule and Plurality rule we show that, all three assign the mode at the following type of perturbed unimodal distribution. Frequency declines with the distance to the mode until about half the maximal distance ${ }^{m}$. Beyond this point frequencies are free but constant at each specific distance from the mode and they are bounded above by the frequency at half the maximal distance. The combinatorial reasoning by which these results are deduced might work similarly for other collective decision rules such as other score rules or some elimination rules. But we have not found a general method to deduce this, for instance, for all score rules. The result found here indicate, however, that the mode choice of Condorcet consistent rules, Borda rule and Plurality rule are robust to considerable perturbations in the tail part of a single mode distribution. For a multimodal distribution, we show that the outcomes at each of the unimodal constituents together determine the outcome of the original distribution. For instance, for discriminating collective decision rules we show, that the outcome at the union of two unimodal distributions is between the two modes of these unimodal distributions. This result generalises to superpositions of more than two unimodal distributions. However, it is evident that at these distributions, particularly when the modes have an empty intersection, we might end up in difficult situations like Condorcet profiles. A deeper study of these situations is certainly necessary, but beyond the scope of this first exploration of unimodal distributions.

There have been some empirical work with the so-called impartial culture or uniform distribution, where each preference order is possible and occurs with equal probability. Under this assumption, the probability of a majority cycle occurring has been calculated ([see Gehrlein, 2006], [see Riker, 1982]. Many authors have noted that the impartial culture is a significant idealisation that almost certainly does not occur in real-life elections. [Grofman et al, 2003] go even further arguing that the impartial culture is a worst-case scenario in the sense that any deviation results in lower probabilities of a majority cycle ([see Grotman et al, 2006], for a complete discussion of this issue). A few other probabilistic models have been developed, for instance, Multinomial likelihood models [Gillett, 1976],

\footnotetext{
${ }^{1}$ Taking $m$ the number of candidates maximally $\left(\begin{array}{c}m \\ 2\end{array}\right)$ pairs can be discordant.
} 
[Gillett, 1978], Dual Culture [Gehrlein, 1978], Maximal Culture Condition [Fishburn and Gehrlein, 1977]. All these indicate that when voters' preferences are homogeneous, there is a increased likelihood that a Pairwise Majority Rule Winner exists meaning that Condorcet cycles are avoided. Our result go further on this as at unimodal distributions not only pairwise majority cycles are absent, but also all well-know rules have the same outcome: the mode. The main difference in our approach is that the distributions are structured by the Kemeny distance. A structure that is naturally embedded with the preferences. [Merlin et al, 2000] have studied the probability of conflicts in a U.S. presidential type of election. Their results partially resemble ours, but strictly speaking are not comparable, because of the assumption of impartial culture.

In section 4.2 we formally introduce the model. Section 4.3 shows that at unimodal distributions many collective decision rules select the mode as an outcome. In section 4.4 we formulate some sufficiency conditions for abstract collective decision rules to have the mode as an outcome at such distributions. Section 4.5 discusses disturbances in the "tail" of unimodal distributions. Section 4.6 is on multimodal distributions. A discussion in section 4.8 concludes this chapter. To make the chapter self contained we provided an appendix section 4.9 on combinatorial results needed in this chapter.

\section{Model}

We consider a finite but large set of voters $N=\{1, \ldots, n\}$, and a finite set of candidates $A=\left\{a_{1}, \ldots, a_{m}\right\}$. We assume that there are at least three candidates, hence $m \geq 3$.

Let $R$ denote a relation on $A$, i.e. $R \subseteq A \times A$. As usual for candidates $x$ and $y,(x, y) \in R$ means that at preference (relation) $R$, candidate $x$ is (weakly) ${ }^{\square}$ preferred to candidate $y$. Individual preferences of voters are formalized here by linear orders, i.e. complete, antisymmetric and transitive relations on $A$. The set of these linear orders on $A$ is denoted by $\mathbb{L}$. Let $-R$ denote the reverse of $R$, i.e. $-R=\{(x, y):(y, x) \in R\}$. For candidates $x$ and $y$ let $\tau_{x y}$ denote the permutation on $A$ defined $\tau_{x y}(z)=z$ if $z \notin\{x, y\}, \tau_{x y}(x)=y$ and $\tau_{x y}(y)=x$. Now $\tau_{x y} R$ denotes the preference relation where the positions of $x$ and $y$ are swapped, i.e. $\tau_{x y} R=\left\{\left(\tau_{x y}(v), \tau_{x y}(w)\right):(v, w) \in R\right\}$.

A profile $p$ (of individual preferences) assigns to every voter $i$ a linear order $p(i)$ in $\mathbb{L}$. Let $\mathbb{L}^{N}$ denote the set of all these profiles, that is the set of all the combinations of individual

\footnotetext{
${ }^{2}$ If $x=y$, then $x$ and $y$ are indifferent. If $x \neq y$, then $x$ is strictly preferred to $y$.
} 
preferences. To exclude discussions on resolving ties in the collective orders we allow the collective outcomes to be weak orders, i.e. complete and transitive orders on $A$. The set of all these weak orders is denoted by $\mathbb{W}$. Further, $\tau_{x y} p$ is the coordinate-wise extension of $\tau_{x y}$. So, $\left(\tau_{x y} p\right)(i)=\tau_{x y}(p(i))$. For non-empty subsets of voters $S$ profiles $q$ and $p$ are called $S$-deviations if $p(i)=q(i)$ for all $i \in N-S$. Further, $\left.p\right|_{S}$ denotes the profile $p$ restricted to $S$.

For candidates $x$ and $y$ let $\mathbb{L}_{x y}$ denote the set of linear orders $R$ at which $x$ is strictly preferred to $y$, that is $\mathbb{L}_{x y}=\{R \in \mathbb{L} \mid(x, y) \in R\}$.

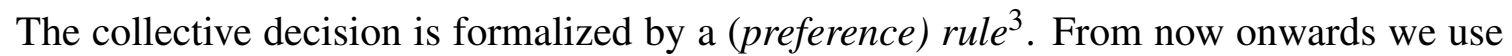
just rule instead of preference rule. $F$, a function that assigns to every profile $p$ in $\mathbb{L}^{N}$ a collective preference $F(p)$ in $\mathbb{W}$.

\section{Unimodal Frequency Distributions}

A frequency distribution is formalized by a function representing the number of times each linear order appear in a profile. Given a profile $p$ of individual preferences of the voters and a preference $R$ in $\mathbb{L}$, then $f(R, p)$ denotes the number of voters with preference $R$ at profile $p$, that is

$$
f(R, p)=|\{i \in N \mid p(i)=R\}|,
$$

where $|S|$ denotes the cardinality of an arbitrary set $S$. There is a metric space over $\mathbb{L}$ induced by the Kemeny distance function $d$ defined for two preferences $R^{1}$ and $R^{2}$ in $\mathbb{L}$ as follows ${ }^{\text {प1 }}$

$$
d\left(R^{1}, R^{2}\right)=\frac{1}{2}\left|\left(R^{1} \triangle R^{2}\right)\right|
$$

where $\triangle$ denotes the symmetric difference between sets, i.e. $R^{1} \triangle R^{2}=\left(R^{1}-R^{2}\right) \cup\left(R^{2}-\right.$ $R^{1}$ ). The Kemeny distance indicates the number of discordant pairs.

\footnotetext{
${ }^{3}$ Preference rules are usually known as Social Welfare Functions. As the model discussed here also applies to non-welfare issues such as voting we rather use the term preference rule.

${ }^{4}$ Here we actually take half the Kemeny distance because between two linear orderings it is a multiple of two.
} 
A profile $p$ is called unimodal if there exists a preference $\widehat{R}$, the mode, such that for every two preferences $R^{1}$ and $R^{2}$ in $\mathbb{L}$, such that $d\left(\widehat{R}, R^{1}\right)<d\left(\widehat{R}, R^{2}\right)$, then we have $f\left(R^{1}, p\right)>$ $f\left(R^{2}, p\right)$.

So, a profile $p$ is unimodal if there is a preference $\widehat{R}$ in $\mathbb{L}$ with highest frequency, such that frequencies for all other preferences in $\mathbb{L}$ strictly decrease in their distance from this mode $\widehat{R}$. A unimodal distribution is called symmetrical if in addition $f\left(R^{1}, p\right)=f\left(R^{2}, p\right)$ whenever $d\left(\widehat{R}, R^{1}\right)=d\left(\widehat{R}, R^{2}\right)$.

Example 4.1. Example of a symmetric unimodal distribution with 4 candidates $a, b, c, d$.

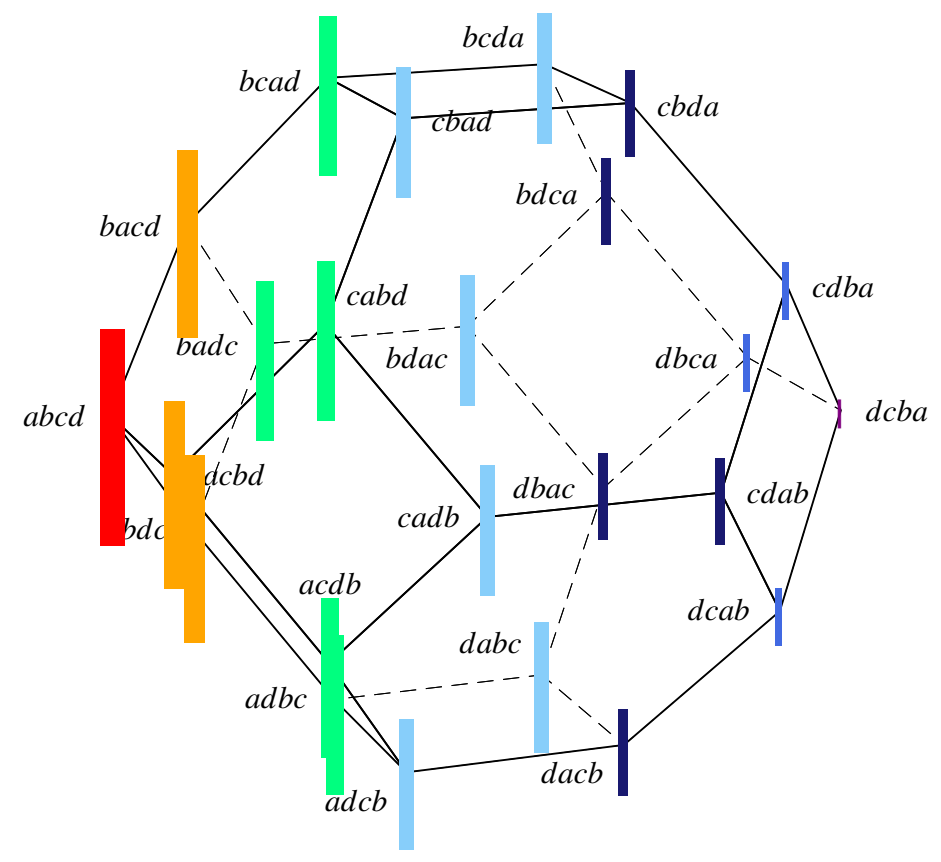

Figure 4.1: Symmetric unimodal distribution with 4 candidates

For 4 candidates we have 4 ! = 24 many linear orders in the set $\mathbb{L}$. This set $\mathbb{L}$ can be structured by the Kemeny distance as is shown in the above figure 4.1: the truncated octahedron. In this structure, an edge between two linear orders means that these have Kemeny distance 1, i.e. they differ by only one swap of consecutively ordered candidates. For instance, take $a b c d$ and bacd. Now we have a frequency distribution on this structure with mode at $a b c d$. The frequency of abcd is indicated by the length of the red bar. Going one step (in the Kemeny sense) away from $a b c d$, we have three linear orders, namely, 
$b a c d, a c b d, a b d c$. Their frequencies are lesser compared the mode. For this example, we have taken equal frequencies, so the distribution is symmetric. Their frequencies are indicated by the length of the yellow bars. Similarly we have declining frequencies for the linear orders at distances 2,3,4,5 and 6 from the mode $a b c d$.

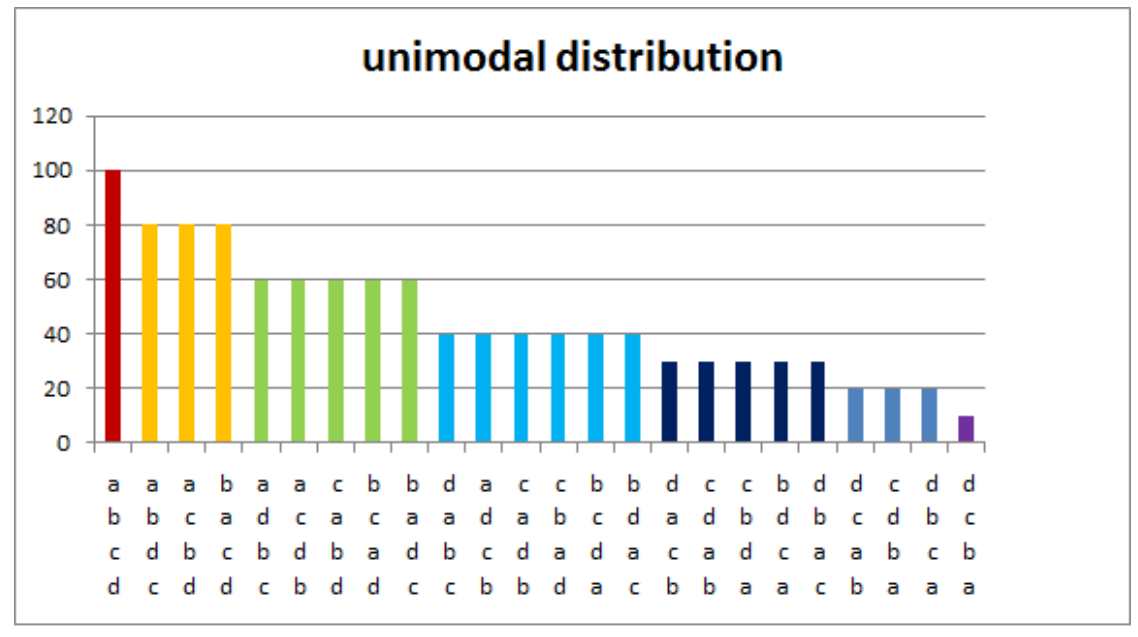

Figure 4.2: Symmetric unimodal distribution with 4 candidates

This same distribution can be viewed as a flat bar chart, where we use the same colors to indicate that those linear orders are at the same distance from the mode. Of course a lot of information is lot transferring from the Kemeny structure to 2 dimensional bar chart.

\section{Definitions of Some Conditions}

Preference rules will be discussed with respect to the following conditions.

Anonymity means that the rule is symmetric in its arguments. Anonymity guarantees that every voters' preference is equally important in determining the outcome. The outcome is based on how many times a preference is announced at a certain profile instead of who announced that preference. Let $\sigma: N \rightarrow N$ be a permutation on $N$ the set of voters. Let $p$ be a profile in $\mathbb{L}^{N}$. Then profile $\mathrm{e}^{\mathrm{\Xi}} q=p \circ \sigma$ is defined for all voters $i$ by

$$
q(i)=p(\sigma(i))
$$

\footnotetext{
${ }^{5}$ Note that we consider a profile to be a function from $N$ to $\mathbb{L}$.
} 
Rule $F$ is anonymous if for all profiles $p$ and all permutations $\sigma$

$$
F(p)=F(p \circ \sigma)
$$

Neutrality means that the rule treats candidates in equal situations equally. Let $x$ and $y$ be candidates.

Let $\tau_{x y}$ be a permutation on $A$, where it swaps the positions of $x, y \in A$. Rule $F$ is neutral if for all candidates $x$ and $y$, all profiles $p$ and all $\tau_{x y}$ on $A$

$$
F\left(\tau_{x y} p\right)=\tau_{x y} F(p)
$$

Monotonicity means that if in comparison of profiles $p$ and $q$ the preference " $x$ is preferred to $y$ " among the voters increases when going from $p$ to $q$, then this preference at the outcomes should not decrease.

Rule $F$ is monotone if for all candidates $x$ and $y$, all non-empty subsets $S$ of $N$ and all $S$ deviations $p$ and $q$, such that both $q(i)=\tau_{x y} p(i)$ and $(y, x) \in p(i)$ for all $i \in S(y, x) \notin F(p)$ implies $(y, x) \notin F(q)$.

Discrimination means that for candidates $x$ and $y$ at a profile $p$ the outcome $F(p)$ is indifferent between these two only if there are $R^{1}$ at which $x$ is strictly preferred to $y$ and $R^{2}$ at which $y$ is strictly preferred to $x$ such that $f\left(R^{t}, p\right) \geqslant f\left(\tau_{x y} R^{t}, p\right)$ for both $t \in\{1,2\}$. So, indifference between $x$ and $y$ in an outcome can only occur if neither for all $R \in \mathbb{L}_{x y}$ frequencies $f(R, p)$ are strictly greater than $f\left(\tau_{x y} R, p\right)$ nor for all $R \in \mathbb{L}_{y x}$ frequencies $f(R, p)$ are strictly greater than $f\left(\tau_{x y} R, p\right)$.

Rule $F$ discriminates if for all profiles $p$ and all different candidates $x$ and $y$

$(x, y) \in F(p)$ and $(y, x) \in F(p)$ implies , that there are preferences $R^{1} \in \mathbb{L}_{x y}$ and $R^{2} \in \mathbb{L}_{y x}$ with $f\left(R^{t}, p\right) \geqslant f\left(\tau_{x y} R^{t}, p\right)$ for all both $t \in\{1,2\}$.

It should be clear, that one equality between frequencies $f(R, p)$ and $f\left(\tau_{x y} R, p\right)$ for some linear order is sufficient to allow for an indifference outcome between $x$ and $y$ at a discriminating rule. This is also allowed in cases where $f\left(R^{1}, p\right)>f\left(\tau_{x y} R^{1}, p\right)$ and $f\left(R^{2}, p\right)>f\left(\tau_{x y} R^{2}, p\right)$ for some $R^{1} \in \mathbb{L}_{x y}$ and some $R^{2} \in \mathbb{L}_{y x}$. But in cases where

\footnotetext{
${ }^{6}$ The neutrality notion defined here is equivalent to the usual one, because every permutation can be decomposed into pairwise swaps like $\tau_{x y}$.
} 
$f(R, p)>f\left(\tau_{x y} R, p\right)$ for all $R \in \mathbb{L}_{x y}$ discrimination implies the preference between $x$ and $y$ is strict. It is natural to go one step further in those cases and to impose, that in those situations $x$ should be strictly preferred to $y$. This condition is referred to as positive discrimination.

So, rule $F$ positively discriminates if for all profiles $p$ and all different candidates $x$ and $y$ $(x, y) \in F(p)$ and $(y, x) \notin F(p)$ whenever $f(R, p)>f\left(\tau_{x y} R, p\right)$ for all $R \in \mathbb{L}_{x y}$.

As we frequently use the terms Condorcet consistent rules and pairwise rules in the following sections, let us clarify what we mean by them. Firstly a pairwise comparison matrix is a square matrix with its $(i, j)^{t h}$ element as the number of voters preferring the $i^{t h}$ candidate to the $j^{\text {th }}$ candidate, and all the diagonal elements are zero. Pairwise rules are the rules that provide the same outcome for two different preference profiles as long as the pairwise comparison matrix resulting from these two preference profiles are the same. A Condorcet consistent choice rule chooses the Condorcet winner if it exists. In case of a Condorcet consistent preference rule, the Condorcet order is chosen if it exists. A pairwise rule may not be Condorcet consistent, for example, Borda rule is pairwise but it is not Condorcet consistent. It is not difficult to see, that score rules as well as Condorcet consistent rules or pairwise rules are positively discriminating and monotone. The proofs for this are essentially given in Examples 4.2 and 4.3 in Section 4.3 .

\section{Decisions in Unimodal Frequency Distributions}

The following examples show that, at unimodal distributions the mode is the outcome of several well-known decision rules like score rules, Condorcet consistent or pairwise rules and the Coombs rule. There are many more rules that have the mode as outcome at these frequency distributions. Instead of proving this we formulate sufficient conditions for an arbitrary rule to assign the mode at such frequency distributions. In all these examples let $\widehat{p}$ be a unimodal profile with mode $\widehat{R}$. The result of these examples are based on the following Lemma.

Lemma 4.1. Let $x$ and $y$ be two different preferences and $R^{1}, R^{2}$ be two linear orders, such that $(x, y) \in R^{1}$ and $(y, x) \in R^{2}$. Then $d\left(R^{1}, \tau_{x y} R^{2}\right)<d\left(R^{1}, R^{2}\right)$.

Proof. We partition $A \backslash\{x, y\}$ as follows: 


$$
\begin{aligned}
B_{1} & =\left\{a \in A:(a, x) \in R^{1} \text { and }(a, y) \in R^{2}\right\}, \\
B_{2} & =\left\{a \in A:(a, x) \in R^{1} \text { and }(a, x),(y, a) \in R^{2}\right\}, \\
B_{3} & =\left\{a \in A:(a, x) \in R^{1} \text { and }(x, a) \in R^{2}\right\}, \\
M_{1} & =\left\{a \in A:(x, a),(a, y) \in R^{1} \text { and }(a, y) \in R^{2}\right\}, \\
M_{2} & =\left\{a \in A:(x, a),(a, y) \in R^{1} \text { and }(a, x),(y, a) \in R^{2}\right\}, \\
M_{3} & =\left\{a \in A:(x, a),(a, y) \in R^{1} \text { and }(x, a) \in R^{2}\right\}, \\
W_{1} & =\left\{a \in A:(y, a) \in R^{1} \text { and }(a, y) \in R^{2}\right\}, \\
W_{2} & =\left\{a \in A:(y, a) \in R^{1} \text { and }(a, x),(y, a) \in R^{2}\right\} \text { and } \\
W_{3} & =\left\{a \in A:(y, a) \in R^{1} \text { and }(x, a) \in R^{2}\right\} .
\end{aligned}
$$

The following pictures the preferences $R^{1}, R^{2}$ and $\tau_{x y} R^{2}$.

$$
\begin{array}{ccccccc}
B_{1} \cup B_{2} \cup B_{3} & x & M_{1} \cup M_{2} \cup M_{3} & y & W_{1} \cup W_{2} \cup W_{3} & : & R^{1} \\
B_{1} \cup M_{1} \cup W_{1} & y & B_{2} \cup M_{2} \cup W_{2} & x & B_{3} \cup M_{3} \cup W_{3} & : & R^{2} \\
B_{1} \cup M_{1} \cup W_{1} & x & B_{2} \cup M_{2} \cup W_{2} & y & B_{3} \cup M_{3} \cup W_{3} & : & \tau_{x y} R^{2}
\end{array}
$$

Now

$$
\begin{aligned}
d\left(R^{1}, R^{2}\right)= & \frac{1}{2}\left|\left[\left(R^{1} \triangle R^{2}\right) \cap(A-\{x, y\})^{2}\right]\right|+\left|B_{2}\right|+2 \cdot\left|B_{3}\right|+ \\
& \left|M_{1}\right|+2 \cdot\left|M_{2}\right|+\left|M_{3}\right|+2 \cdot\left|W_{1}\right|+\left|W_{2}\right|+1 \\
= & d\left(R^{1}, \tau_{x y} R^{2}\right)+2 \cdot\left|M_{2}\right|+1 \\
& >d\left(R^{1}, \tau_{x y} R^{2}\right),
\end{aligned}
$$

which completes the proof.

Lemma 4.2. Let $x, y \in A$, with $(y, x) \notin \widehat{R}$ and let $R \in \mathbb{L}_{x y}$. Then $f(R, \widehat{p})>f\left(\tau_{x y} R, \widehat{p}\right)$.

Proof. By Lemma 4. D it follows that $d(\widehat{R}, R)<d\left(\widehat{R}, \tau_{x y} R\right)$ which easily yields the desired inequality by the unimodality of $\widehat{p}$.

Example 4.2. Condorcet consistent rules 
Condorcet consistent rules depend on pairwise majority comparisons of the candidates. In general these comparisons may yield cycles and rules therefore may differ in these situations: different Condorcet consistent rules break up cycles in different ways. If, however, at a certain profile pairwise majority comparisons yield a complete, strict and transitive order from overall winner (the Condorcet winner) to overall loser (the Condorcet loser), then this is the outcome of all these rules at that profile. We argue that at profile $\widehat{p}$ pairwise majority comparisons yield the mode $\widehat{R}$ as the Condorcet order. Herewith we show that all Condorcet consistent preference rules assign the mode at a unimodal profile. Let $(x, y) \in$ $\widehat{R}$, with $x \neq y$. It is sufficient to show that

$$
|\{i \in N:(x, y) \in \widehat{p}(i)\}|>|\{i \in N:(y, x) \in \widehat{p}(i)\}|
$$

Lemma 4.2 yields that $f(R, \widehat{p})>f\left(\tau_{x y} R, \widehat{p}\right)$ for all $R \in L_{x y}$. Since, $|\{i \in N:(x, y) \in \widehat{p}(i)\}|=$ $\sum_{R \in \mathbb{L}_{x y}} f(R, \widehat{p})$ and $|\{i \in N:(y, x) \in \widehat{p}(i)\}|=\sum_{R \in \mathbb{L}_{x y}} f\left(\tau_{x y} R, \widehat{p}\right)$ the desired inequality follows.

\section{Example 4.3. Score Rules}

In score rules voters assign scores $s_{1}, s_{2}, \ldots, s_{m}$ to the candidates and these are then ordered according to their total scores. It is assumed that $s_{m} \geqslant s_{m-1} \geqslant \ldots \geqslant s_{1}$ and that $s_{m}>s_{1}$. At a preference $p(i)$ voter $i$ would hand out score $s_{m}$ to his best candidate, $s_{m-1}$ to his second best and so on. So, his worst candidate receives score $s_{1}$. Let $\operatorname{rank}(x, p(i))=\mid\{y \in A$ : $(x, y) \in p(i)\} \mid$ be the rank of candidate $x$ at preference $p(i)$. Then of course the rank of the best candidate is equal to $m$ the number of candidates and that of the worst candidate is equal to one. Voter $i$ assigns the scores as follows $s(x, p(i))=s_{\operatorname{rank}(x, p(i))}$ and total score for candidate $x$ at profile $p$ is now $s(x, p)=\sum_{i \in N} s(x, p(i))$. Define score rule $F_{s}$, with scores $s$, for candidates $x$ and $y$ and an arbitrary profile $p$ as follows

$$
(x, y) \in F_{s}(p) \text { if and only if } s(x, p) \geqslant s(y, p)
$$

In order to show that $F_{s}(\widehat{p})=\widehat{R}$, let $(x, y) \in \widehat{R}$ with $x \neq y$. It is sufficient to show that $s(x, \widehat{p})>s(y, \widehat{p})$. For numbers $v$ and $w$ let $\mathbb{L}_{x y}^{v w}=\left\{R \in \mathbb{L}_{x y}: \operatorname{rank}(x, R)=v\right.$ and $\operatorname{rank}(y, R)=$ $w\}$. Note that 


$$
\begin{aligned}
s(x, \widehat{p}) & =\sum_{v=2}^{v=m} \sum_{w=1}^{w=v-1}\left(\sum_{R \in \mathbb{L}_{x y}^{v w}} s_{v} \cdot f(R, \widehat{p})+\sum_{R \in \mathbb{L}_{y x}^{\nu w}} s_{w} \cdot f(R, \widehat{p})\right) \\
& =\sum_{v=2}^{v=m} \sum_{w=1}^{w=v-1}\left(\sum_{R \in \mathbb{L}_{x y}^{v w}} s_{v} \cdot f(R, \widehat{p})+s_{w} \cdot f\left(\tau_{x y} R, \widehat{p}\right)\right)
\end{aligned}
$$

and similarly

$$
s(y, \widehat{p})=\sum_{v=2}^{v=m} \sum_{w=1}^{w=v-1}\left(\sum_{R \in \mathbb{L}_{x y}^{v w}} s_{v} \cdot f\left(\tau_{x y} R, \widehat{p}\right)+s_{w} \cdot f(R, \widehat{p})\right) .
$$

Therefore it is sufficient to show that for all numbers $v$ and $w$, with $v>w$, and all $R \in \mathbb{L}_{x y}^{v w}$

$$
s_{v} \cdot f(R, \widehat{p})+s_{w} \cdot f\left(\tau_{x y} R, \widehat{p}\right) \geqslant s_{v} \cdot f\left(\tau_{x y} R, \widehat{p}\right)+s_{w} \cdot f(R, \widehat{p})
$$

and that at least one of these inequalities is strict. The latter equality is equivalent to

$$
\left(s_{v}-s_{w}\right) \cdot\left(f(R, \widehat{p})-f\left(\tau_{x y} R, \widehat{p}\right)\right) \geqslant 0
$$

Because $v>w$ we have that $s_{v} \geqslant s_{w}$, where by definition $s_{m}>s_{1}$. Lemma 4.2 yields that $f(R, \widehat{p})-f\left(\tau_{x y} R, \widehat{p}\right)>0$. So, the weak inequality follows readily. Strictness follows because at some preference $R$ candidate $x$ is ordered best and candidate $y$ is ordered worst.

\section{Sufficient Conditions for Choosing the Mode}

Here we state a set of sufficient conditions for arbitrary rules such that these have the mode as the outcome at unimodal preference distributions. As many rules satisfy these conditions we consider them as weak. Monotonicity as well as (positive) discrimination is closely related to the result spelled out by Lemma 4.1. 
Theorem 4.1. Let $F$ be a rule from $\mathbb{L}^{N}$ to $\mathbb{W}$ that is positively discriminating. Then, for every unimodal profile $p$ with mode $R$ it holds that $F(p)=R$.

Proof. It is sufficient to prove that $(x, y) \in F(p)$ and $(y, x) \notin F(p)$. Let $R \in \mathbb{L}_{x y}$. As Lemma 4.] implies that $d\left(\widehat{R}, \tau_{x y} R\right)>d(\widehat{R}, R)$, because $p$ is unimodal it follows for all $R \in \mathbb{L}_{x y}$, that $f(R, p)>f\left(\tau_{x y} R, p\right)$. But then $(x, y) \in F(p)$ and $(y, x) \notin F(p)$ because $F$ is positively discriminating.

Lemma 4.3. Let $F$ be a rule from $\mathbb{L}^{N}$ to $\mathbb{W}$ which is neutral, anonymous, monotone and discriminating. Then $F$ is positively discriminating.

Proof. In order to prove the second case let $F$ be anonymous, neutral, monotone and discriminating. Let $p$ be a profile and $x$ and $y$ be different candidates such that for all $R \in \mathbb{L}_{x y}$ frequencies $f(R, p)$ are strictly greater than $f\left(\tau_{x y} R, p\right)$. It is sufficient to prove that $(x, y) \in F(p)$ and $(y, x) \notin F(p)$. By discrimination of $F$ we have that $(x, y) \notin F(p)$ or $(y, x) \notin F(p)$. To the contrary suppose $(x, y) \notin F(p)$. It is sufficient to prove a contradiction. As $F(p)$ is a weak order, hence complete, $(y, x) \in F(p)$ and $(x, y) \notin F(p)$. Furthermore, neutrality implies $(y, x) \notin F\left(\tau_{x y} p\right)$ and $(x, y) \in F\left(\tau_{x y} p\right)$. Now for all $R \in \mathbb{L}_{x y}$

$$
f\left(\tau_{x y} R, \tau_{x y} p\right)=f(R, p)>f\left(\tau_{x y} R, p\right)=f\left(R, \tau_{x y} p\right) .
$$

This means that at profile $\tau_{x y} p$ every $R \in \mathbb{L}_{x y}$ is outnumbered by the frequency of $\tau_{x y} R$ which is in $\mathbb{L}_{y x}$. Therefore at $\tau_{x y} p$ we can distinguish a non-empty subset $S$ of $N$ such that

1. $f\left(R,\left.\left(\tau_{x y} p\right)\right|_{N-S}\right)=f\left(\tau_{x y} R,\left.\left(\tau_{x y} p\right)\right|_{N-S}\right)$ for all $R \in \mathbb{L}_{x y}$ and

2. $(x, y) \notin \tau_{x y} p(i)$ for all $i \in S$.

Consider profile $q$ such that $q(j)=\tau_{x y} p(j)$ for all $j \in N-S$ and $q(i)=\tau_{x y}\left(\tau_{x y} p(i)\right)=p(i)$ for all $i \in S$. Monotonicity and $(y, x) \notin F\left(\tau_{x y} p\right)$ imply $(y, x) \notin F(q)$. Hence, $(x, y) \in F(q)$ and $(y, x) \notin F(q)$. Next we show that $f(R, q)=f(R, p)$ for all $R$ in $\mathbb{L}$. For $R$ in $\mathbb{L}_{x y}$ this follows because $f(R, q)=f\left(R,\left.q\right|_{S}\right)+f\left(R,\left.q\right|_{N-S}\right)=f\left(R,\left.p\right|_{S}\right)+f\left(R,\left.\left(\tau_{x y} p\right)\right|_{N-S}\right)=$ $f\left(R,\left.p\right|_{S}\right)+f\left(\tau_{x y} R,\left.\left(\tau_{x y} p\right)\right|_{N-S}\right)=f\left(R,\left.p\right|_{S}\right)+f\left(R,\left.p\right|_{N-S}\right)=f(R, p)$. For $R$ in $\mathbb{L}_{y x}$ note that $f\left(R,\left.p\right|_{S}\right)=f\left(R,\left.q\right|_{S}\right)=0$ as $(y, x) \notin p(i)$ by the choice of $S$. So, the sequence of equations above alters to $f(R, q)=f\left(R,\left.q\right|_{S}\right)+f\left(R,\left.q\right|_{N-S}\right)=f\left(R,\left.\left(\tau_{x y} p\right)\right|_{N-S}\right)=$ 
$f\left(\tau_{x y} R,\left.\left(\tau_{x y} p\right)\right|_{N-S}\right)=f\left(R,\left.p\right|_{S}\right)+f\left(R,\left.p\right|_{N-S}\right)=f(R, p)$. But then by anonymity we have $F(q)=F(p)$, which cannot be because $(x, y) \in F(q)$ and $(x, y) \notin F(p)$.

Remark 4.1. In light of Lemma A.3, Theorem 4.1 holds true if $F$ is neutral, anonymous, monotone and discriminating.

The following example shows that the conditions in Lemma 4.3 are logically independent.

Example 4.4. Independence of anonymity, neutrality, monotonicity and discrimination

Let the dictatorial rule $F_{\text {dict }, i}$ with dictator $i$ be defined for a profile $p$ as $F_{\text {dict }, i}(p)=p(i)$. So, this rule assigns the preference of voter $i$ independent of the preferences of all other voters. Note that $F_{d i c t, i}$ is neutral, monotone and discriminating but of course not anonymous.

For a weak order $R$ on $A$ let the constant rule $F_{\text {const }, R}$ be defined for a profile $p$ as $F_{\text {const }, R}(p)=R$. So, this rule assigns relation $R$ independent of the preferences of the voters. Note that if $R \in \mathbb{L}$, then $F_{\text {const }, R}$ is anonymous, monotone and discriminating but not neutral, where if $R=A \times A$, then $F_{\text {const }, A \times A}$ is anonymous, neutral and monotone, but not discriminating.

Let the reverse transitive closure of pairwise majority rule $F_{\text {odd }}$ be defined for a profile $p$ and a pair of candidates $x$ and $y$ as follows, $(x, y) \in F_{\text {odd }}(p)$ if and only if there are $y=z_{0}, z_{1}, \ldots, z_{k}=x$ such that for all $0<j \leqslant k$

$$
\left|\left\{i \in N:\left(z_{j}, z_{j-1}\right) \in p(i)\right\}\right| \geqslant\left|\left\{i \in N:\left(z_{j-1}, z_{j}\right) \in p(i)\right\}\right| .
$$

It is straightforward to prove that $F_{o d d}$ is neutral, anonymous and discriminating. It is clearly not monotone.

It is clear that for instance Condorcet consistent or pairwise rules as well as score rules are positively discriminating and therefore by Theorem 4.1 their outcome equals the mode at unimodal distributed profiles.

An application of the second case of Theorem 4.1 can be found in the following example.

Example 4.5. Score-wise Elimination

Consider score rules introduced in Example 4.3. Let $s^{k}=\left(s_{1}^{k}, s_{2}^{k}, \ldots, s_{k}^{k}\right) \in \mathbb{R}^{k}$ for each $k$ from 1 to $m$, with $s_{k}^{k} \geqslant s_{k-1}^{k} \geqslant \ldots \geqslant s_{1}^{k}$ and $s_{k}^{k}>s_{1}^{k}$. Based on these score vectors the rule 
sequentially eliminates those alternatives which have lowest score. Here for a subset $B$ of $A$ the scores are determined for an arbitrary candidate $b$ in $B$ and profile $p$ as follows

$$
\operatorname{score}\left(s^{k}, b,\left.p\right|_{B}\right)=\sum_{i \in N} \operatorname{score}\left(s^{k}, b,\left.p\right|_{B}\right),
$$

where $|B|=k, \operatorname{score}\left(s^{k}, b,\left.p\right|_{B}\right)=s_{r\left(x,\left.p(i)\right|_{B}\right)}^{k}$ and $r\left(x,\left.p(i)\right|_{B}\right)=\left|\left\{x \in B:\left.(b, x) \in p(i)\right|_{B}\right\}\right|$. So, in round 0 set $A_{0}=A$ and

$$
C_{0}(p)=\left\{a \in A: \operatorname{score}\left(s^{m}, x, p\right) \geqslant \operatorname{score}\left(s^{m}, a, p\right) \text { for all } x \in A\right\}
$$

Now for each proceeding round $l>0$ define $A_{l}=A_{l-1}-C_{l-1}(p)$, and take

$$
\begin{aligned}
& C_{l}(p)=\left\{a \in A_{l}: \operatorname{score}\left(s^{k}, x,\left.p\right|_{A_{l}}\right) \geqslant \operatorname{score}\left(s^{k}, a,\left.p\right|_{A_{l}}\right) \text { for all } x \in A_{l}\right\} \\
& \quad \text { and } A_{l+1}=A_{l}-C_{l}(p) \text { if }\left|A_{l}\right|=k>0 \\
& C_{l}(p)=A_{l+1}=\emptyset \text { else. }
\end{aligned}
$$

The outcome of the score-wise elimination rule $F_{\text {elimination }}$ is now defined for an arbitrary pair of candidates and profile $p$ as follows

$$
(x, y) \in F_{\text {elimination }}(p) \text { if and only if } y \in A_{l} \text { implies } x \in A_{l} \text { for all } l \geq 0 .
$$

In case $s^{k}$ equals the anti-plurality scores, i.e. $(1,1,1, \ldots, 1,1,0)$ for all $k$, this score-wise elimination equals Coombs rule, in case it equals Borda score, i.e. $(k, k-1, \ldots, 2,1)$ it equals Nanson rule. The class is much richer though as for instance resemblance between the score vectors at different rounds of $k$ need not exist. We end this example by arguing that all score-wise elimination rules assign the mode to unimodal profiles. The argument is based on Theorem 4.1 case 2. By definition these elimination rules are neutral and anonymous. Note that these rules are monotone. Taking profiles $p$ and $q$ and candidates $x$ and $y$ as in the definition. At every subset of candidates in which these two are present the scores of all others is not affected and that of $x$ is weakly increased where that of $y$ is weakly decreased while going from $p$ to $q$. Next we show that these rules are discriminating. Let at profile $p$ and at subset $B$, with $|B|=k$, candidates $x$ and $y$ both be eliminated at round $t$, where $p, x$ and $y$ are chosen as in the definition of discrimination. 
Then scores of these at $\left.p\right|_{B}$ are equal. Now as $s_{k}^{t}>s_{1}^{t}$ it cannot be that $f(R, p)>f\left(\tau_{x y} R, p\right)$ for all linear orders $R \in \mathbb{L}_{x y}$. As this would yield $f\left(\left.R\right|_{B},\left.p\right|_{B}\right)>f\left(\left.\left(\tau_{x y} R\right)\right|_{B}, p_{\left.\right|_{B}}\right)$ for all linear orders $R \in \mathbb{L}_{x y}$, which can both not hold because of score being equal at $\left.p\right|_{B}$ where $s_{t}^{t}>s_{1}^{t}$. Similarly not $f(R, p)>f\left(\tau_{x y} R, p\right)$ for all linear orders $R \in \mathbb{L}_{y x}$. So, we have that these rules are discriminating. By Theorem 4.1 case 2 they assign the mode at unimodal distributions.

\section{Robustness of the Mode-selection Property}

So far we have studied behaviour of well-known rules under the assumption that preference profiles have a unimodal distribution. In this section we relax this assumption and try to analyse how sensitive the results of Examples 4.J and 4.2 are to perturbations of unimodal distributions. We confine our study here to specific cases of perturbations and specific rules. Of course we tried to deduce the similar results for more abstract rules like a subclass of score rules or abstract rules satisfying the sufficient conditions of Theorem 4.1 Case 2, but were not able to do so. As for rules we only consider Borda, plurality and Condorcet consistent rules. So, within the class of score rules we take two 'extreme' cases: all scores are different with equal margins (Borda) and except for the highest score all other scores are equal (Plurality). We further only consider perturbations of the following kind. There is a linear order, say $R^{*}$, and a real number $v$ such that

1. Frequencies are constant at any given distance from $R^{*}$. So, $f\left(R^{1}, p\right)=f\left(R^{2}, p\right)$ whenever $d\left(R^{1}, R^{*}\right)=d\left(R^{2}, R^{*}\right)$ for all linear orders $R^{1}$ and $R^{2}$.

2. For linear orders $R^{1}, R^{2}$ and $R^{3}$, with $d\left(R^{1}, R^{*}\right)<d\left(R^{2}, R^{*}\right) \leqslant v<d\left(R^{3}, R^{*}\right)$ we have $f\left(R^{1}, p\right)>f\left(R^{2}, p\right)>f\left(R^{3}, p\right)$.

Such distribution behave like a unimodal distribution with mode $R^{*}$ till distance $v$ from the mode and thereafter frequency is bounded by the frequency at distance $\lfloor v\rfloor^{\square}$ but does not have to decline like in unimodal distributions. We call such distributions $v$-tail-perturbed unimodal distributions around $R^{*}$.

Let $\rho$ denote the radius distance, i.e. $\rho=\frac{1}{2} \delta$, where $\delta=\left(\begin{array}{c}m \\ 2\end{array}\right)$ denotes the diameter distance of $\mathbb{L}$. In all results we will take $\rho \leqslant v$. So, seen from $R^{*}$ these perturbations only occur

\footnotetext{
${ }^{7}\lfloor v\rfloor$ denotes the largest integer less than or equal to $v$.
} 
in the second half of the set of linear orders. At such distributions frequency depends on the distance to $R^{*}$. Further, at distances larger than radius distance, say $\delta-k$, frequency is lower than at the 'opposite' distance $k$.

The analysis needs some combinatorial results which are discussed in the Appendix. Also some additional notations related to a (distance) $k$, candidates $x$ and $y$, linear orders $R$ and rank number $r$ are needed.

- $\mathbb{L}^{k}=\left\{R \in \mathbb{L}: d\left(R, R^{*}\right)=k\right\}$, i.e. the set of all linear orders on distance $k$ from $R^{*}$. This set is equal to $\left\{R \in \mathbb{L}: d\left(R,-R^{*}\right)=\delta-k\right\}$. Further, $l(k)=\left|\mathbb{L}^{k}\right|$ denotes the cardinality of $\mathbb{L}^{k}$.

- $\mathbb{L}_{x y}^{k}=\mathbb{L}_{x y} \cap \mathbb{L}^{k}$, i.e. the set of linear orders preferring $x$ to $y$ on distance $k$ from $R^{*}$ and $l(k, x y)=\left|\mathbb{L}_{x y}^{k}\right|$ denotes the cardinality of $\mathbb{L}_{x y}^{k}$.

- Also throughout this section we write $f(R)$ instead of $f(R, p)$ and also write $f(k)$ to indicate the frequency of linear orders on distance $k$ from $R^{*}$.

- $l(k, x, r)$, i.e. number of all linear orders on distance $k$ from $R^{*}$, where rank of $x$ is $r$.

- Let $\bar{l}, \bar{\delta}$ and $\bar{\rho}$ refer function $l$ in the case of $m-1$ candidates, the diameter and the radius in that case respectively.

Example 4.6. Example of a tail-perturbed unimodal distribution with 4 candidates $a, b, c, d$.

The following figure shows an example of a tail-perturbed unimodal distribution with 4 candidates. Suppose $a b c d$ is the mode of the distribution. Thereafter frequencies decline till distance 3 (i.e., $v=3$ ). After that frequencies fluctuate having an upper bound : the frequency at distance 3 . 


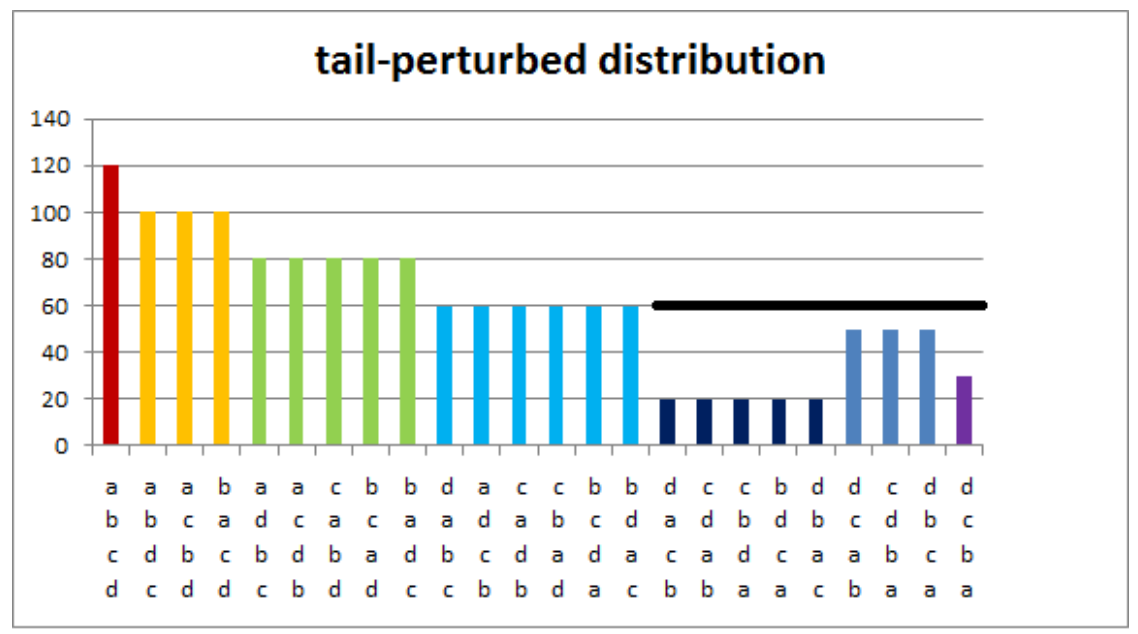

Figure 4.3: Tail-perturbed unimodal distribution

In the following theorem we show that at such perturbations Condorcet consistent rules choose $R^{*}$.

Theorem 4.2. Let $p$ have a $\rho$-tail-perturbed unimodal distributions around $R^{*}$. Then $R^{*}$ is the Condorcet order at $p$.

Proof. Let $(x, y) \in R^{*}$ for different candidates $x$ and $y$. It is sufficient to prove that $\sum_{R \in \mathbb{L}_{x y}} f(R)-f\left(\tau_{x y} R\right)>0$.

Note that

$$
\sum_{R \in \mathbb{L}_{x y}} f(R)-f\left(\tau_{x y} R\right)=\sum_{k=0}^{\delta}\left(\sum_{R \in \mathbb{L}_{x y}^{k}} f(R)-\sum_{R \in \mathbb{L}_{y x}^{k}} f(R)\right)
$$

As for linear order $R$ on distance $\rho$ from $R^{*}$ linear order $-R$ is also on distance $\rho$ from $R^{*}$ and frequencies are constant over a given distance we may exclude $k=\rho$ from this sum and split it up to yield 


$$
\begin{aligned}
\sum_{R \in \mathbb{L}_{x y}} f(R)-f\left(\tau_{x y} R\right)= & \sum_{0 \leq k<\rho}\left(\sum_{R \in \mathbb{L}_{x y}^{k}} f(R)-\sum_{R \in \mathbb{L}_{y x}^{k}} f(R)\right) \\
& +\sum_{0 \leq k<\rho}\left(\sum_{R \in \mathbb{L}_{x y}^{\delta-k}} f(R)-\sum_{R \in \mathbb{L}_{y x}^{\delta-k}} f(R)\right) \\
= & \sum_{0 \leq k<\rho} l(k, x y) f(k)-l(k, y x) f(k) \\
& +\sum_{0 \leq k<\rho} l(\delta-k, x y) f(\delta-k)-l(\delta-k, y x) f(\delta-k) .
\end{aligned}
$$

Where the latter equality follows by the assumptions on the frequency distribution. As further $l(k, x y)=l(\delta-k, y x)$ because $R \in \mathbb{L}_{x y}^{k}$ if and only if $-R \in \mathbb{L}_{y x}^{\delta-k}$ we have

$$
\begin{aligned}
\sum_{R \in \mathbb{L}_{x y}} f(R)-f\left(\tau_{x y} R\right) & =\sum_{0 \leq k<\rho} l(k, x y)[f(k)-f(\delta-k)]+l(k, y x)[(f(\delta-k)-f(k)] \\
& =\sum_{0 \leq k<\rho}[l(k, x y)-l(k, y x)][f(k)-f(\delta-k)] .
\end{aligned}
$$

Now by Lemma $4.9 l(k, x y) \geqslant l(k, y x)$ for all $0 \leq k \leq \rho$. The assumptions on the distribution show that $f(k)-f(\delta-k)$ is positive for all $0 \leq k \leq \rho$. For $k=0$ it is obvious that $l(k, x y)>l(k, y x)$ and $f(k)>f(\delta-k)$. So, $\sum_{R \in \mathbb{L}_{x y}} f(R)-f\left(\tau_{x y} R\right)$ is strictly positive.

Remark 4.2. In Theorem 4.2 the assumption on frequencies being constant on a given distance from $R^{*}$ can be relaxed and further looking at the proof frequencies at distance $\rho$ from $R^{*}$ need not be an upper bound for frequencies at greater distances. That is in the same way we can prove that $R^{*}$ is the Condorcet order if frequencies satisfy

$$
\min _{R \in \mathbb{L}_{x y}^{k}} f(R)+\min _{R \in \mathbb{L}_{x y}^{\delta-k}} f(R)>\max _{R \in \mathbb{L}_{y x}^{k}} f(R)+\max _{R \in \mathbb{L}_{y x}^{\delta-k}} f(R) \text { for all } 0 \leq k<\rho
$$

As by the second equation in the foregoing proof we have 


$$
\begin{aligned}
\sum_{R \in \mathbb{L}_{x y}} f(R)-f\left(\tau_{x y} R\right) & \geqslant \sum_{0 \leq k<\rho} l(k, x y) \cdot \min _{R \in \mathbb{L}_{x y}^{k}} f(R)-l(k, y x) \cdot \max _{R \in \mathbb{L}_{y x}^{k}} f(R) \\
& l(-k, x y) \cdot \min _{R \in \mathbb{L}_{x y}^{-k}} f(R)-l(-k, y x) \cdot \max _{R \in \mathbb{L}_{y x}^{-k}} f(R) \\
& =\sum_{0 \leq k<\rho} l(k, x y) \cdot\left(\min _{R \in \mathbb{L}_{x y}^{k}} f(R)-\max _{R \in \mathbb{L}_{y x}^{-k}} f(R)\right) \\
& -l(k, y x) \cdot\left(\max _{R \in \mathbb{L}_{y x}^{k}} f(R)-\min _{R \in \mathbb{L}_{x y}^{-k}} f(R)\right) .
\end{aligned}
$$

Now as $l(k, x y) \geqslant l(k, y x)$ and the assumption the result follows.

Example 4.7. Performance of Condorcet consistent rules under tail-perturbed unimodal distribution

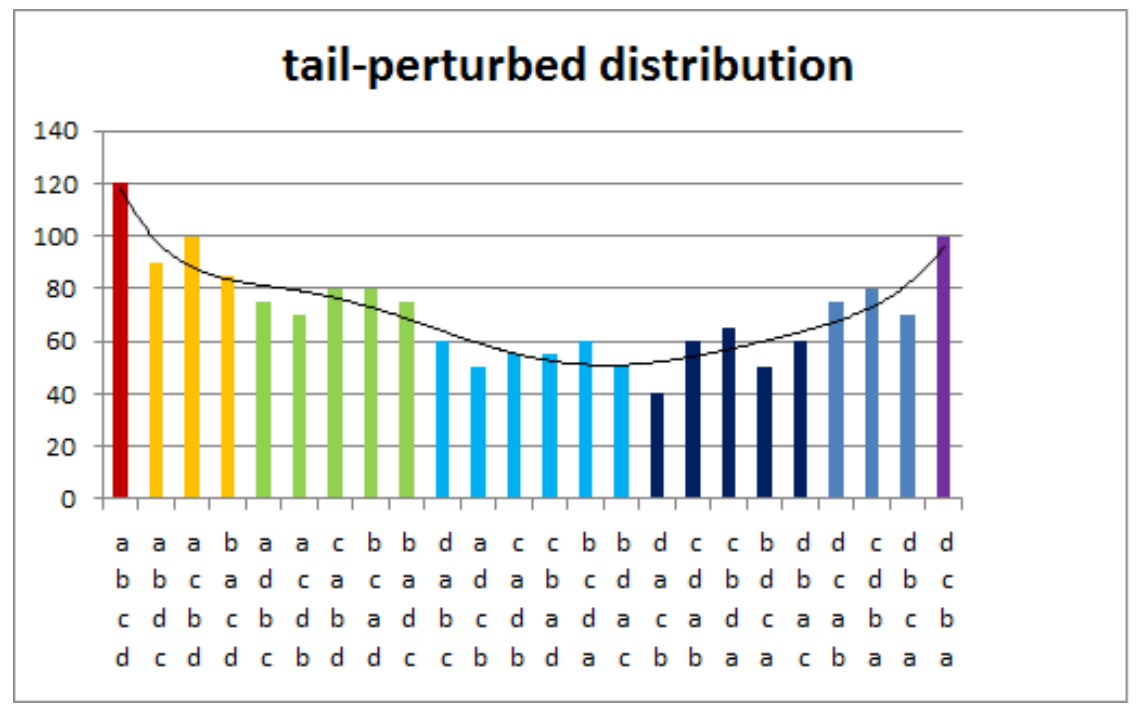

Figure 4.4: Robustness of Condorcet consistent rules

In the above figure 4.4, we show a voting situation with tail-perturbed unimodal frequencies satisfying the conditions given in remark 4.2. Here we consider a voting situation with 4 candidates $a, b, c, d$. abcd is the mode of the distribution. Thereafter frequencies decline till radius distance $\rho=3$. Here it is also possible to have different frequencies at 
the same distance from the mode. After radius frequencies are just bounded by the minimum frequency at the same distance from $d c b a$, the reverse mode. Also, till the radius, maximum frequency at any distance from the mode is lesser than the minimum frequency at any distances closer to the mode.

Next we check for sensitivity of the Borda rule. Borda rule is based on Borda score. Borda score of a candidate $x$ is the number of candidates below $x$ in a preference $R$, summed over all preferences. We show that for perturbed distributions discussed above, the Borda rule chooses $R^{*}$ as the outcome.

Theorem 4.3. Let $p$ have a $\rho$-tail-perturbed unimodal distributions around $R^{*}$. Then Borda rule assigns outcome $R^{*}$ to $p$.

Proof. Let scores $s_{t}$ be the Borda scores, i.e. $s_{t}=t$ for all $1 \leqslant t \leqslant m$. Take $(x, y) \in R^{*}$ arbitrary where $x$ and $y$ are different candidates. It is sufficient to prove that $s(x, p)>$ $s(y, p)$. Note for each distance $k$ to $R^{*}$ Borda score of candidate $x$ satisfies

$$
\sum_{z \in A} l(k, x z)=\sum_{r=1}^{m} r \cdot l(k, x, r) .
$$

So, Borda score of candidate $x$ equals

$$
\begin{aligned}
s(x, p) & =\sum_{k=0}^{\delta} \sum_{z \in A} f(k) \cdot l(k, x z) \\
& =\sum_{0 \leq k<\rho} \sum_{z \in A} f(k) \cdot l(k, x z)+f(\delta-k) \cdot l(\delta-k, x z) .
\end{aligned}
$$

Where the latter equation follows because $R$ is on distance $\rho$ from $R^{*}$ then so is $-R$. Noting that $l(\delta-k, b a)=l(k, a b)$ for all candidates $a$ and $b$ and all distances $k$ between 0 and $\rho$ we have 


$$
\begin{aligned}
s(x, p) & =\sum_{0 \leq k<\rho} \sum_{z \in A} f(k) \cdot l(k, x z)+f(\delta-k) \cdot l(\delta-k, x z) \\
& =\sum_{0 \leq k<\rho} \sum_{z \in A} f(k) \cdot l(k, x z)+f(\delta-k) \cdot l(\delta-k)-f(\delta-k) \cdot l(\delta-k, z x) \\
& =\sum_{0 \leq k<\rho} \sum_{z \in A}[f(k)-f(\delta-k)] \cdot l(k, x z)+f(\delta-k) \cdot l(\delta-k) .
\end{aligned}
$$

Hence,

$$
s(x, p)-s(y, p)=\sum_{0 \leq k<\rho} \sum_{z \in A}[f(k)-f(\delta-k)] \cdot[l(k, x z)-l(k, y z)]
$$

Note that for $k=0$ we obviously have that $\sum_{z \in A} l(k, x z)-l(k, y z)>0$. So, by the assumptions on frequency distribution $f$ and Lemma 4.10 we are done.

Similar to the case with Condorcet consistent rules, here also we mention a more general version of the result in the following Remark.

Remark 4.3. Looking at the proof of Theorem 4.3 it is clear that the same result can be deduced in case the distribution satisfies

$$
f(k)>f(\delta-k) \text { for all } 0 \leq k<\rho .
$$

Example 4.8. Performance of Borda rule under tail-perturbed unimodal distribution 


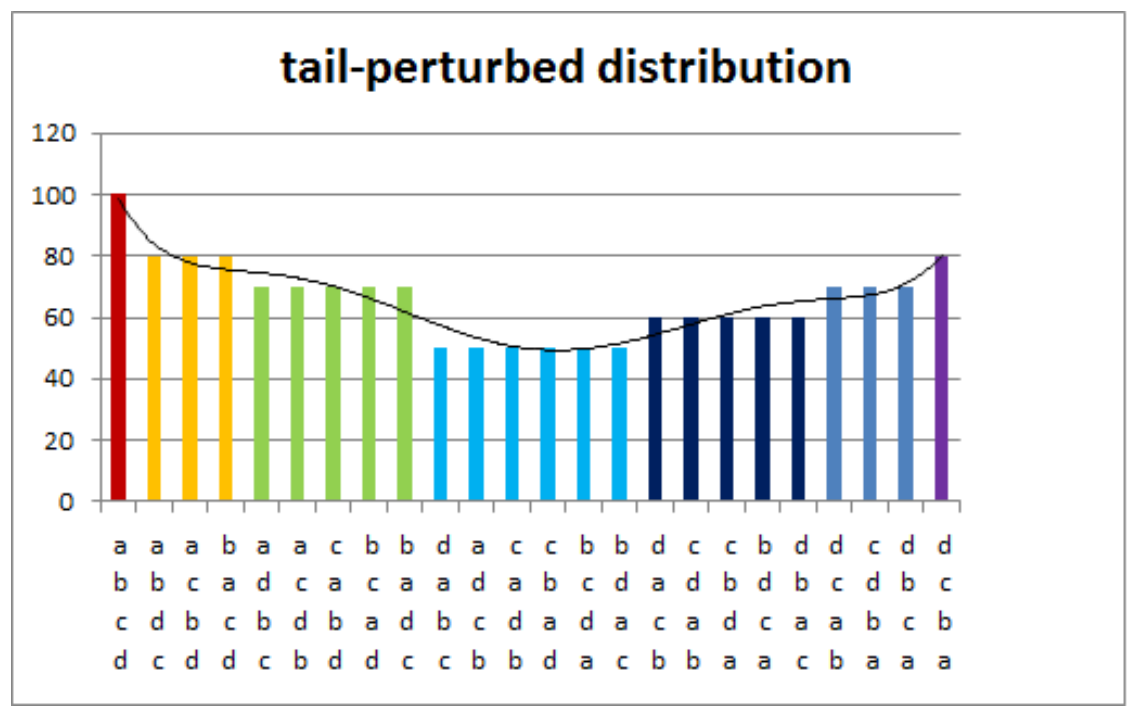

Figure 4.5: Robustness of Borda rule

In this example we show a voting situation with tail-perturbed unimodal frequencies satisfying the condition given in remark 4.3. Unlike example 4.7, here we have equal frequencies at the same distance from the mode.

Finally we show that the outcome of Plurality rule at $v$-tail-perturbed unimodal distributions towards $R^{*}$ is equal to $R^{*}$. We need $v$ to be equal to $\bar{\mu}=\frac{1}{2}\left(\begin{array}{c}m-1 \\ 2\end{array}\right)+m-\frac{3}{2}$.

Plurality rule is based on plurality score. That is the number of times a candidate is at the top of voter's preferences, i.e. having rank $m$ at the preference of an agent. It ranks the candidates according to their plurality scores. Consider profile $p$ having $\bar{\mu}$-tail-perturbed unimodal distributions towards $R^{*}$. Let $p l(x)$ denote this plurality score for candidate $x$. In this situation we have

$$
p l(x)=\sum_{k=0}^{\delta} l(k, x, m) \cdot f(k) .
$$

Let $r_{x}$ be the rank of $x$ in $R^{*}$. Then for any distance $k \geqslant u_{x}$, with $u_{x}=m-r_{x}$ we have that $l(k, x, m)=\bar{l}\left(k-u_{x}\right)$. Where $\bar{l}\left(k-u_{x}\right)$ denotes the number of preferences at distance $k-u_{x}$ from $\bar{R}^{*}$ the restriction of $R^{*}$ to $A-\{x\}$. This is because it needs precisely $u_{x}$ swaps to bring $x$ to the top at a preference when at start its rank is $r_{x}$. Denoting the diameter of the set of linear orders on $m-1$ candidates by $\bar{\delta}$ we therefore have 


$$
p l(x)=\sum_{k=u_{x}}^{\bar{\delta}+u_{x}} \bar{l}\left(k-u_{x}\right) \cdot f(k) .
$$

The above sum is over all $k \in\left\{u_{x}, u_{x}+1, \ldots, \bar{\delta}+u_{x}\right\}$, because if $k$ is outside this range, $\bar{l}\left(k-u_{x}\right)$ is not defined. Now we can prove the following result on Plurality rule.

Theorem 4.4. Let $p$ have a $\bar{\mu}$-tail-perturbed unimodal distributions around $R^{*}$. Then the outcome of Plurality rule at $p$ equals $R^{*}$.

Proof. Let $x$ and $y$ be different candidates such that $(x, y) \in R^{*}$. It is sufficient to prove $p l(x)-p l(y)>0$. Let $r_{x}$ and $r_{y}$ denote the rank of $x$ and $y$ in $R^{*}$ respectively. Let $u_{x}=$ $m-r_{x}$ and $u_{y}=m-r_{y} . r_{x}>r_{y}$ implies $u_{x}<u_{y}$. Then $p l(x)-p l(y)$ is equal to

$$
\sum_{k=u_{x}}^{\bar{\delta}+u_{x}} \bar{l}\left(k-u_{x}\right) \cdot f(k)-\sum_{k=u_{y}}^{\bar{\delta}+u_{y}} \bar{l}\left(k-u_{y}\right) \cdot f(k) .
$$

Take $\mu_{x y}$ the average of $\bar{\rho}+u_{x}$ and $\bar{\rho}+u_{y}$, so $\mu_{x y}=\bar{\rho}+\frac{1}{2}\left(u_{x}+u_{y}\right)$, where $\bar{\rho}$ is the radius distance of the set of linear orders on $m-1$ candidates. Defining $\bar{l}(k)$ to be zero if $k$ is not in the interval $[0, \bar{\delta}]$ we have that $p l(x)-p l(y)$ is equal to

$$
\sum_{u_{x} \leqslant k \leq \mu_{x y}}\left[\bar{l}\left(k-u_{x}\right)-\bar{l}\left(k-u_{y}\right)\right] \cdot f(k)+\sum_{\mu_{x y}<k \leq \bar{\delta}+u_{y}}\left[\bar{l}\left(k-u_{x}\right)-\bar{l}\left(k-u_{y}\right)\right] \cdot f(k) .
$$

For $k=\mu_{x y}=\bar{\rho}+\frac{1}{2}\left(u_{x}+u_{y}\right)$ we have $\bar{l}\left(k-u_{x}\right)=\bar{l}\left(\bar{\rho}+\frac{1}{2}\left(u_{y}-u_{x}\right)\right)$ and $\bar{l}\left(k-u_{y}\right)=\bar{l}(\bar{\rho}-$ $\left.\frac{1}{2}\left(u_{y}-u_{x}\right)\right)$. As $\bar{l}$ is symmetric around $\bar{\rho}$ it follows that $\left[\bar{l}\left(k-u_{x}\right)-\bar{l}\left(k-u_{y}\right)\right] \cdot f(k)$ is zero for $k=\mu_{x y}$. So, $p l(x)-p l(y)$ is equal to

$$
\sum_{u_{x} \leqslant k<\mu_{x y}}\left[\bar{l}\left(k-u_{x}\right)-\bar{l}\left(k-u_{y}\right)\right] \cdot f(k)+\sum_{\mu_{x y}<k \leq \bar{\delta}+u_{y}}\left[\bar{l}\left(k-u_{x}\right)-\bar{l}\left(k-u_{y}\right)\right] \cdot f(k)
$$


Substituting $k^{\prime}=\bar{\delta}+u_{x}+u_{y}-k$,

$$
\begin{aligned}
= & \sum_{u_{x} \leqslant k<\mu_{x y}}\left[\bar{l}\left(k-u_{x}\right)-\bar{l}\left(k-u_{y}\right)\right] \cdot f(k)+ \\
& \sum_{u_{x} \leqslant k^{\prime}<\mu_{x y}}\left[\bar{l}\left(\left(\overline{\boldsymbol{\delta}}+u_{y}\right)-\left(k^{\prime}-u_{x}\right)-u_{x}\right)-\bar{l}\left(\left(\overline{\boldsymbol{\delta}}+u_{y}\right)-\left(k^{\prime}-u_{x}\right)-u_{y}\right)\right] \cdot f\left(\left(\overline{\boldsymbol{\delta}}+u_{y}\right)-\left(k^{\prime}-u_{x}\right)\right) .
\end{aligned}
$$

As

$$
\begin{aligned}
& \bar{l}\left(\left(\overline{\boldsymbol{\delta}}+u_{y}\right)-\left(k^{\prime}-u_{x}\right)-u_{x}\right)-\bar{l}\left(\left(\overline{\boldsymbol{\delta}}+u_{y}\right)-\left(k^{\prime}-u_{x}\right)-u_{y}\right) \cdot f\left(\left(\overline{\boldsymbol{\delta}}+u_{y}\right)-\left(k^{\prime}-u_{x}\right)\right) \\
& =\left[\bar{l}\left(\overline{\boldsymbol{\delta}}+u_{y}-k^{\prime}\right)-\bar{l}\left(\overline{\boldsymbol{\delta}}+u_{x}-k^{\prime}\right)\right] \cdot f\left(\overline{\boldsymbol{\delta}}+u_{x}+u_{y}-k^{\prime}\right)
\end{aligned}
$$

and $\bar{l}\left(\bar{\delta}+u_{y}-k^{\prime}\right)=\bar{l}\left(k^{\prime}-u_{y}\right)$ and $\bar{l}\left(\bar{\delta}+u_{x}-k^{\prime}\right)=\bar{l}\left(k^{\prime}-u_{x}\right)$ it follows that

$$
\begin{aligned}
p l(x)-p l(y)= & \sum_{u_{x} \leqslant k<\mu_{x y}}\left[\bar{l}\left(k-u_{x}\right)-\bar{l}\left(k-u_{y}\right)\right] \cdot f(k)+ \\
& \sum_{u_{x} \leqslant k<\mu_{x y}}\left[\bar{l}\left(k-u_{y}\right)-\bar{l}\left(k-u_{x}\right)\right] \cdot f\left(\bar{\delta}+u_{x}+u_{y}-k\right) .
\end{aligned}
$$

So,

$$
p l(x)-p l(y)=\sum_{u_{x} \leqslant k<\mu_{x y}}\left[\bar{l}\left(k-u_{x}\right)-\bar{l}\left(k-u_{y}\right)\right] \cdot\left[f(k)-f\left(\bar{\delta}+u_{x}+u_{y}-k\right)\right] .
$$

By Lemma 4.11 it follows that the first factor in each term of this latter summation is positive and by the assumptions on $f$ that the second factor is positive. All in all therefore $p l(x)-p l(y)>0$ which completes the proof.

Example 4.9. Performance of Plurality rule under tail-perturbed unimodal distribution 


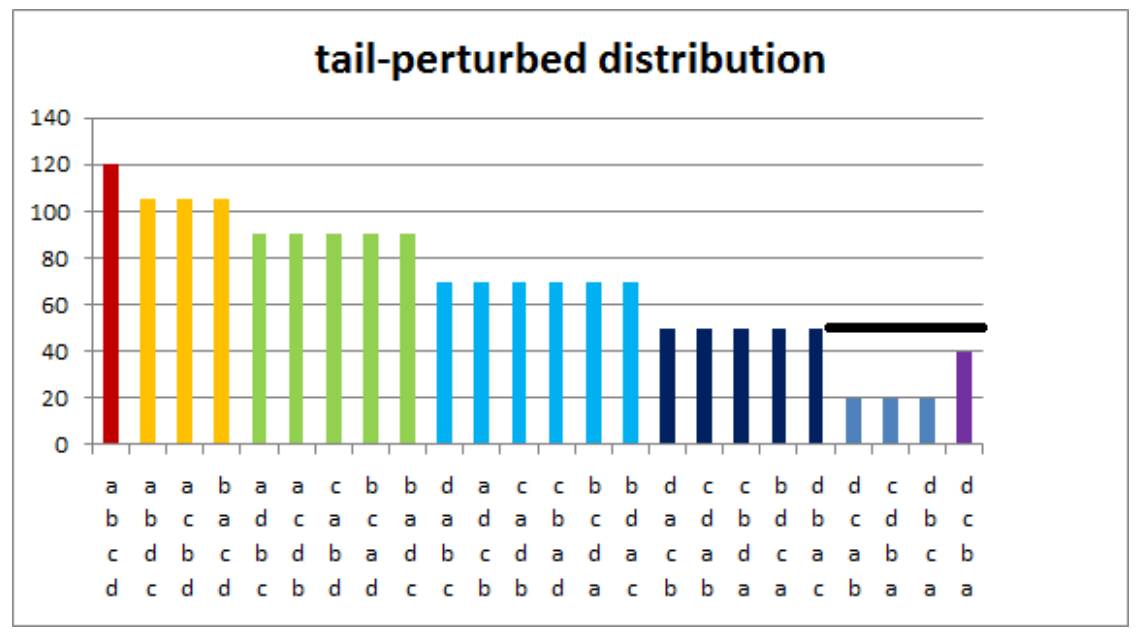

Figure 4.6: Robustness of Plurality rule

Here is a voting situation with tail-perturbed unimodal frequencies satisfying conditions given in theorem 4.4. For 4 candidates $a, b, c, d$ we have $\bar{\mu}=4$. So, disturbances happen only after distance 4 from the mode $a b c d$.

In the following examples we show that the conditions for choosing $R^{*}$ by Condorcet consistent, Borda and Plurality rules are not necessary. We consider a voting scenario with three alternatives $a, b$ and $c$. The preference distribution is given in the table below:

\begin{tabular}{|c|c|c|c|c|c|c|}
\hline Preferences & abc & acb & cab & cba & bca & bac \\
\hline Frequencies & $\mathrm{f}(0)$ & $\mathrm{f}(1)$ & $\mathrm{f}(2)$ & $\mathrm{f}(3)$ & $\mathrm{f}(2)$ & $\mathrm{f}(1)$ \\
\hline
\end{tabular}

Table 4.1: Frequency distribution with three alternatives

Thus we have the following table showing Pairwise comparisons. 


\begin{tabular}{|c|c|c|c|}
\hline Alt & Alt & a & c \\
\hline$a$ & - & $\mathrm{f}(0)+\mathrm{f}(1)+\mathrm{f}(2)$ & $\mathrm{f}(0)+2 \mathrm{f}(1)$ \\
\hline$b$ & $\mathrm{f}(1)+\mathrm{f}(2)+\mathrm{f}(3)$ & - & $\mathrm{f}(0)+\mathrm{f}(1)+\mathrm{f}(2)$ \\
\hline$c$ & $2 \mathrm{f}(2)+\mathrm{f}(3)$ & $\mathrm{f}(1)+\mathrm{f}(2)+\mathrm{f}(3)$ & - \\
\hline
\end{tabular}

Table 4.2: Pairwise comparison

Example 4.10. Suppose that the following conditions are satisfied

1. $f(0)>f(3)$,

2. $f(2)>f(1)$,

3. $f(0)-f(3)>2(f(2)-f(1))$.

Condition 3 does not comply with the conditions in Theorems 4.2,4.3. But, from Table 4.2 it is clear that Condorcet-like rules and Borda rule ranks the alternatives as $a \succ b \succ c$ as it was in the pivotal preference $a b c$.

Example 4.11. Suppose that the frequencies satisfy the following conditions:

1. $f(0)>f(2)$,

2. $f(1)>f(3)$,

3. $f(1)<f(2)$.

Condition 3 does not comply with the conditions in Theorem 4.4. But Plurality rule ranks the alternatives as $a \succ b \succ c$ as it was in the pivotal preference $a b c$.

\section{Multimodal Frequency Distributions}

Election outcomes often yield vote accumulations around several candidates or parties. It is therefore possible that these consist of several unimodal distributions superposed on 
each other, where their modes represent these spots of accumulation. In this section we study the case where two unimodal distributions are superposed on each. Here positively discriminating rules choose an outcome between these two modes. The notion of betweenness means that the Kemeny distances between the outcome of the rule to each mode precisely adds up to the distance between these two modi. So, the outcome can be seen as a convex combination (in the Kemeny sense) of the two modes.

Let $N_{1}$ and $N_{2}$ be two disjoint sets of voters. Let $p^{1} \in \mathbb{L}^{N_{1}}$ and $p^{2} \in \mathbb{L}^{N_{2}}$ such that $p^{t}$ is a unimodal profile on $N_{t}$ with mode $R^{t}$ for $t \in\{1,2\}$. Let $N=N_{1} \cup N_{2}$ and $p \in \mathbb{L}^{N}$ defined by $p(i)=p^{t}(i)$ if $i \in N_{t}$ for $t \in\{1,2\}$. In that case $p$ is said to be a superposition of unimodal profiles $p^{1}$ and $p^{2}$. It is straightforward to see that on such superposed profiles different rules may result in different outcomes. On the other hand the following theorem shows that positively discriminating preference rules agree on the intersection of these two modes.

Example 4.12. Example of multimodal distribution with 3 candidates $a, b, c$.

\begin{tabular}{|c|c|c|c|}
\hline Preferences & $\mathbf{p}$ & $\mathbf{q}$ & $\mathbf{p + q}$ \\
\hline$a b c$ & 100 & 80 & 180 \\
$a c b$ & 80 & 60 & 140 \\
$b a c$ & 80 & 100 & 180 \\
$c a b$ & 60 & 20 & 80 \\
$b c a$ & 60 & 80 & 140 \\
$c b a$ & 20 & 60 & 80 \\
\hline
\end{tabular}

Table 4.3: Multimodal distribution

This example is to show how to form a multimodal distribution from two unimodal ones. We have 3 candidates $a, b, c$. So, the first column in table 4.3 lists the 6 possible linear orders. The second column shows the frequencies corresponding to profile $p$. Notice that, $a b c$ is the mode at profile $p$. Similarly $b a c$ is the mode at profile $q$ shown in the third column. 

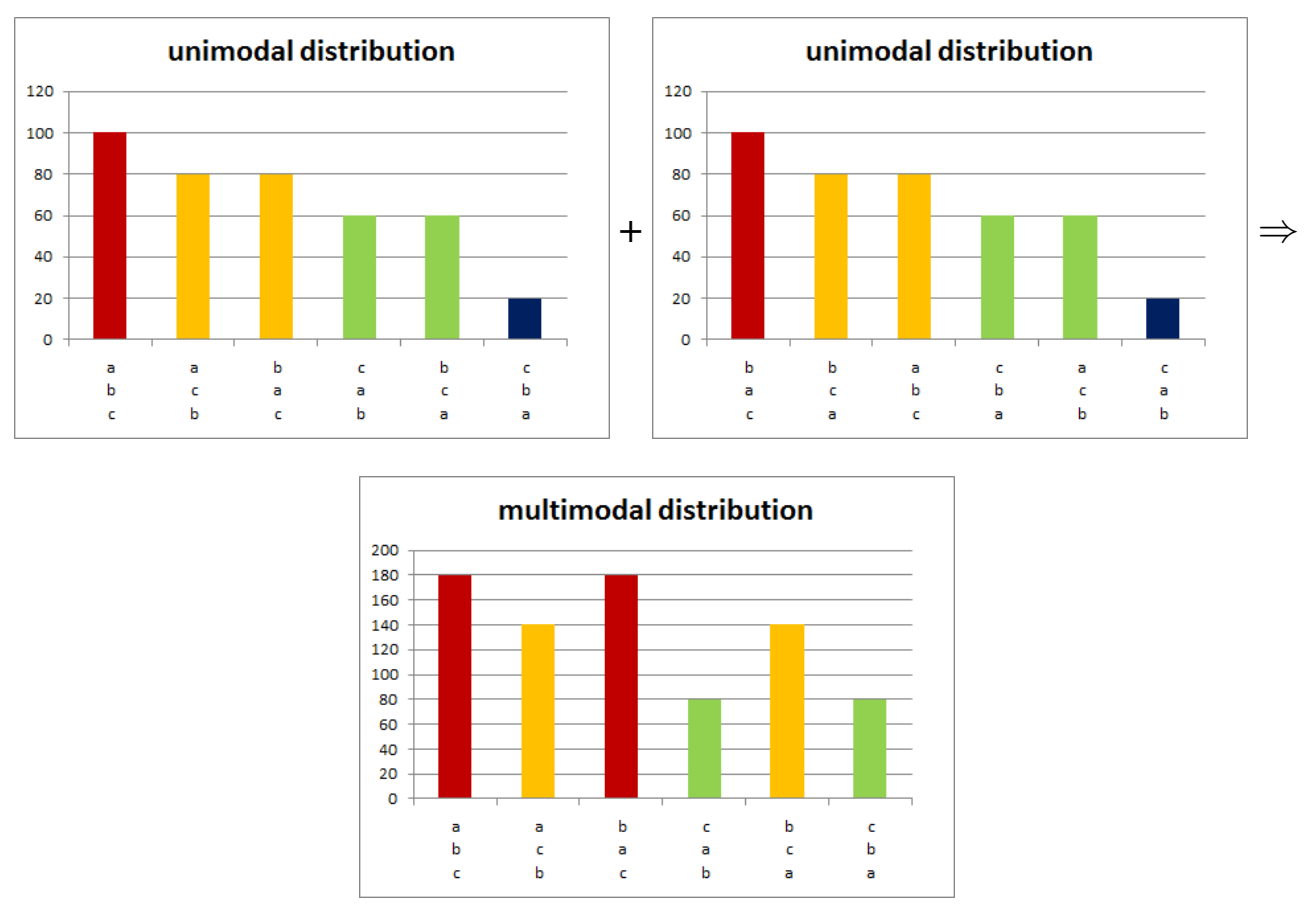

Figure 4.7: Multimodal distribution

Now, adding the corresponding frequencies from $p$ and $q$ we have a new profile $p+q$ shown in column 4. $p+q$ has two modes, namely $a b c$ and $b a c$. The same scenario is represented in the above figure 4.7 .

Theorem 4.5. Let $N_{1}$ and $N_{2}$ be two disjoint non-empty sets of voters, such that $N=$ $N_{1} \cup N_{2}$. For $t \in\{1,2\}$ and let $p^{t}$ be a unimodal profile on $N_{t}$ with mode $R^{t}$. Let $p$ be the superposition of these two profiles. Let $F$ be a positively discriminating rule from $\mathbb{L}^{N}$ to $\mathbb{W}$.

Then $(x, y) \in F(p)$ and $(y, x) \notin F(p)$ for all $(x, y) \in R^{1} \cap R^{2}$, such that $x \neq y$.

Proof. Let $(x, y) \in R^{1} \cap R^{2}$, with $x \neq y$. It is sufficient to prove that $(x, y) \in F(p)$ and $(y, x) \notin F(p)$. Because $R^{1}$ and $R^{2}$ are linear orders it follows that $(y, x) \notin R^{1}$ and $(y, x) \notin R^{2}$ . Lemma 4.2 implies for all $R \in \mathbb{L}_{x y}$ that

$$
f\left(R, p^{1}\right)>f\left(\tau_{x y} R, p^{1}\right) \text { and } f\left(R, p^{2}\right)>f\left(\tau_{x y} R, p^{2}\right)
$$


Therefore

$$
f(R, p)=f\left(R, p^{1}\right)+f\left(R, p^{2}\right)>f\left(\tau_{x y} R, p^{1}\right)+f\left(\tau_{x y} R, p^{2}\right)=f\left(\tau_{x y} R, p\right) .
$$

Positive discrimination implies that $(x, y) \in F(p)$ and $(y, x) \notin F(p)$.

Remark 4.4. Theorem 4.5] shows that in case of two superposed unimodal distributions, $p$, with modi $R^{1}$ and $R^{2}$ the asymmetric part of the outcome, $F(p)$, of a positively discriminating rule, $F$, contains the intersection of these two modi. Because the modi are complete and antisymmetric it follows that

$$
\left(F(p) \triangle R^{1}\right) \cup\left(F(p) \triangle R^{2}\right)=\left(R^{1} \triangle R^{2}\right) .
$$

So, $F(p)$ is on a shortest path between $R^{1}$ and $R^{2}$.

By Lemma 4.3 this result is also implied if the rule is simultaneously neutral anonymous, monotone and discriminating.

Note that this result generalises to any arbitrary number of superposed unimodal profiles. On the other hand it is not difficult to find superposed unimodal profiles at which the standard problems of Social Choice appear, such as for instance Condorcet cycles.

\section{Conclusion}

One of the main contribution of this chapter is in recognizing a common trait in all wellknown collective decision rules, that they all choose the mode as the outcome in case the underlying frequency distribution is unimodal. We also try to extend this result outside the domain of unimodal distribution. We present some of the interesting rules like Condorcet consistent rules, Borda rule, Plurality rule retain their property of choosing the mode even if the underlying distribution is $v$-tail-perturbed. As Borda rule and Plurality rule are "opposite extremes"in the class of score rules, we expect that a large subclass of score rules also choose the mode at such $v$-tail-perturbed unimodal distribution. We also expect that it is possible to predict more about the outcome of multimodal distributions resulting from superposed unimodal distributions. 


\section{Appendix-1}

This appendix is on some combinatorial results used in the proofs above. Throughout this appendix let $\bar{x}$ denote the top ordered candidate at $R^{*}$ and $y$ the worst ordered candidate at $R^{*}$. Let $\bar{R}^{*}=\left.R^{*}\right|_{A \backslash\{\bar{x}\}}$ and $\underline{R}^{*}=\left.R^{*}\right|_{A \backslash\{y\}}$. In several of the Lemmas below we use recursive formulae based on the cardinalities of $\overline{\mathbb{L}}^{k}=\{R: R$ is a linear order on $A \backslash\{\bar{x}\}$ with $\left.d\left(R, \bar{R}^{*}\right)=k\right\}, \overline{\mathbb{L}}_{x y}^{k}=\left\{R \in \overline{\mathbb{L}}^{k}:(x, y) \in R\right\}, \underline{\mathbb{L}}^{k}=\{R: R$ is a linear order on $A \backslash\{\underline{y}\}$ with $\left.d\left(R, \underline{R}^{*}\right)=k\right\}$ and $\underline{\mathbb{L}}_{x y}^{k}=\left\{R \in \underline{\mathbb{L}}^{k}:(x, y) \in R\right\}$, where $x$ and $y$ are different candidates such that in case of the first two sets $\bar{x} \neq x$ and in case of latter two sets $y \neq y$. Let these cardinalities be denoted by $\bar{l}(k), \bar{l}(k, x y), \underline{l}(k)$ and $\underline{l}(k, x y)$ respectively. Further, let $\bar{\delta}=\frac{1}{2}(m-1) \cdot(m-2)$ and $\bar{\rho}=\frac{1}{2} \bar{\delta}$ denote the diameter and radius at $m-1$ candidates.

Because the numbers $l(k), \bar{l}(k)$ and $\underline{l}(k)$ do not depend on the actual naming of the candidates we have that $\underline{l}(k)=\bar{l}(k)$. As the position of the interval $[x, y]_{R^{*}}=\left\{z \in A:(x, z) \in R^{*}\right.$ and $\left.(z, y) \in R^{*}\right\}$ differs in $\bar{R}^{*}$ from that in $\underline{R}^{*}$ some care has to be taken with the numbers $\underline{l}(k, x y)$ and $\bar{l}(k, x y)$. We argue at a later stage that these are equal as well. First we prove the following recursive relations for $l(k)$.

Lemma 4.4. For a distance $k$ from $R^{*}$

$$
\begin{aligned}
l(k) & =\sum_{t=0}^{\min \{k, m-1\}} \bar{l}(k-t) \\
& =\sum_{t=0}^{\min \{k, m-1\}} \underline{l}(k-t)
\end{aligned}
$$

Proof. Set $\mathbb{L}^{k}$ consists of those linear orders at distance $k-t$ from $\bar{R}^{*}$, where $\bar{x}$ has been swapped $t$ positions downwards. As $\bar{x}$ can at most move over $m-1$ positions the recursive formula follows evidently. The second equality follows similarly by deleting the worst ordered candidate but also because obviously $\bar{l}(k-t)=\underline{l}(k-t)$.

Having this recursive relation on $l(k)$ we can now prove that $l(k)$ strictly increases in $k$ that is for $k$ smaller than or equal to radius distance $\rho$. This completes the proof of Theorem 4.4 .

Lemma 4.5. $l\left(k_{1}\right)<l\left(k_{2}\right)$ for all $k_{1}<k_{2} \leqslant \rho$. 
Proof. It is obvious that $l(0)=\bar{l}(0)=1$. To prove the lemma, it is sufficient to prove it for the special case that $k_{1}=k-1$ and $k_{2}=k$, where $k \geqslant 1$. Hence we prove that $l(k)-l(k-1)>0$ for all $1 \leqslant k \leqslant \rho$. By the recursive formula of Lemma 4.4 we have, that if $k \leqslant m-1$, then

$$
l(k)-l(k-1)=\bar{l}(k),
$$

else if $k>m-1$, then

$$
l(k)-l(k-1)=\bar{l}(k)-\bar{l}(k-m),
$$

Therefore in case $k \leqslant m-1$, from Lemma 4.4 it is obvious that $l(k)-l(k-1)$ is positive. Now consider the case $k>m-1$. As by assumption $\rho \geqslant k$ we have $\frac{1}{4} m(m-1) \geqslant k$. So, $k-m \leqslant \frac{1}{4} m(m-1)-m=\frac{1}{4}\left(m^{2}-m-4 m\right)<\frac{1}{4}\left(m^{2}-3 m+2\right)=\frac{1}{4}(m-1)(m-2)=\bar{\rho}$. Therefore it is sufficient to prove that $\bar{\rho}$ is closer to $k$ than $k-m$, i.e., $k-\bar{\rho}<\bar{\rho}-k+m$. This is equivalent to $2 k<\bar{\delta}+m$. As $\bar{\delta}+m=\frac{1}{2}(m-1)(m-2)+m=\frac{1}{2}\left(m^{2}-3 m+2+\right.$ $2 m)=\frac{1}{2} m(m-1)+1$ the inequality $2 k<\bar{\delta}+m$ is equivalent to $k \leq \rho$. This proves the Lemma.

The following remarks are used later on.

Remark 4.5. (a) From the proof above we have that $|k-\bar{\rho}|<|k-m+1-\bar{\rho}|$ for all $k \leq \rho$.

(b) Noting that $d\left(R, R^{*}\right)=d\left(-R,-R^{*}\right)$ and $d\left(R^{*}, R\right)+d\left(R,-R^{*}\right)=d\left(R^{*},-R\right)+$ $d\left(-R,-R^{*}\right)=\delta$ for arbitrary linear orders $R$, we have that $l(k)=l(\delta-k)$ as well as $l(k, x y)=l(\delta-k, y x)$ for all distances $k$ and different candidates $x$ and $y$.

(c) In particular $(b)$ implies $l(k, x y)=l(\delta-k, y x)$ if $k=\rho$.

We now deduce recursive formula's for $l(k, x y)$.

Lemma 4.6. Let $x$ and $y$ be candidates.

(a) If $x \neq \bar{x}$, then $l(k, x y)=\sum_{t=0}^{\min \{k, m-1\}} \bar{l}(k-t, x y)$.

(b) If $y \neq \underline{y}$, then $l(k, x y)=\sum_{t=0}^{\min \{k, m-1\}} \underline{l}(k-t, x y)$. 
Proof. (Proof of (a)) Let $x \neq \bar{x}$. Now every linear orders in $\mathbb{L}_{x y}^{k}$ can be thought of as obtained from a linear order at distance $k-t$ from $\bar{R}^{*}$ preferring $x$ strictly to $y$ and then shifting $\bar{x}$ over $t$ position down. By this the formula follows.

(Proof of (b)) Is similar.

Next we show that $l(k, x y)=l(k, a b)$ when cardinalities of the intervals $[x, y]_{R^{*}}$ and $[a, b]_{R^{*}}$ are equal.

Lemma 4.7. Let $(x, y),(a, b) \in R^{*}$, such that the cardinalities of $[x, y]_{R^{*}}$ and $[a, b]_{R^{*}}$ are equal, say $\kappa$. Then $l(k, x y)=l(k, a b)$.

Proof. The proof is by induction on $m \geqslant 3$. The basis $m=3$ is straightforward although a bit cumbersome therefore it is left to the reader. So, here we only deduce the induction step and assume that $m \geqslant 4$.

In case the cardinalities of $[x, y]_{R^{*}}$ and $[a, b]_{R^{*}}$ are $m$, then $x=a$ and $y=b$. So, in this case the equality follows evidently. Therefore suppose that $\kappa<m$. Without loss of generality assume that $(x, a) \in R^{*}$ and $(a, x) \notin R^{*}$.

In case $\kappa=m-1$ we have that $x=\bar{x}, b=\underline{y}$ and $l(k, x y)=\sum_{t=0}^{\min \{k, m-1\}} \bar{l}(k-t, x y)$ and $l(k, a b)=\sum_{t=0}^{\min \{k, m-1\}} \underline{l}(k-t, a b)$. Now $x$ is ordered best at $\underline{R}^{*}$ and $y$ is ordered worst at this order. Where $a$ is ordered best at $\bar{R}^{*}$ and $b$ is ordered worst at that order. Because the cardinalities of $\mathbb{L}^{k}$ and $\mathbb{L}_{x y}^{k}$ do not depend on the actual names of the candidates involved, we have $\bar{l}(k-t, x y)=\underline{l}(k-t, a b)$ for appropriate $k$ and $t$. So, $l(k, x y)=l(k, a b)$.

In case $\kappa \leqslant m-2$ we can find $(c, d) \in R^{*}$ with the cardinalities of $[c, d]_{R^{*}}$ is equal to $\kappa$, $c \neq \bar{x}$ and $d \neq \underline{y}$. Applying Lemma 4.6 yields that $l(k, c d)=\sum_{t=0}^{\min \{k, m-1\}} \bar{l}(k-t, c d)=$ $\sum_{t=0}^{\min \{k, m-1\}} \underline{l}(k-t, c d), l(k, x y)=\sum_{t=0}^{\min \{k, m-1\}} \underline{l}(k-t, x y)$ and $l(k, a b)=\sum_{t=0}^{\min \{k, m-1\}} \bar{l}(k-$ $t, a b)$. By the induction hypothesis we have that $\underline{l}(k-t, c d)=\underline{l}(k-t, x y)$ and $\bar{l}(k-t, c d)=$ $\bar{l}(k-t, a b)$ for appropriate $k$ and $t$. So, $l(k, x y)=l(k, c d)=l(k, a b)$.

By Lemma 4.7 we are able to argue that $\underline{l}(k, x y)=\bar{l}(k, x y)$ as follows.

Lemma 4.8. For distance numbers $k$ we have $\underline{l}(k, x y)=\bar{l}(k, x y)$.

Proof. Note that the cardinalities of $\mathbb{L}^{k}$ and $\mathbb{L}_{x y}^{k}$ do not depend on the actual names of the candidates. So, as shifting the interval $[x, y]_{\underline{R}^{*}}$ one position up and renaming the alterna- 
tives yields the interval $[x, y]_{R^{*}}$. Lemma 4.7 implies that the shifting of the interval has no effect on the number $\underline{l}(k, x y)$ and renaming also has no effect. So, $\underline{l}(k, x y)=\bar{l}(k, x y)$.

To complete the proof of Theorem 4.2 we prove the following.

Lemma 4.9. Let distance $k \leq \rho$ and let $x$ and $y$ be different candidates with $(x, y) \in R^{*}$. Then

$$
l(k, x y) \geqslant l(k, y x) .
$$

Proof. The proof is by induction on the number of candidates. The basis $m=3$ is straight forward but cumbersome. We leave it to the reader. The induction step where $m \geq 4$ is proved by two cases. In the first case either $x$ is not ordered best at $R^{*}$ or $y$ is not ordered worst at $R^{*}$. Without loss of generality assume that $z$ is ordered worst at $R^{*}$, with $z \neq y$. So, by Lemma 4.6

$$
l(k, x y)=\sum_{t=0}^{\min \{k, m-1\}} \bar{l}(k-t, x y) .
$$

As $l(k)=\sum_{t=0}^{\min \{k, m-1\}} \bar{l}(k-t)$ and $\bar{l}(s)=\bar{l}(s, x y)+\bar{l}(s, y x)$ for all distances $s$, the induction argument yields that $\bar{l}(s, x y) \geqslant \frac{1}{2} \bar{l}(s)$ for all $s<\bar{\rho}$. Therefore we have $l(k, x y)$ $\geqslant \frac{1}{2} l(k)$. Hence, the desired result follows in case $k<\bar{\rho}$. For the case that $k \geqslant \bar{\rho}$ we can find a number $u$ such that $k=u+\lfloor\bar{\rho}\rfloor$. For $0 \leq s \leq u$ by Remark 4.5 (b) we have $\bar{l}(s+\lfloor\bar{\rho}\rfloor)=\bar{l}(\lceil\bar{\rho}\rceil-s)$ and $\bar{l}(s+\lfloor\bar{\rho}\rfloor, x y)=\bar{l}(\lceil\bar{\rho}\rceil-s, y x)$. This means that $\sum_{t=\lceil\bar{\rho}\rceil-u}^{k} \bar{l}(t, x y)=\frac{1}{2} \sum_{t=\lfloor\bar{\rho}\rfloor+1}^{k} \bar{l}(t)$. Straightforwardly $k<\rho$ and $m \geq 4$ imply $k<\bar{\delta}$. Now Remark 4.5 (a) and $k<\bar{\delta}$ imply $\lceil\bar{\rho}\rceil-u>\max \{k-m+1,0\}$. Therefore the induction argument yields that $l(k, x y) \geqslant \frac{1}{2} l(k)$ which proves this case.

Next consider the opposite case that is at $R^{*}$ candidate $x$ is ordered best and candidate $y$ is ordered worst. Suppose candidate $z$ is at position ${ }^{\mathbb{}} t$ at $R^{*}$. We can now think of that an ordering in $\mathbb{L}_{x y}^{k}$, with top $z$, is obtained from an ordering at distance $k-t$ from $\left.R^{*}\right|_{A \backslash\{z\}}$, where $z$ has to move $t$ positions to the top. Clearly $t \leq m-2$ as otherwise this operation would result in an order at which $y$ is above $x$. If $z=x$, then this results in $\bar{l}(k)$ orderings. If $z$ is at position 2 , this results in $\widetilde{l}(k-1, x y)$ orders. If $z$ is at position 3 in $R^{*}$

\footnotetext{
${ }^{8}$ There are $t-1$ candidates ordered above $z$ at $R^{*}$
} 
there are $\widetilde{l}(k-2, x y)$ orderings and so on, where $\widetilde{l}(k-t, x y)$ is the number of linear orders at distance $k-t$ from $\left.R^{*}\right|_{A \backslash\{z\}}$. Note that because these numbers are independent of the actual candidate deleted, which means $\widetilde{l}($.$) is the same as \bar{l}($.$) .$

$$
l(k, x y)=\bar{l}(k)+\sum_{t=1}^{\min \{k, m-2\}} \bar{l}(k-t, x y) .
$$

It is sufficient to prove that $2 \cdot l(k, x y)-l(k) \geqslant 0$. This means that we have to prove that $2 \cdot \bar{l}(k)+2 \cdot \sum_{t=1}^{\min \{k, m-2\}} \bar{l}(k-t, x y)-\sum_{t=0}^{\min \{k, m-1\}} \bar{l}(k-t) \geqslant 0$.

With the induction argument we have that $2 \cdot \sum_{t=1}^{\min \{k, m-2\}} \bar{l}(k-t, x y)-\sum_{t=1}^{\min \{k, m-2\}} \bar{l}(k-$ $t) \geqslant 0$. So, it remains to prove that $2 \cdot \bar{l}(k)-\bar{l}(k)-\bar{l}(k-m+1, x y) \geqslant 0$ or equivalently that $\bar{l}(k) \geqslant \bar{l}(k-m+1, x y)$. As by Remark 4.5 (a) $k$ is closer to $\bar{\rho}$ than $k-m+1$ we have by Lemma 4.5 and in case $k \geqslant \bar{\rho}$ using Remark 4.5 (b) that $\bar{l}(k)>\bar{l}(k-m+1) \geqslant$ $\bar{l}(k-m+1, x y)$ which completes the proof.

The following Lemma completes the proof of Theorem 4.3.

Lemma 4.10. Let distance $k \leq \rho$ and let $x$ and $y$ be different candidates with $(x, y) \in R^{*}$. Then

$$
\sum_{z \in A} l(k, x z)-l(k, y z) \geqslant 0
$$

Proof. It is sufficient to proof this inequality for the case that $x$ and $y$ are consecutively ordered at $R^{*}$, that is there are no candidate $z$, different from $x$ and $y$, which are ordered between $x$ and $y$ at $R^{*}$. In view of Lemma 4.7 we have that

$$
\sum_{z \in A} l(k, x z)-\sum_{z \in A} l(k, y z)=l(k, x \underline{y})-l(k, y \bar{x}) .
$$

Now as $(x, \underline{y})$ and $(\bar{x}, y)$ are in $R^{*}$ it follows that by Lemma 4.9 that $l(k, x \underline{y}) \geqslant \frac{1}{2} l(k) \geqslant$ $l(k, y \bar{x})$. This implies the desired result.

Let $x$ and $y$ be different candidates such that $(x, y) \in R^{*}$. It is sufficient to prove $p l(x)-$ $p l(y)>0$. Let $r_{x}$ and $r_{y}$ denote the rank of $x$ and $y$ in $R^{*}$ respectively. Let $u_{x}=m-r_{x}$ and 
$u_{y}=m-r_{y}$. Take $\mu_{x y}$ the average of $\bar{\rho}+u_{x}$ and $\bar{\rho}+u_{y}$, so $\mu_{x y}=\bar{\rho}+\frac{1}{2}\left(u_{x}+u_{y}\right)$, where $\bar{\rho}$ is the radius distance of the set of linear orders on $m-1$ candidates. Let $\bar{l}(k)$ be zero if $k$ is not in the interval $[0, \bar{\delta}]$. We need the following lemma to complete the proof of Theorem 4.4.

Lemma 4.11. $\bar{l}\left(k-u_{y}\right)<\bar{l}\left(k-u_{x}\right)$ for all $k$ such that $u_{x} \leqslant k<\mu_{x y}$.

Proof. By assumption we have that $k-u_{y}<k-u_{x}$. From $u_{x} \leqslant k$ we have $k-u_{x} \geqslant 0$. From $k<\mu_{x y}$, we have that

$$
\begin{aligned}
& k<\bar{\rho}+\frac{u_{x}+u_{y}}{2} \\
& \Longrightarrow 2 k-\left(u_{x}+u_{y}\right)<\bar{\delta} \\
& \Longrightarrow\left(k-u_{x}\right)-\bar{\rho}<\bar{\rho}-\left(k-u_{y}\right)
\end{aligned}
$$

If $k-u_{x} \leqslant \bar{\rho}$, then the above inequality implies $k-u_{y}<k-u_{x} \leqslant \bar{\rho}$. From Lemma 4.5, it is obvious that $\bar{l}\left(k-u_{y}\right)<\bar{l}\left(k-u_{x}\right)$. Else if $k-u_{y} \leqslant \bar{\rho}<k-u_{x}$, then the above inequality implies that $\bar{\rho}$ is closer to $k-u_{x}$ than it is to $k-u_{y}$. Applying Lemma 4.5 and Remark 4.5, in this case also we have that $\bar{l}\left(k-u_{y}\right)<\bar{l}\left(k-u_{x}\right)$. This completes the proof.

\section{Appendix-2}

One fundamental concept of social choice theory is the idea of a cycle, which may occur while aggregating individual preferences. It is usually called a Condorcet cycle [see Condorcet, 1785]. We use this notion several times in Chapter 4 and also throughout the thesis. So, let us recapitulate the idea with the following example.

Example 4.13. Suppose we have a voting situation with 3 candidates $a, b, c$ and 3 voters. Voters ranking over the candidates are displayed in the following Table 4.4. Here $p$ denotes the preference combination of the voters. Based on $p$, we have a pairwise comparison matrix also displayed in Table 4.4. The $(i, j)^{t h}$ cell of the pairwise comparison matrix denotes the number of voters who ranks candidate $i$ above candidate $j$, for all $i, j \in\{a, b, c\}$. Now, if it happens that entry in the $(i, j)^{t h}$ cell is larger than the entry in the $(j, i)^{t h}$ cell, then we say that candidate $i$ defeats candidate $j$ in majority. In case of equality we say they are socially indifferent. It is clear from Table 4.4 that, candidate $a$ defeats candidate $b$, 
candidate $b$ defeats candidate $c$ and candidate $c$ defeats candidate $a$ (all in majority). Now, transitivity of a relation $R$ means that, if $a R b$ and $b R c$, then $a R c$. But here in this example, if we combine the pairwise majority relations then they do not satisfy transitivity. This situation is known as a Condorcet cycle.

\begin{tabular}{|lll|}
\hline \multicolumn{3}{|c|}{$p$} \\
\hline$a$ & $b$ & $c$ \\
$b$ & $c$ & $a$ \\
$c$ & $a$ & $b$ \\
\hline 1 & 1 & 1 \\
\hline
\end{tabular}

\begin{tabular}{|c|c|c|c|}
\hline Candidate & $a$ & $b$ & $c$ \\
\hline$a$ & - & 2 & 1 \\
\hline$b$ & 1 & - & 2 \\
\hline$c$ & 2 & 1 & - \\
\hline
\end{tabular}

Table 4.4: Occurrence of a Cycle

$(a, b):(b, c):(c, a):$ Cycle

Let us see an example where a Condorcet cycle does not occur. Consider the preference profile and the respective pairwise comparison matrix displayed in Table 4.5. It is evident that, candidate $a$ defeats candidate $b$, candidate $a$ defeats candidate $c$ and candidate $c$ defeats candidate $b$ (all in majority). Therefore here we do not have any conflict with transitivity.

\begin{tabular}{|c|c|c|}
\hline \multicolumn{3}{|c|}{$\mathbf{q}$} \\
\hline & $a$ & $c$ \\
\hline & $c$ & $a$ \\
\hline$c$ & $b$ & $b$ \\
\hline 1 & 1 & 1 \\
\hline
\end{tabular}

\begin{tabular}{|c|c|c|c|}
\hline Candidate & $a$ & $b$ & $c$ \\
\hline$a$ & - & 3 & 2 \\
\hline$b$ & 0 & - & 1 \\
\hline$c$ & 1 & 2 & - \\
\hline
\end{tabular}

Table 4.5: Non-occurrence of a Cycle

$(a, b):(a, c):(c, b) \Longrightarrow a c b$ 



\section{Bibliography}

Arrow, K. J., A Difficulty in the Concept of Social Welfare, The Journal of Political Economy, 58(4), 328-346, 1950

Black, D., On the Rationale of Group Decision Making, The Journal of Political Economy, $56,23-34,1948$

Black D., The Theory of Committees and Elections, Cambridge, 1958

Border K. C., Jordan J. S., Straightforward Elections, Unanimity and Phantom Voters, The Review of Economic Studies 50, 153 - 170, 1983

Condorcet, M. J. A. N., Caritat (marquis de)(1785) Essai sur lapplication de lanalyse à la probabilité des décisions rendues à la pluralité des voix, 1785

Chatterjee S., Peters H., and Storcken T., Locating a public good on a sphere, Economics Letters, 139, 4648, 2016

Chatterjee S., Storcken T., Frequency based analysis of voting rules, GSBE Research Memoranda, No. 006, 2017

Ehlers L., Independence axioms for the provision of multiple public goods as options, Mathematical Social Sciences, 41, 239-250, 2001 
Ehlers L., Multiple public goods and lexicographic preferences: replacement principle, Journal of Mathematical Economics, 37, 1- 15, 2002

Fishburn P., Gehrlein W., An analysis of voting procedures with nonranked voting, Behav Sci 22, 178-185, 1977

Gehrlein W., Condorcet winners in dual cultures, Presented at National Meeting of PublicChoice Society, New Orleans, LA, 1978

Gehrlein, W., Condorcet's Paradox, Springer, Berlin, 2006

Gibbard A., Manipulation of voting schemes: a general result, Econometrica: journal of the Econometric Society, 41, 587-601, 1973

Gillett R., Conflict and indecisiveness in collective decision procedures, Presented at Mathematical and Statistical Section of the British Psychological Society, Birmingham, UK, 1976

Gillett R., A recursion relation for the probability of the paradox of voting, Journal of Economic Theory 18, 318-327, 1978

Gordon S., Solidarity in choosing a location on a cycle, Soc Choice Welfare, 29, 125-147, 2007

Grofman B., Regenwetter M., and Tsetlin, I., The impartial culture maximizes the probability of majority cycles, Social Choice and Welfare, 21(3), 387-398, 2003

Grofman B., Marley A.A.J., Regenwetter, M., and Tsetlin I., Behavioral Social Choice: Probabilistic Models, Statistical Inference, and Applications, Cambridge:Cambridge University Press, 2006

Hansson, B., The existence of group preference functions, Public Choice, 28, 1976

Heo E. J., Strategy-proof rules for two public goods: double median rules, Social Choice and Welfare, 41, 895-922,2013

Kim K. H., Roush F. W., Properties of Consistent Voting Systems, International Journal of Game Theory, 10, 45-52, 1981

Kirman, A. P., Sondermann, D., Arrow's theorem, many agents, and invisible dictators, 5(2), 267-277, 1972 
Maskin, E. S., MECHANISM DESIGN: HOW TO IMPLEMENT SOCIAL GOALS, Nobel Prize Lecture, December 8, 2007

Merlin, V., Tataru M., Valognes F., On the probability that all decision rules select the same winner, Journal of Mathematical Economics, 33, 183-207, 2000

Miyagawa E., Locating libraries on a street, Social Choice and Welfare, 18, 527-541, 2001

Moulin H., On Strategy-Proofness and Single Peakedness, Public Choice, 35, 437- 455, 1980

Öztürk M., Strategy-proofness and single-dipped preferences, PhD Thesis, Maastricht University, 2013

Öztürk M., Peters H., and Storcken T., Strategy-proof location of a public bad on a disc, Economics Letters, 119, 14 - 16,2013

Öztürk M., Peters H., and Storcken T., On the location of public bads: strategy-proofness under two-dimensional single-dipped preferences, Economic Theory, 56, 83 - 108, 2014

Peters, H., Stel, H., and Storcken, T., Pareto Optimality, Anonymity, and StrategyProofness in Location Problems, International Journal of Game Theory, 21, 221235,1992

Riker, W., Liberalism against Populism: A Confrontation between the Theory of Democracy and the Theory of Social Choice, San Francisco: W. H. Freeman \& Co., 1982

Satterthwaite M. A., Strategy-proofness and Arrow's conditions: Existence and correspondence theorems for voting procedures and social welfare functions, Journal of Economic Theory, 10(2), 187-217, 1975

Vickrey, W., Counterspeculation, auctions, and competitive sealed tenders, The Journal of finance, 16(1), 8 - 37, 1961

Weymark, J. A.,A unified approach to strategy-proofness for single-peaked preferences, SERIEs, 2(4), 529-550, 2011 



\section{Valorisation}

Valorisation of learning or innovation exchange and can be defined as:

The procedure of significant worth creation out of learning, by making this information reasonable and accessible for monetary or societal use and to make an interpretation of this into high-potential items, administrations, forms and mechanical action. Valorisation is not just about 'money', but rather about effect that can be made through the exchange of logical learning. Aside from commercialising your development through a turn off organization, patent, or permit, there are numerous approaches to have effect, for instance through the exchange of learning to human services associations, by making information and ability accessible for other (societal) associations and by conveying your insight to the overall population.

This thesis broadly studies how to aggregate preferences or opinions of people in a society. In the first part of this thesis we study the issue of selecting locations for public facilities within a region. In real life a social planner has to decide where to locate such public facilities taking into account the preferences of people in the concerned region. So, the planner must have a rule which he applies while deciding the location. This thesis studies which rules are possible to achieve the desired result. Furthermore to make this choice non manipulable by anyone in the society we impose a condition called strategy-proofness, which ensures that truthful reporting of preferences is weakly dominant strategy for each member in the region under consideration. We also put some additional properties which 
are particular to the specific location problem.

In the second chapter we study where to locate a public good(bad) on a sphere. Considering earth as a sphere this study is easily applicable in real life. Notice that the public good(bad) in this context has to be global in effect. Global public goods are products with benefits as well as costs that possibly reach out to all nations, individuals, and eras. Global public goods are in a double sense open: they are open rather than private; and they are worldwide instead of national. For instance, greenhouse gasses must not rise and weight the air to the degree they do. The majority of this is today a matter of policy choice. Another modern life example is to choose a location for a huge data center for keeping all human knowledge safe and secure. On the other hand global public bads are also required for mankind but they have negative externalities. For instance, to locate a nuclear experiment center which is much larger than the Large Hadron Collider. This might be required for the advancement of quantum physics but it could also be potentially risky to the neighbourhood. Interesting aspect of this chapter is that the result for public goods applies to for public bads.

We can apply the results from chapter three in a situation where for example, the municipality of Maastricht wants to locate two libraries. People in Maastricht are asked to report their preferences. This is also applicable if the municipality wants to locate two hospitals or two sports centres but not if they want one hospital and one sports centre. So, two public facilities must be identical. Here, we characterise a set of rules that only cares about the most preferred location for everyone in the region.

In part two chapter four we deal with voting problem that appears in a nation-wide election. As we know that a major election reform is happening in Italy as parliament approves new law. The main issue in Voting is to choose a voting method that satisfies many properties. But every voting method has some disadvantages. We tackle this situation in a different manner. We treat voters preferences as frequency distributions. Any such frequency distribution can be written as a so called multimodal frequency distribution. We study unimodal frequency distributions which are building blocks of multimodal distributions. We show that under a unimodal distribution candidate with highest frequency must be chosen by any existing voting rule. Aggregation of several unimodal distributions result in a multimodal distribution. It is possible to predict the outcome from a multimodal distribution by studying its unimodal components. Thus this study shows a possibility of having a general voting method which can be applied in reality. 


\section{Curriculum Vitae}

Swarnendu Chatterjee was born on December 24, 1990 in Kolkata, India. He studied Bachelor of Science (BSc) with Statistics major at Presidency College, University of Calcutta. In 2011, he received his BSc degree. He studied Master of Statistics(MStat) at Indian Statistical Institute, Delhi. During Master's he specialised in Quantitative Economics. In 2013, he received his MStat degree.

He has worked as a Business Analyst at Hewlett and Packard, Bangalore till July, 2014. Then he joined Maastricht University as a $\mathrm{PhD}$ candidate under the supervision of Dr. Hans Peters and Dr. Ton Storcken. The outcomes of his research is presented in this thesis. Swarnendu presented his research at various international conferences. Some parts of this thesis have been accepted for publication in international refereed academic journals. 

Index

antipodal, 10, 13

azimuth, 19

Borda rule, 6, 54, 55, 61, 73-75, 79, 82

Borda score, 67, 73

box, 26

box property, 28

coalitional strategy-proofness, 4

Condorcet consistent rules, 54, 55, $61-63,68,70,72,74,82$

Condorcet cycle, 9,89

coordinatewise, 10, 20, 21

dip, 2

dominant strategy, 27

double generalised median rule, 39

double uncompromising, 28

Elliptical Indifference Curve, 30

frequency, 2, 5, 6, 53-55, 57, 58, 61, 65, $68,69,71,73,74,82$ impossibility, 4

Kemeny distance, 5, 56-58, 80

lexmax preference, 26

manipulable, 11

Maskin monotonic, 27

median, 9

multimodal, 6, 53-56, 80, 82

Nanson rule, 67

non-manipulability, 2

Option set, 28

pairwise comparison matrix, 88

Pareto optimality, 3

peak, 2

peaks only, 27

Plurality rule, 6, 54, 55, 75-79, 82

polar, 19

public good, 4

quadrants, 24 
single-dipped, 2

single-peaked, 2, 9

strategy-proof, 9, 27

strict strategy proofness, 4

tail-perturbed unimodal distribution, 69,
82

transitivity, 89

Uncompromisingness, 3

unimodal, 5, 6, 53-56, 58, 61, 64-70,

$72,74,75,79-82$ 